\title{
Astrophysical properties of star clusters in the Magellanic Clouds homogeneously estimated by ASteCA ${ }^{\star}$
}

\author{
G. I. Perren ${ }^{1,3}$, A. E. Piatti ${ }^{2,3}$, and R. A. Vázquez ${ }^{1,3}$ \\ 1 Facultad de Ciencias Astronómicas y Geofísicas (UNLP), IALP-CONICET, 1900 La Plata, Argentina \\ e-mail: gabrielperren@gmail.com \\ 2 Observatorio Astronómico, Universidad Nacional de Córdoba, 5000 Córdoba, Argentina \\ 3 Consejo Nacional de Investigaciones Científicas y Técnicas (CONICET), 1917 Buenos Aires, Argentina
}

Received 12 August 2016 / Accepted 20 January 2017

\begin{abstract}
Aims. We seek to produce a homogeneous catalog of astrophysical parameters of 239 resolved star clusters, located in the Small and Large Magellanic Clouds, observed in the Washington photometric system.

Methods. The cluster sample was processed with the recently introduced Automated Stellar Cluster Analysis (ASteCA) package, which ensures both an automatized and a fully reproducible treatment, together with a statistically based analysis of their fundamental parameters and associated uncertainties. The fundamental parameters determined for each cluster with this tool, via a color-magnitude diagram (CMD) analysis, are metallicity, age, reddening, distance modulus, and total mass.

Results. We generated a homogeneous catalog of structural and fundamental parameters for the studied cluster sample and performed a detailed internal error analysis along with a thorough comparison with values taken from 26 published articles. We studied the distribution of cluster fundamental parameters in both Clouds and obtained their age-metallicity relationships.

Conclusions. The ASteCA package can be applied to an unsupervised determination of fundamental cluster parameters, which is a task of increasing relevance as more data becomes available through upcoming surveys.
\end{abstract}

Key words. galaxies: fundamental parameters - galaxies: star clusters: general - Magellanic Clouds - methods: statistical catalogs - techniques: photometric

\section{Introduction}

Analyses of star clusters can serve as the foundation for the study of the structure, dynamics, star formation history, and chemical enrichment of a galaxy. Star clusters in the Magellanic Clouds (MCs) are made up of a varying number of coeval stars sharing a chemical composition, and are assumed to be located relatively at the same distance from the Sun and affected by roughly the same amount of reddening. These factors facilitate the estimation of their fundamental parameters and thus the properties of their host galaxy. New developments in astrophysical software enable the homogeneous processing of different types of databases of star clusters. The article series by Kharchenko et al. (see Kharchenko et al. 2005; Schmeja et al. 2014, and references therein) and the integrated photometry based derivation of age and mass for 920 clusters presented in Popescu et al. (2012), based on their MASSsive CLuster Evolution and ANalysis package (MASSCLEAN; Popescu \& Hanson 2009) ${ }^{1}$, are examples of semi-automated and automated packages applied on a large number of clusters.

However, there is no guarantee that by employing a homogeneous method, we will obtain similar parameter values from the same cluster photometric data set across different studies. This is particularly true when the methods require user

\footnotetext{
* A table with the estimated fundamental parameters for the 239 clusters analyzed is only available at the CDS via anonymous ftp to cdsarc.u-strasbg. fr $(130.79 .128 .5)$ or via http://cdsarc.u-strasbg.fr/viz-bin/qcat?]/A+A/602/A89

1 http://www.massclean.org/
}

intervention, which makes the results subjective to some degree. In Netopil et al. (2015) the open cluster parameters age, reddening, and distance are contrasted throughout seven published databases. All these databases were found to show nonnegligible offsets in their fundamental parameter values. This result highlights an important issue: most of the color-magnitude diagram (CMD) isochrone fits are carried out by eye, adjusting correlated parameters independently and often omitting a proper error treatment (see von Hippel et al. 2014, for a more detailed description of this problem). When statistical methods are employed, the used code is seldom publicly shared to allow scrutiny by the community. There is then no objective way to asses the underlying reliability of each set of results. Lacking this basic audit, the decision of which database values to use becomes a matter of preference.

As demonstrated by Hills et al. (2015), assigning precise fundamental parameters for an observed star cluster is not a straightforward task. Using combinations of up to eight filters $\left(U B V R I J H K_{\mathrm{s}}\right)$ and three stellar evolutionary models, these investigators analyzed the NGC 188 open cluster with a Bayesian isochrone fitting technique implemented in their BASE-9 package ${ }^{2}$, and arrived at statistically different results depending on the isochrones and filters used. NGC 188 is a $\sim 4$ Gyr cluster with a well-defined main sequence (MS) that is mostly unaffected by field star contamination and that has proper motions and radial velocities data available. A typical situation - in which a star cluster is observed through fewer

\footnotetext{
2 http://webfac.db.erau.edu/ vonhippt/base9/
} 
filters, affected by a non-negligible amount of field star contamination, and lacks information about its dynamics - is significantly more complicated to analyze. A mismatch between theoretical evolutionary models, along with an inability to reproduce clusters in the unevolved MS domain, had already been reported in Grocholski \& Sarajedini (2003).

The aforementioned difficulties in the analysis of star cluster CMDs will only increase if the study is carried out by eye, since a) the number of possible solutions manually fitted is several orders of magnitude smaller than that handled by a code; b) correlations between parameters are almost entirely disregarded; c) uncertainties cannot be assigned through valid mathematical means and are often not assigned at all; and d) the final values are necessarily highly subjective. In the last 30 years, many authors have applied some form of statistical analysis to derive the fundamental parameters of star clusters. We give in Sect. 2.9 of Perren et al. (2015, hereafter Paper I) a non-exhaustive list of articles in which these methods were employed. Still, by-eye studies continue to be used. This is because most statistical methods developed are either closed-source, or restricted to a particular form of analysis (or both). There is a clear need for an automated general method with a fully open and extensible code base that takes as much information into account as possible and is capable of generating reliable results.

In Paper I we presented the Automated STEllar Cluster Analysis (ASteCA) package, aimed at allowing an accurate and comprehensive study of star clusters. The code is released under a GPL v3 general public license ${ }^{3}$ and can be downloaded from its official site ${ }^{4}$. Through a mostly unassisted process the code analyses positional and photometric data sets of star clusters to derive their fundamental parameters and uncertainties. As shown in Paper I, the code is able to assign precise parameter values for clusters with low to medium field star contamination and gives reasonable estimations for heavily contaminated clusters. Every part of this astrophysical package is open and publicly available and its development is ongoing.

In the present work we apply ASteCA on 239 clusters in the Small and Large Magellanic Clouds (S/LMC), distributed up to $\sim 5^{\circ}$ and $\sim 8^{\circ}$ in angular distance from their centers, respectively. The MCs are located close enough to us to allow the study of their resolved star clusters. The large number of cataloged clusters, 4000 are listed in the Bica et al. (2008) catalog, makes them an invaluable resource for investigating the properties of the two most massive galaxies that orbit the Milky Way. The reddening that affects the MCs is relatively small, except for a few regions, such as 30 Doradus in the LMC, where $E_{B-V}$ can reach values above 0.4 mag (Piatti et al. 2015a). The overall low levels of reddening simplifies the research of the clusters in these two galaxies. We use photometric data sets in the $C T_{1}$ Washington system (Canterna 1976; Geisler 1996), known to be highly sensitive to metal abundance for star clusters older than $\sim 1 \mathrm{Gyr}$ (Geisler \& Sarajedini 1999). The results obtained here regarding the metal content are thus of relevance for the analysis of the MCs chemical enrichment history.

This is the first study in which such a large sample of resolved star clusters is homogeneously analyzed in an automatic way with their fundamental parameters statistically estimated rather than fitted by eye or fixed a priori. Being able to assign metal content for $100 \%$ of our sample is particularly important, especially compared to other star cluster catalogs. The latest version of the well-known DAML02 database (v3.5, 2016 Jan. 28;

\footnotetext{
3 https://www.gnu.org/copyleft/gpl.html

4 http://asteca.github.io
}

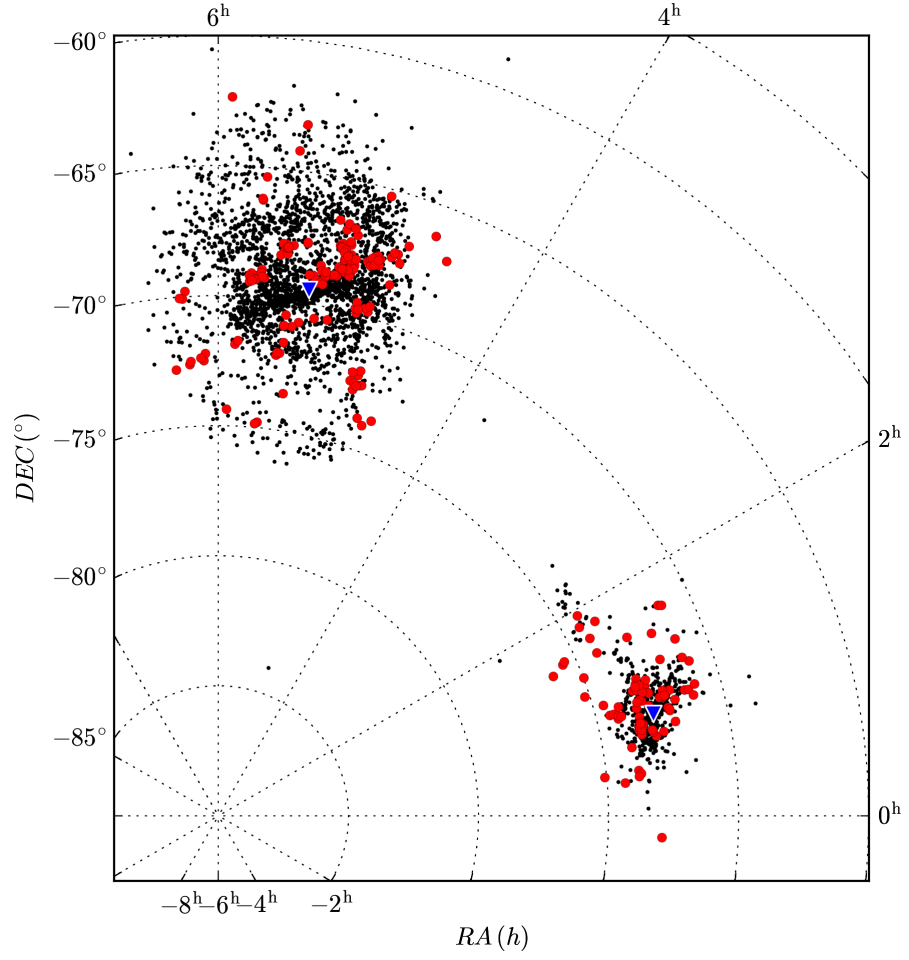

Fig. 1. Distribution of our set of analyzed clusters (red circles) superimposed on to the Bica et al. (2008) database of 3740 star clusters (black dots) for both MCs. The assumed centers for the Clouds are indicated with blue triangles.

Dias et al. 2002) $)^{5}$, for example, reports abundances for only $13 \%$ out of the 2167 clusters cataloged. Estimations for the total cluster mass is given in few cases, if integrated photometry is provided.

This article is structured as follows. In Sect. 2 we present the star cluster sample used in this work along with numerous studies used to compare and validate our results. Sections 3 and 4 describe the estimation of the fundamental parameters derived with ASteCA and analyze their uncertainties, respectively. In Sect. 5 a detailed comparison of our results with published values from the literature is performed. Section 6 shows the distribution of cluster fundamental parameters in our catalog and the age-metallicity relationships (AMRs) for the cluster system. Section 7 summarizes our results and concluding remarks.

\section{Clusters sample}

The data set used in this work consists of $C T_{1}$ Washington photometry for 239 star clusters: 150 located in the LMC and the rest in the SMC. These clusters were selected because they were readily available; are already analyzed in the literature, meaning we can compare the published parameters with the estimates produced in this work; and are sufficiently dispersed throughout both galaxies. In Fig. 1 we show their spatial distribution.

Table 1 lists the 19 articles that analyzed the same $C T_{1}$ photometry used by ASteCA in the current study. Hereafter, we refer to this group as the literature. Metallicities, ages, reddenings, and distance moduli $\left(\mu_{\circ}\right)$ were estimated or assigned in the literature, except for the 36 clusters in Piatti (2011b), which had only their ages estimated via the $\delta T_{1}$ index (Phelps et al. 1994; Geisler et al. 1997). In most of the literature metallicities

\footnotetext{
5 http://www.wilton. unifei . edu.br/ocdb/
} 
Table 1. Sources of the $C T_{1}$ data sets used in this work.

\begin{tabular}{lccc}
\hline \hline Article & $N$ & Galaxy & Telescope \\
\hline Geisler et al. (2003) & 8 & LMC & CTIO 0.9 m \\
Piatti et al. (2003b) & 5 & LMC & CTIO 0.9 m \\
Piatti et al. (2003a) & 6 & LMC & CTIO 0.9 m \\
Piatti et al. (2005) & 8 & SMC & CTIO 0.9 m \\
Piatti et al. (2007a) & 4 & SMC & CTIO 0.9 m \\
Piatti et al. (2007c) & 2 & SMC & Danish 1.54 m \\
Piatti et al. (2007b) & 2 & SMC & Danish 1.54 m \\
Piatti et al. (2008) & 6 & SMC & Danish 1.54 m \\
Piatti et al. (2009) & 5 & LMC & CTIO 0.9 m/ \\
& & & Danish 1.54 m \\
Piatti et al. (2011b) & 3 & LMC & CTIO 0.9 m \\
Piatti et al. (2011a) & 14 & SMC & CTIO 1.5 m \\
Piatti (2011a) & 9 & SMC & Blanco 4 m \\
Piatti (2011b) & 36 & LMC & Blanco 4 m \\
Piatti (2011c) & 11 & SMC & Blanco 4 m \\
Piatti (2012) & 26 & LMC & Blanco 4 m \\
Piatti \& Bica (2012) & 4 & SMC & Blanco 4 m \\
Palma et al. (2013) & 23 & LMC & Blanco 4 m \\
Maia et al. (2013) & 29 & SMC & Blanco 4 m \\
Choudhury et al. (2015) & 38 & LMC & Blanco 4 m \\
\hline
\end{tabular}

Notes. $N$ refers to the number of clusters analyzed per article.

Table 2. Sources of Johnson-Kron-Cousins photometric data sets for some clusters in our sample.

\begin{tabular}{lccc}
\hline \hline Article & $N$ & Galaxy & Phot \\
\hline Pietrzynski \& Udalski (1999, P99) & 7 & SMC & $B V I$ \\
Pietrzynski \& Udalski (2000, P00) & 25 & LMC & $B V I$ \\
Hunter et al. (2003, H03) & 62 & S/LMC & $U B V R$ \\
Rafelski \& Zaritsky (2005, R05) & 24 & SMC & $U B V I$ \\
Chiosi et al. (2006, C06) & 16 & SMC & $V I$ \\
Glatt et al. (2010, G10) & 61 & S/LMC & $U B V I$ \\
Popescu et al. (2012, P12) & 48 & LMC & $U B V R$ \\
\hline
\end{tabular}

Notes. $N$ refers to the number of clusters in common with our sample per article.

and the distance moduli are fixed to $[\mathrm{Fe} / \mathrm{H}]=-0.7$ dex, $[\mathrm{Fe} / \mathrm{H}]=-0.4 \mathrm{dex}$, and $\mu_{\circ}=18.9 \mathrm{mag}$ and $\mu_{\circ}=18.5 \mathrm{mag}$, for the S/LMC, respectively. Ages reported in the literature were obtained by eye, either through the standard isochrone fitting technique or applying the $\delta T_{1}$ index method. Reddenings were estimated in almost all cases interpolating the maps of either Burstein \& Heiles (1982), Schlegel et al. (1998), or Haschke et al. (2011). Maia et al. (2013) presented total mass estimations for their 29 star clusters sample.

Our cluster sample was also partially studied via JohnsonKron-Cousins photometry, as listed in Table 2. This group is referred to as the databases (DBs), as a way to distinguish them from the literature. In Sect. 5 we compare the parameter values obtained by ASteCA, to those given in both the literature and the databases.

\section{Estimation of star cluster parameters}

Fundamental parameters (metallicity, age, distance modulus, reddening, and mass) and structural parameters (center coordinates, radius, contamination index, approximate number of members, membership probabilities, and true cluster probability) were obtained either automatically or semi-automatically with ASteCA. A detailed description of the functions built within this tool can be found in Paper I and in the online documentation ${ }^{6}$ of this code. The resulting catalog can be accessed via VizieR. We have made available the Python codebase developed to analyze the data obtained with ASteCA and generate the figures in this article ${ }^{7}$. Output images generated by ASteCA for each cluster can be accessed through a separate public code repository ${ }^{8}$.

\subsection{Ranges for fitted fundamental parameters}

To process a cluster, the user must provide ASteCA with a suitable range of accessible values for each fundamental parameter by setting a minimum, a maximum, and a step. As explained in Sect. 3.4, each combination of values from the five fundamental parameters represents a unique synthetic CMD, or model. The larger the number of accessible parameter values, the larger the amount of models the code processes to find the synthetic CMD that best matches the observed cluster CMD. Ranges and steps were selected to provide a balance between a large interval and a computationally manageable number of total models; see Table 3. Special care was taken to avoid defining ranges that could bias the results toward a particular region of any fitted parameter.

Unlike most previous works where the metallicity is a fixed value, we do not make assumptions on the metal content of the cluster. Our $[\mathrm{Fe} / \mathrm{H}]$ interval completely covers the usual metallicities reported for MC clusters. The age range encompasses almost the entire allowed range of the CMD service ${ }^{9}$ from which the theoretical isochrones were obtained (see Sect. 3.4).

The maximum allowed value for the reddening of each cluster was determined through the Magellanic Clouds extinction values (MCEV) reddening maps (Haschke et al. 2011) ${ }^{10}$, while the minimum value is always zero. We used TOPCAT ${ }^{11}$ to query $E_{V-I}$ values from these maps, within a region as small as possible around the position of each cluster. For $85 \%$ of our sample we found several regions with associated reddening values within a box of $0.5 \mathrm{deg}$ centered on the position of the cluster. For the remaining clusters, larger boxes had to be used. The two most extreme cases are NGC $1997\left(\alpha=5^{\mathrm{h}} 30^{\mathrm{m}} 34^{\mathrm{s}}\right.$, $\left.\delta=-63^{\circ} 12^{\prime} 12^{\prime \prime}[\mathrm{J} 2000.0]\right)$ and OHSC28 $\left(\alpha=5^{\mathrm{h}} 55^{\mathrm{m}} 35^{\mathrm{s}}, \delta=\right.$ $-62^{\circ} 20^{\prime} 43^{\prime \prime}$ [J2000.0]) in the outers of the LMC, where boxes of $4 \mathrm{deg}$ and $6 \mathrm{deg}$, respectively, where needed to find a region with assigned reddening values. In both cases, the reddenings given by the Schlafly \& Finkbeiner (2011) map for their coordinates are up to two times smaller than those found in the MCEV map. We adopted the largest $E_{V-I}$ value of each region, $\mathrm{MCEV}_{\max }$, as the upper limit in the reddening range. Three steps are used to ensure that the reddening range is partitioned similarly for all $\mathrm{MCEV}_{\max }$ values: 0.01 for $\mathrm{MCEV}_{\max }>0.1,0.02$ if $0.05 \leq$ $\mathrm{MCEV}_{\max } \leq 0.1$, and 0.005 for $\mathrm{MCEV}_{\max }<0.05$. The $E_{V-I}$ extinction is converted to $E_{B-V}$ following the Tammann et al. (2003) relation, $E_{V-I}=1.38 E_{B-V}$. An extinction law of $R_{v}=3.1$ is applied throughout the analysis.

Mean distance moduli for the S/LMCs were taken from de Grijs \& Bono (2015) and de Grijs et al. (2014). Lineof-sight (LOS) depths for the MCs (front to back, $\pm 1 \sigma$ ) have been reported to span up to $20 \mathrm{kpc}$ in their deepest

\footnotetext{
6 http://asteca.rtfd.org

https://github.com/Gabriel-p/mc-catalog

8 https://github.com/Gabriel-p/mc-catalog-figs

9 http://stev.oapd.inaf.it/cgi-bin/cmd

10 http://dc.zah. uni-heidelberg.de/mcextinct/q/cone/ form

11 http://www.star.bris.ac.uk/ mbt/topcat/
} 
Table 3. Fundamental ranges of parameters used by ASteCA on our set of 239 clusters.

\begin{tabular}{lcccc}
\hline \hline Parameter & Min & Max & Step & $N$ \\
\hline$[\mathrm{Fe} / \mathrm{H}]$ & $\sim-2.2$ & 0 & $\sim 0.1$ & 23 \\
log $($ age $/ \mathrm{yr})$ & 6. & 10.1 & 0.05 & 82 \\
$E_{B-V}$ & 0.0 & $\mathrm{MCEV}_{\max }$ & $\sim 0.01$ & $\sim 12$ \\
$\mu_{\mathrm{SMC}}$ & 18.86 & 19.06 & 0.02 & 10 \\
$\mu_{\mathrm{LMC}}$ & 18.4 & 18.6 & 0.02 & 10 \\
Mass $\left(M_{\odot}\right)$ & 10 & {$[1,3] \times 10^{4}$} & 200 & {$[50,150]$} \\
\hline
\end{tabular}

Notes. The approximate number of values used for each parameter is $N$. This gives a combined total of $\sim 2.3 \times 10^{7}$ possible models (or synthetic cluster CMDs), which could be theoretically matched to each studied cluster in our sample.

regions (Subramanian \& Subramaniam 2009; Nidever et al. 2013; Scowcroft et al. 2016). The $0.1 \mathrm{mag}$ deviations allowed in this work give LOS depths of $\sim 5.7 \mathrm{kpc}$ and $\sim 4.6 \mathrm{kpc}$ for the S/LMC. This covers more than half of the average LOS depths found in Subramanian \& Subramaniam (2009) for the SMC (bar: $4.9 \pm 1.2 \mathrm{kpc}$, disk: $4.23 \pm 1.48 \mathrm{kpc}$ ), and the LMC (bar: $4.0 \pm 1.4 \mathrm{kpc}$, disk: $3.44 \pm 1.16 \mathrm{kpc}$ ). Although this is not enough to cover the entire observed depth ranges, it gives the distance moduli liberty to move around their mean values when all parameters are adjusted.

The maximum total cluster mass was set to $10000 M_{\odot}$. To estimate this value, a first rough pass was performed with ASteCA for all clusters in our data set. Most of the clusters were assigned total masses below $5000 M_{\odot}$, making the $10000 M_{\odot}$ limit a reasonable value. This was true for all except 15 visibly massive clusters, for which the maximum mass was increased to $30000 M_{\odot}$. We see in Sect. 5.2.1 that the code is systematically underestimating masses because of the stellar crowding effect on our set of Washington photometry.

The binary fraction was fixed to 0.5 , which is considered a reasonable estimate for clusters (von Hippel 2005; Sollima et al. 2010), to avoid introducing an extra degree of complexity into the fitting process. Secondary masses are randomly drawn from a uniform mass ratio distribution of the form $0.7 \leq q \leq 1$, where $q=M_{2} / M_{1}$, and $M_{1}, M_{2}$ are the primary and secondary masses. This range for the secondary masses was found for the LMC cluster, NGC 1818, in Elson et al. (1998) and represents a value commonly used in analysis regarding the MCs (see Rubele et al. 2011, and references therein).

\subsection{Center and radius assignment}

ASteCA employs a two-dimensional Gaussian kernel density estimator (KDE) to determine the center of the cluster. A radial density profile (RDP) is used to estimate the radius of the cluster, as the point where the RDP reaches the mean density of the surrounding field.

The density of cluster members must make it stand out over the combination of foreground/background stars with no other overdensity present in the observed frame (this limitation is planned to be lifted in upcoming versions of ASteCA). A portion of the surrounding field should be visible and the RDP must be reasonably smooth. When these conditions are not met, the semi-automatic mode can be used. Here center coordinates are either obtained based on an initial set of approximate values or manually fixed along with the radius value.
For $\sim 66 \%$ of our sample, center coordinates were obtained via a KDE analysis based on initial approximate values. Radii were calculated for $\sim 60 \%$ of the clusters through an RDP analysis of their surrounding fields. The remaining clusters are those that are highly contaminated, contain very few observed stars, and/or occupy most of the observed frames. This means that an estimation of their centers and/or radii was not possible and their values were manually fixed.

The contamination index $(C I)$ is a parameter related to the number of foreground/background stars in the cluster region. See Paper I, Sect. 2.3.2 for a complete mathematical definition of the index. Values of $0,0.5$, and $>0.5$ mean that the cluster is not contaminated by field stars, an equal number of field stars and cluster members are present in the cluster region, and more field stars are expected within the cluster region than cluster stars, respectively. For reference, the average $C I$ for the set of clusters with manual radii assignment is $C I \simeq 0.9 \pm 0.2$, and $C I \simeq 0.6 \pm$ 0.2 for those clusters whose radii were estimated in automatic mode.

\subsection{Field star decontamination}

A decontamination algorithm (DA) was employed on the $C T_{1}$ CMD of each processed cluster to remove field star contamination. The Bayesian DA presented in Paper I was improved for the present analysis. The new DA works in two steps: first, the original Bayesian membership probability (MP) assignation is applied to the CMD of all stars within the cluster region (see $\mathrm{Pa}$ per I for more details). After that the CMD is binned into cells and a cleaning algorithm is used to remove stars of low MPs cell by cell, as shown in Fig. 2. By default ASteCA uses the Bayesian blocks method ${ }^{12}$ introduced in Scargle et al. (2013) via the implementation of the astroML package (Vanderplas et al. 2012). While Bayesian blocks binning is the default setting in ASteCA, several other techniques for CMD star removal are available as well as five more binning methods. This second step is similar to the DA developed in Bonatto \& Bica (2007, B07), which uses a simpler rectangular grid. The main difference, aside from the binning method employed, is that ASteCA removes stars based on their MPs rather than removing the stars randomly as in B07.

Approximately $70 \%$ of our sample was processed with these default settings. The remaining $\sim 30 \%$, those with a low number of cluster stars or those that were heavily contaminated, were processed with modified settings to allow a proper field star decontamination. Changes introduced were, for instance, a different binning method (often a rectangular grid using the Scott rule; Scott 1979), or skipping the Bayesian MP assignation and only performing a density based cell-by-cell field star removal. In this latter case, the DA works very similarly to the B07 algorithm.

An appropriate field star decontamination is of the utmost importance, since the cleaned cluster CMDs are used to estimate the cluster fundamental parameters.

\subsection{Synthetic CMD matching}

ASteCA derives the fundamental parameters of clusters by matching their observed CMDs with synthetic CMDs. In this work these are built using PARSEC v1.1 theoretical isochrones (Bressan et al. 2012, B12), and a lognormal initial mass function (IMF; Chabrier 2001). For a given age, metallicity, total mass, and binary fraction, a synthetic CMD is built from a stochastically sampled IMF down to the faintest portion of

12 http://WwW . astroml . org/examples/algorithms/plot_ bayesian_blocks.html 


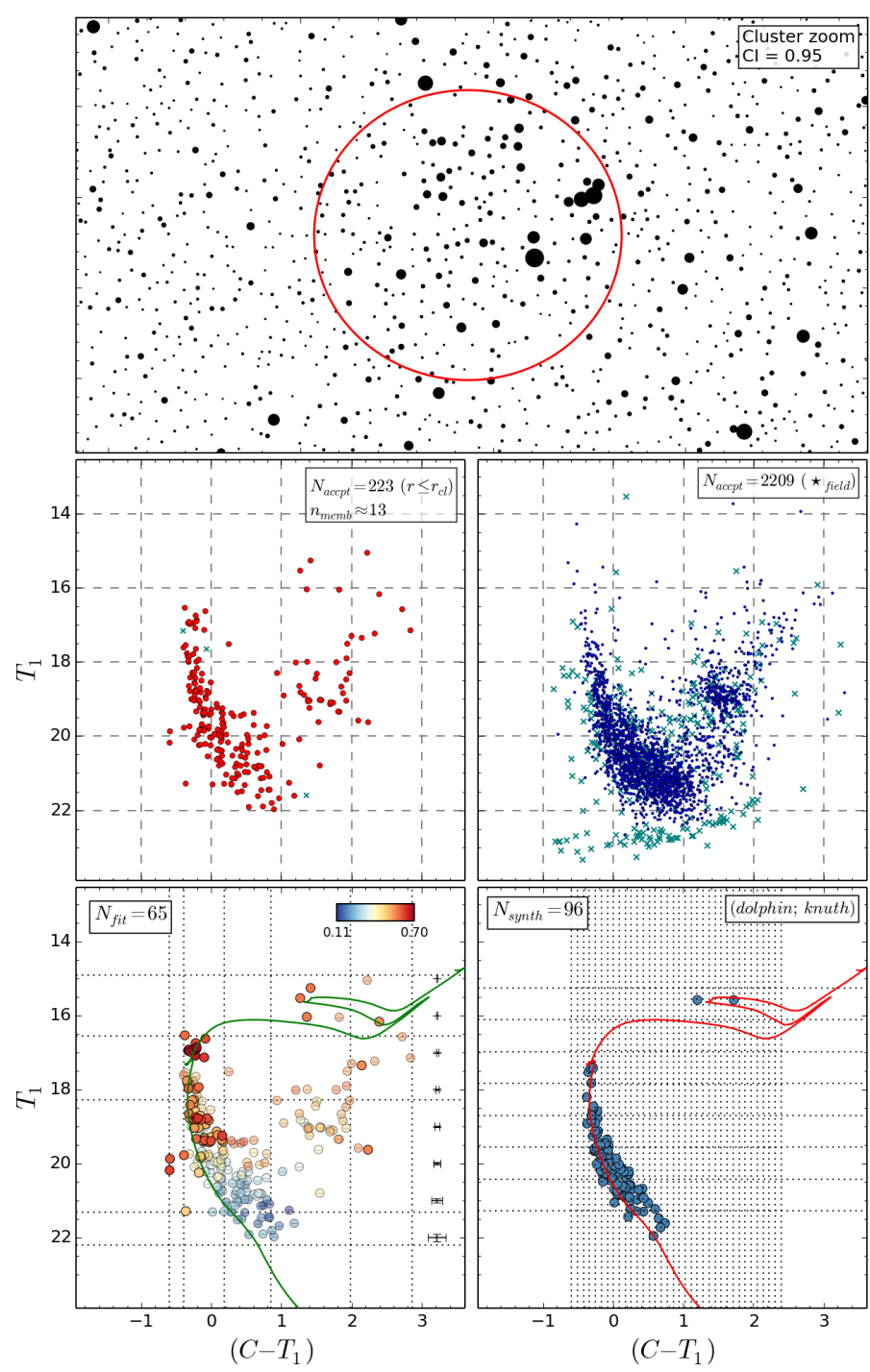

Fig. 2. Top: SMC-L62 cluster and surrounding field stars region. The adopted radius is indicated with a red circle. The CI value in the top right box is the contamination index; see Sect. 3.2. Middle: CMD of the cluster region for SMC-L62 (left), where $n_{\text {memb }}$ is the approximate number of cluster stars. The CMD of 10 combined surrounding field regions, each with an area equal to that of the cluster, is also shown (right). In both panels $N_{\text {accpt }}$ is the number of stars that were not rejected owing to their large photometric errors. Rejected stars with large errors are shown as pale green crosses. Bottom: cluster region after applying the DA (left); MPs vary according to the color bar at the top right. Dotted horizontal and vertical lines show the binning used to reject low MP stars cell by cell, as obtained via the Bayesian blocks method. Open circles (drawn semi-transparent) represent rejected stars. $N_{\text {fit }}$ is the number of stars kept unsubtracted by the cell-by-cell rejection. The best-fitted isochrone is overplotted with a green line. The respective generated synthetic CMD is shown in the right panel. $N_{\text {synth }}$ is the number of stars in the synthetic cluster CMD, and the dotted lines represent the binning obtained using Knuth's rule, applied in the synthetic cluster match process. The limiting magnitude of the synthetic CMD is taken from the limiting magnitude of the observed cluster (roughly $T_{1} \approx 22 \mathrm{mag}$, in this case).

the theoretical isochrone (defined by the metallicity and age values), shifted by a reddening and distance modulus. For a detailed description of the generation of synthetic CMDs from theoretical isochrones by ASteCA, see Paper I, Sect. 2.9.1. All the evolutionary tracks from the CMD service are currently supported along with three other IMFs.
The Poisson likelihood rate (PLR; Dolphin 2002) is employed to assess the match between the CMD of the cluster and a synthetic CMD, out of the $\sim 2 \times 10^{7}$ possible solutions (as shown in Table 3). The PLR statistic requires binning the CMD of the cluster and the synthetic CMDs generated according to the fundamental parameter ranges defined in Sect.3.1. We use Knuth's rule (Knuth 2006, also implemented via the astroML package) as the default binning method (see bottom right plot in Fig. 2). The inverted logarithmic form of the PLR can be written as

$L P L R=-2 \ln P L R=2 \sum_{i} m_{i}-n_{i}+n_{i} \ln \frac{n_{i}}{m_{i}}$,

where $m_{i}$ and $n_{i}$ are the number of stars in the $i$ th cell (twodimensional bin) of the synthetic and observed cluster's CMD, respectively. If for any given cell we have $n_{i} \neq 0$ and $m_{i}=0$, a very small number is used instead $\left(m_{i}=1 \times 10^{-10}\right)$ to avoid a mathematical inconsistency with the factor $\ln m_{i}$. Although ASteCA does not currently provide a goodness-of-fit estimator, uncertainties associated with the fitted fundamental parameters can be considered a coarse measure of the robustness of the fit. This parameter is to be added in upcoming versions of the code.

In Paper I the total mass parameter could not be estimated owing to the likelihood statistic used (Paper I, Eq. (11)). The LPR defined in Eq. (1) allows us to also consider the mass as a free parameter in the search of the best synthetic CMD. The total mass is estimated simultaneously along with the remaining fundamental parameters of a cluster, and no extra process is employed (for example, a mass-luminosity relation). Following the validation performed in Paper I for the metallicity, age, reddening, and distance, we present a similar study for the total mass in Appendix A. We demonstrate that the masses recovered by ASteCA for nearly 800 MASSCLEAN synthetic clusters - with masses ranging from $500 M_{\odot}$ to $2.5 \times 10^{5} M_{\odot}$ - are in excellent agreement with the masses used to generate them.

The determination of any fundamental parameter depends exclusively on the distribution and number of observed stars in the CMD of the cluster. Since we deal with five free fundamental parameters, a five-dimensional surface of solutions is built from all the possible synthetic CMDs. ASteCA applies a genetic algorithm (GA) on this surface to derive the fundamental parameters of the cluster. After the GA returns the optimal fundamental parameter values, uncertainties are estimated via a standard bootstrap technique. This process takes a significant amount of time to complete, since it involves running the GA several more times on a randomly generated cluster with replacement. Generating a new cluster with replacement means randomly picking stars one by one from the original cluster, where any star can be selected more than once. The process stops when the same number of stars as those present in the original cluster have been picked. We run the bootstrap 10 times per cluster, as it would be prohibitively costly timewise to run it - as would be ideal hundreds or even thousands of times. On average, the CMD of each cluster in our data set was compared to $\sim 1 \times 10^{6}$ synthetic CMDs, once the full process was completed.

\section{Errors in fitted parameters}

It is well known that a parameter given with no error estimation is meaningless from a physical standpoint (Dolphin 2002; Andrae 2010). Despite this, a detailed error treatment is often ignored in articles that deal with analysis of star clusters (Paunzen \& Netopil 2006).

As explained in Sect. 3.4, ASteCA employs a bootstrap method to assign standard deviations for each fitted parameter. 
The code simultaneously fits five free parameters within a wide range of allowed values, using only a two-dimensional space of observed data, i.e., the $T_{1}$ versus $\left(C-T_{1}\right) \mathrm{CMD}$, meaning uncertainties are somewhat large. We plan to upgrade the code to eventually allow more than just two observed magnitudes, extending the two-dimensional CMD analysis to an N-dimensional one. It is worth noting that, unlike manually set errors, these are statistically valid uncertainty estimates. This is an important point to make given that the usual by-eye isochrone fitting method not only disregards known correlations among all clusters parameters, it is also fundamentally incapable of producing a valid error analysis (Naylor \& Jeffries 2006). Any uncertainty estimate produced by eye serves only as a mere approximation, which is often biased toward smaller figures. The average logarithmic age error given in the literature, for example, is $0.16 \mathrm{dex}$, in contrast with the almost twice as large average value estimated by ASteCA (see below). In Fig. 3 we show the distribution of standard deviations of the five fundamental parameters fitted by the code for the entire sample.

The apparent dependence of the metallicity error with decreasing $[\mathrm{Fe} / \mathrm{H}]$ values arises from the fact that ASteCA uses $z$ values, where $[\mathrm{Fe} / \mathrm{H}]=\log \left(z / z_{\odot}\right)$ and $z_{\odot}=0.0152$. We use $z$ instead of $[\mathrm{Fe} / \mathrm{H}]$ because, first, this is the default form in which the evolutionary tracks are generated by the CMD service and, second, it is easier to work with the code when all parameters are strictly positive. The error in $z$ is $\sigma_{z} \approx 0.003$ for over $75 \%$ of the clusters analyzed. This means that when converting to $e_{[\mathrm{Fe} / \mathrm{H}]}$ using the relation

$\sigma_{[\mathrm{Fe} / \mathrm{H}]}=\sigma_{z} /[z \times \ln (10)]$,

the $z$ in the denominator makes $\sigma_{[\mathrm{Fe} / \mathrm{H}]}$ grow as it decreases, while $\sigma_{z}$ remains approximately constant. For very small $z$ values (e.g., $z=0.0001$ ), the logarithmic errors can surpass $\sigma_{[\mathrm{Fe} / \mathrm{H}]}=2$ dex. In those cases the error is trimmed to $2 \mathrm{dex}$, which is sufficient to cover the entire metallicity range.

There are no visible error trends for any of the fitted parameters, a desirable feature for any statistical method. If the uncertainty of a parameter varied (increase/decrease) with it, it would indicate ASteCA was introducing biases in the solutions.

Histograms to the right of Fig. 3 show the distribution of errors and their arithmetic means as a dashed red line. For $\sim 34 \%$ and $\sim 69 \%$ of the S/LMC clusters we have $\sigma_{[\mathrm{Fe} / \mathrm{H}]}<$ 0.2 dex. Approximately 53\% of the combined S/LMC sample show $\sigma_{\log (\text { age } / \mathrm{yr})}<0.1 \mathrm{dex}$. Error estimates for the remaining parameters are all within acceptable ranges. The uncertainties tend to increase for clusters of lower mass owing to the inherent stochasticity of the fitting process.

Errors could be lowered by applying different techniques: increase the number of models evaluated in the GA, reduce the value of the steps in the parameters' range, or increase the number of bootstrap runs. These methods, particularly the latter, extend the time needed to process each cluster. Limited computational time available requires a balance between the maximum processing power allocated to the calculations and the aimed precision. Error values presented here should then be considered a conservative upper limit.

\section{Comparision with published fundamental parameters}

We compare in Sect. 5.1 the parameter values obtained by ASteCA, with those taken from the papers that used the same $C T_{1}$ data sets (see Table 1) referred to as the literature. The parameters age, reddening, and mass are also analyzed in Sect 5.2
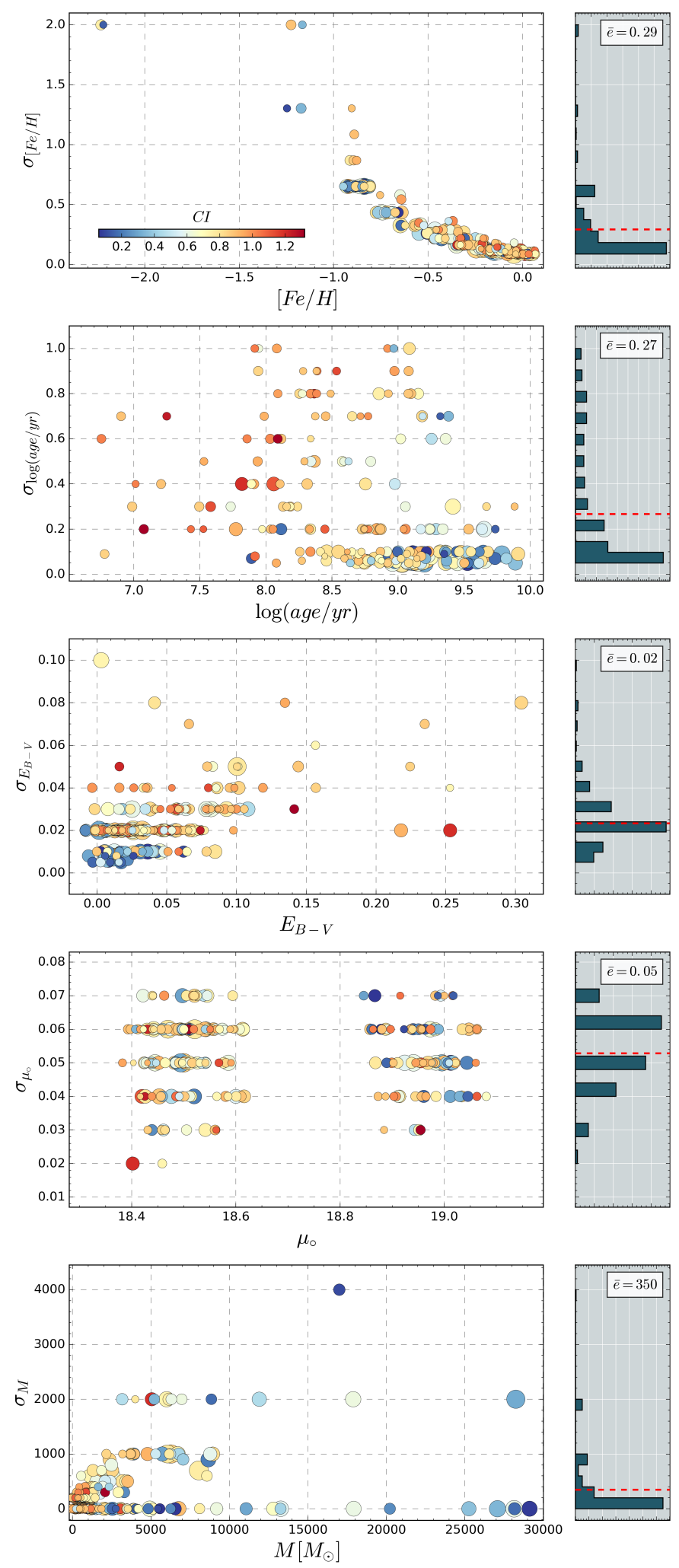

Fig. 3. Left: distribution of errors vs. the five parameters fitted by ASteCA. Colors are associated with the $C I$ (see bar in top plot); sizes are proportional to the actual cluster sizes. A small random scatter in the $x$-axis is added for clarity. Right: error histogram. The mean error value for each parameter is shown in the top right corner and drawn in the plot with a dashed red line.

for a subset of 142 star clusters that could be cross-matched with $U B V R I$ photometry results (see Table 2), referred to as the databases. 
G. I. Perren et al.: Astrophysical properties of star clusters in the Magellanic Clouds homogeneously estimated by ASteCA
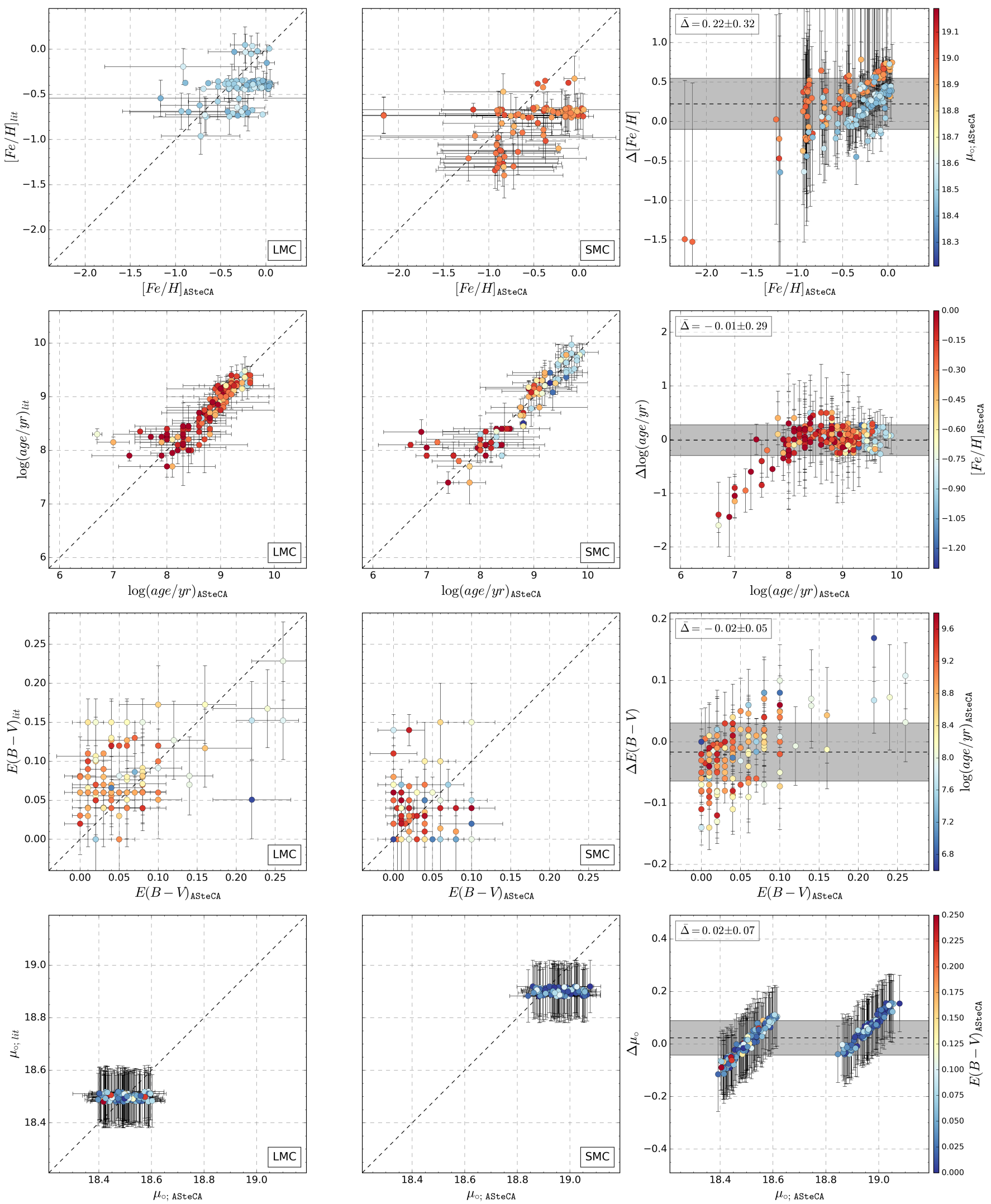

Fig. 4. Left column: parameters comparison for the LMC. Center column: idem for the SMC. Right column: BA plot with differences in the sense $\Delta=$ (ASteCA minus literature) for the combined S/LMC sample. A small random scatter is added to both axes for the metallicity and distance modulus plots. Mean and standard deviation of the differences, $\bar{\Delta}$, are shown as a dashed line and gray band, respectively; their values are indicated in the top left of the plot. Colors follow the bar at the right of the figure, for each row. Piatti (2011b) clusters with age information only are plotted with $E_{B-V}=0$. 


\subsection{Literature values}

We present ASteCA versus literature values for the metallicity, age, reddening, and distance modulus in Fig. 4. Left and central panels show the 1:1 identity line for both galaxies. Right panel diagram shows a Bland-Altman (BA) plot for our combined sample of clusters with the variation in the $x$-axis proposed by Krouwer (Bland \& Altman 1986; Krouwer 2008). The BA is also called a difference or Tukey Mean-Difference plot. In the original BA plot the default $x$-axis displays the mean values between the two methods being compared. The Krouwer variation changes the means for the values of one of those methods, called the reference. In our case, the reference is ASteCA so we use its reported values in the $x$-axis.

The ASteCA abundance estimates are slightly larger than those in the literature, shown as offsets in the upper panels of Fig. 4. On average, the offset is $\sim 0.27$ dex and $\sim 0.18$ dex for the S/LMC. This effect can be explained by two different processes in light of our knowledge that the best-fit CMD matching by ASteCA introduces no biases into the solutions. First, the ASteCA $z$ values are converted to the logarithmic form $[\mathrm{Fe} / \mathrm{H}]$ using a solar metallicity of $z_{\odot}=0.0152$ (Bressan et al. 2012). Literature values, on the other hand, are converted using a solar metal content of $z_{\odot}=0.019$ (Marigo et al. 2008) (usually rounded to 0.02 ). This means that ASteCA gives $[\mathrm{Fe} / \mathrm{H}]$ values larger by $\sim 0.1$ dex for any fitted isochrone of equivalent $z$ being compared, following

$$
\begin{aligned}
\Delta[\mathrm{Fe} / \mathrm{H}] & =[\mathrm{Fe} / \mathrm{H}]_{\mathrm{ASteCA}}-[\mathrm{Fe} / \mathrm{H}]_{\text {literature }} \\
& =\log (z / 0.0152)-\log (z / 0.019)=\log (0.019 / 0.0152) \\
& \approx 0.0969 \text { dex. }
\end{aligned}
$$

Second, the MC's star clusters are often studied using fixed metallicities. For example, $[\mathrm{Fe} / \mathrm{H}]$ values assumed in the 19 literature articles, are exactly -0.7 dex and -0.4 dex for $\sim 60 \%$ and $\sim 75 \%$ of S/LMC clusters. The by-eye fit is biased toward the assignment of these particular abundances because of the effect of confirmation bias. This effect present in the published literature has been studied recently by de Grijs et al. (2014) in relation to distance measurement reported for the LMC. Alternatively, the code is left free to fit metal contents in the entire range given. The $[\mathrm{Fe} / \mathrm{H}]$ values estimated by ASteCA are distributed mainly between -1 dex and 0 dex, but clustered closer to solar metal content than to lower metallicities. This fact, combined with the abovementioned effect of mostly fixed $[\mathrm{Fe} / \mathrm{H}]$ values used in the literature, makes the averaged difference in assigned metal content slightly positive. It also is responsible for the apparent linear growth of $\Delta[\mathrm{Fe} / \mathrm{H}]$ as $[\mathrm{Fe} / \mathrm{H}]_{\mathrm{ASteCA}}$ increases, as shown in Fig. 4. Researchers tend to fit isochrones by adjusting them to the lower envelope of the sequence of a cluster. This is carried out to avoid the influence of the binary sequence (located above and to the right of the single stars sequence in a CMD) in the fit, but it can also contribute to the selection of isochrones of smaller metallicity. The reason is that increasing the metal content of an isochrone moves it toward redder (greater color) values in a CMD; see, for example, Bressan et al. (2012), Fig. 15. These combined effects explain the offset found for the abundances. It is important to notice that neither effect is intrinsic to the best likelihood matching method used by the code.

The general dispersion between literature and ASteCA values can be quantized by the standard deviation of the $\Delta$ differences in the BA plot. This value is $\sim 0.32$ dex for the metallicity, in close agreement with the mean internal $[\mathrm{Fe} / \mathrm{H}]$ uncertainty found in Sect. 4. The ASteCA mean metallicities for the
$\mathrm{MCs}$ are $[\mathrm{Fe} / \mathrm{H}]_{\mathrm{SMC}} \simeq-0.52 \pm 0.44 \mathrm{dex}$ and $[\mathrm{Fe} / \mathrm{H}]_{\mathrm{LMC}} \simeq$ $-0.26 \pm 0.24$ dex. These averages are similar, within their uncertainties, to the following averages obtained using literature values: $[\mathrm{Fe} / \mathrm{H}]_{\mathrm{SMC}} \simeq-0.78 \pm 0.23 \mathrm{dex}$ and $[\mathrm{Fe} / \mathrm{H}]_{\mathrm{LMC}} \simeq-0.42 \pm$ 0.16 dex.

Ages show an overall good agreement with larger differences seen for a handful of young clusters. Ten clusters with either a shallow photometry, a low number of cluster stars, or heavily contaminated, present age differences larger than $\Delta \log ($ age $/ \mathrm{yr})>0.5 \mathrm{dex}$. These are referred to as outliers, and discussed in more depth in Appendix B. The mean $\Delta \log ($ age $/ y r)$ value for all S/LMC clusters, -0.01 dex as shown in the age BA plot of Fig. 4, points to an excellent agreement in $\log (\mathrm{age} / \mathrm{yr})$. Excluding outliers, this mean increases to $\sim 0.04$ dex. Similar to what was found for the metallicity, the $\sim 0.3$ dex dispersion is almost exactly the mean internal uncertainty found for errors assigned by the code.

The reddening distribution presents maximum $E_{B-V}$ values of $\sim 0.15 \mathrm{mag}$ and $\sim 0.3 \mathrm{mag}$ for the $\mathrm{S} / \mathrm{LMC}$, respectively. The $\Delta$ differences are well balanced with a standard deviation of 0.05 mag that is slightly larger than the 0.02 mag average uncertainty found for internal errors. We estimate average $E_{B-V}$ values for the $\mathrm{S} / \mathrm{LMC}$ of $0.03 \pm 0.03 \mathrm{mag}$ and $0.05 \pm 0.05 \mathrm{mag}$. These estimates are approximately a third of those used, for example, in the Hunter et al. (2003) study because our sample does not contain clusters in the regions of the MCs most affected by dust.

Distance moduli $\left(\mu_{\circ}\right)$ found by ASteCA show a clear displacement from literature values. This is expected, as the distance of the clusters from the literature is always assumed to be equivalent to the distance to the center of the corresponding galaxy. The distribution of $\mu_{\circ}$ values found by the code covers the entire range allowed in Sect. 3.1. This variation appears to have no substantial effect on any of the remaining parameters, something that could in principle be expected owing to the known correlations between them (see Paper I, Sect. 3.1.4). This reinforces the idea that using a fixed value for the distance modulus, as carried out in the literature, is a valid way of reducing the number of free variables at no apparent extra cost.

Masses are only assigned in Maia et al. (2013, M13) for its sample of 29 SMC clusters. The comparison with ASteCA is presented in Fig. 5, where the top panel shows a trend for ASteCA masses to be smaller than those from M13. This is particularly true for the clusters with the largest mass estimates - and the largest assigned errors - in M13: H86-97 (3300 $\left.\pm 1300 M_{\odot}\right)$ and H86-87 (3100 $\left.\pm 1700 M_{\odot}\right)$. The BA plot (bottom) shows that, on average, M13 masses are $\sim 500 \pm 700 M_{\odot}$ larger. To explain these differences, we need to compare the way total cluster masses are obtained by M13 and ASteCA.

The ASteCA package estimates masses by matching synthetic CMDs to that of the observed cluster region. The radius that delimits this region is formally equivalent to the tidal radius defined in (King 1962, see Sect. 3.2), but it is in practice smaller due to observational effects (i.e., Poisson errors in star counts, photometric incompleteness, and field stars contamination). A fraction of cluster members is thus left out of the analysis when this radius is used. We can prove via King's derivation of the number of members for a cluster (Eq. (18), King 1962), that this fraction is small except for highly underestimated radii $\left(r<0.5 r_{t}\right)$ and very low concentration clusters $\left(r_{t} / r_{c}<2\right)$. Massive clusters are largely unaffected by this process, while uncertainties for low-mass cluster masses are large enough that this effect can be safely ignored. To err on the side of caution, ASteCA masses should be considered lower estimates of the true present day cluster masses. 

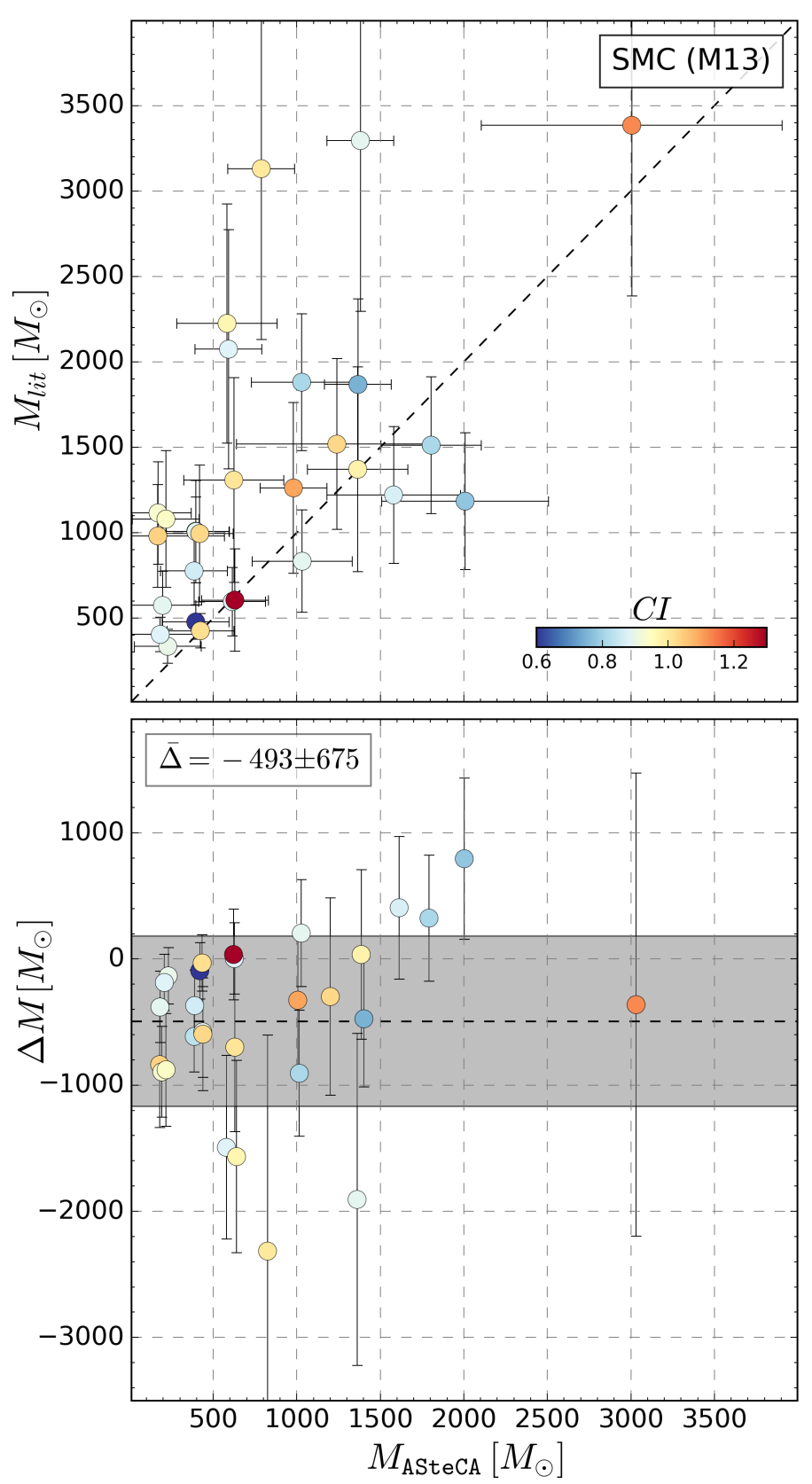

Fig. 5. Top: mass comparison for ASteCA vs. literature values. Bottom: BA plot with mean and standard deviation of the differences shown as a dashed horizontal line and a gray band, respectively. The mean and standard deviation values are shown in the top left corner.

In M13 cluster masses were determined using two methods, based on estimating a mass function via the $T_{1}$ luminosity function (LF). In both cases a field star cleaning process was applied. The first one employs a CMD decontamination procedure (Maia et al. 2010), and the second one cleans the LF of the cluster region by subtracting it a field star LF. Results obtained with these two methods are averaged to generate the final mass values. Although any reasonable field star cleaning algorithm should remove many or most of the foreground/background stars in a cluster region, some field stars are bound to remain. For heavily contaminated clusters this effect is determinant in shaping the clean CMD sequence, as cluster stars are very difficult to disentangle from contaminating field stars. Clusters in M13 are indeed heavily affected by field star contamination, as seen in Fig. 5, where colors follow their CIs. The minimum value is
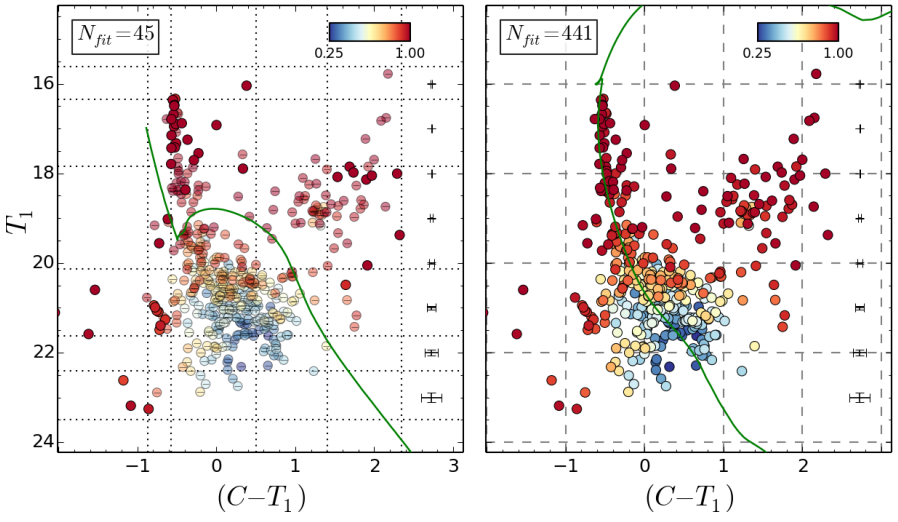

Fig. 6. Left: best-fit isochrone for B48 found by ASteCA when a cellby-cell removal is applied, following the Bayesian MP assignation (removed stars are drawn semi-transparent). Estimated age and total mass are $\log (\mathrm{age} / \mathrm{yr})=6.2 \pm 0.6$, and $M=400 \pm 200 M_{\odot}$. Right: best-fit isochrone found when no removal of stars is performed and the full cluster region is used in the search for the best synthetic cluster match. Estimated age and total mass are now $\log ($ age $/ \mathrm{yr})=7.5 \pm 0.3$, and $M=3000 \pm 900 M_{\odot}$.

$C I \simeq 0.55$, meaning all cluster regions are expected to contain, on average, more field stars than cluster stars. The presence of a large number of contaminating field stars not only makes the job much harder for the DA, it also implies that the LF is most likely overestimated. This leads, in the case of M13, to an overestimation of the total mass. In contrast, ASteCA assigns masses taking their values directly from the best match synthetic CMD. Field star contamination thus has a lower influence on the mass estimate of the code, which is only limited to how effective the DA is in cleaning the cluster region. We find in Appendix A that the code slightly underestimates masses by approximately $200 M_{\odot}$, for low-mass clusters. This is unrelated to the fraction of members lost from the employed radius (mentioned previously), since in this analysis we use the full tidal radius to delimit the MASSCLEAN synthetic clusters region. These combined effects explain the $\sim 500 M_{\odot}$ offset found for mass values, as shown in Fig. 5.

The case of B48 is worth mentioning, as it is the cluster with the largest total mass given in M13 (3400 $\left.\pm 1600 M_{\odot}\right)$. After removing possible field stars (see Sect. 3.3) low-mass stars disappear, and B48 is left only with its upper sequence $\left(T_{1}<18.4 \mathrm{mag}\right.$ ). This happens both in M13 (see Fig. C.8) and the ASteCA analysis (see left CMD in Fig. 6). The likelihood (Eq. (1)) sees then no statistical benefit in matching the CMD of the cluster with a synthetic CMD of similar age and mass, which contains a large number of low-mass stars. This leads the GA to select synthetic CMDs of considerably younger ages $(\log ($ age $/ y r)<7.0 \mathrm{dex})$ than that assigned in M13 $(\log (\operatorname{age} / \mathrm{yr})=7.9 \pm 0.05 \mathrm{dex})$, and with much lower mass estimates (see caption of Fig. 6). Age and mass values similar to those from M13 could only be found by the code if the DA was applied with no cell-by-cell removal of low MP stars, as shown in the right CMD of Fig. 6. This means that all field stars within the cluster region are used in the synthetic CMD matching process, which inevitably questions the reliability of the total mass estimate. Dealing with this statistical effect is not straightforward and will probably require an extra layer of modeling added to the synthetic CMD generation algorithm. As discussed in Appendix B, for a handful of outliers this effect also plays an important role in the significant age differences found between ASteCA and the literature. 


\subsection{Databases values}

We compare our results with those from seven articles - the databases or DBs - where a different photometric system was used; see Table 2. These DBs are separated into two groups: those in which the standard by-eye isochrone fitting method was applied, i.e., P99, P00, C06, and G10; and those in which integrated photometry was employed, i.e., H03, R05, and P12. A total of 142 clusters from our sample could be cross-matched. In those cases names were not available to perform the crossmatch, i.e., P00, P99, and R05, we employed a 20 arcsec finding radius, centered on the equatorial coordinates of the clusters.

Ages found by ASteCA versus the four isochrone-fit DBs ages, are shown in Fig. 7, left and center plot. In P99 and P00 SMC and LMC clusters, respectively, were analyzed using Bertelli et al. (1994) isochrones and fixed S/LMC metallicities $z=0.004,0.008$. While P99 derives individual reddening estimates based on red clump stars, P00 uses reddening values determined for 84 lines of sight in the Udalski et al. (1999) LMC Cepheids study. Both studies attempt to eliminate field star contamination following the statistical procedure presented in Mateo \& Hodge (1986). These DBs employed distance moduli of $18.65 \mathrm{mag}$ and $18.24 \mathrm{mag}$ for the S/LMC, which are approximately $\sim 0.25 \mathrm{mag}$ smaller than the canonical distances assumed for each Cloud. This has a direct impact on their obtained ages. In de Grijs \& Anders (2006) the authors estimate that had P00 used a value of $\mu_{0}=18.5$ mag instead, their ages would be $\sim 0.2-0.4$ dex younger; the same reasoning can be applied to the P99 age estimates. A similar conclusion is reached by Baumgardt et al. (2013). In the latter, the authors correct the age bias that arises in P00 owing to the small distance modulus used, by increasing P00 age estimates by 0.2 dex. This is incorrect because the ages should have been decreased by that amount. The P99 and P00 logarithmic ages are displaced on average from ASteCA values (in the sense ASteCA minus DB) by $-0.13 \pm 0.6$ dex and $0.37 \pm 0.5$ dex, respectively, as seen in the left plot of Fig. 7. In the case of P99, the distance modulus correction would bring the age values to an overall agreement with ASteCA, although with a large scatter around the identity line. The P00 age values, on the other hand, would end up $\sim 0.7$ dex below the code age estimates after such a correction. Such a large deviation is most likely due to the overestimated reddening values used by P00, as shown below.

In C06 311 SMC clusters were studied via isochrone fitting applying two methods: visual inspection and a Monte Carlo based $\chi^{2}$ minimization. The authors also employ a decontamination algorithm, making this the article that more closely resembles our work. Distance modulus is fixed to $\mu_{0}=18.9 \mathrm{mag}$, reddening and metallicity values of $E_{B-V}=0.08$ mag and $z=0.008$ are used, adjusted when necessary to improve the fit. The $[\mathrm{Fe} / \mathrm{H}]=-0.4$ dex abundance employed in $\mathrm{C} 06$ is closer to the $[\mathrm{Fe} / \mathrm{H}]=-0.52 \pm 0.44$ dex ASteCA average for the $\mathrm{SMC}$, than the canonical $[\mathrm{Fe} / \mathrm{H}]=-0.7$ dex value used in most works. Out of the seven DBs, C06 best matches the ASteCA $\log (\mathrm{age} / \mathrm{yr})$ values with a mean deviation from the identity line of $0.02 \pm 0.58$ dex.

The G10 authors analyzed over 1500 clusters with ages $<1 \mathrm{Gyr}$ in both Clouds via by-eye isochrone fitting. They assumed distance moduli of $(18.9,18.5) \mathrm{mag}$, and metallicities of $(0.004,0.008)$, for the S/LMC respectively. Reddening was adjusted also by eye on a case-by-case basis. This DB presents a systematic bias, where smaller logarithmic ages are assigned compared to our values, with an approximate deviation of $\Delta \log (\mathrm{age} / \mathrm{yr}) \simeq 0.23 \pm 0.46$ (ASteCA minus G10). This is consistent with the results found in Choudhury et al. (2015) (see Fig. 5), and later confirmed in Piatti et al. (2015b,a). The G10 authors do not apply any decontamination method, instead they plot a sample of surrounding field stars over the cluster region. The lack of a proper statistical removal of contaminating foreground/background stars can skew the isochrone fit.

As shown in Fig. 7 (center plot), these four DBs present a clear age-extinction bias compared to ASteCA values with the maximum density located around $\Delta E_{B-V} \simeq-0.07 \mathrm{mag}$ and $\Delta \log ($ age $/ \mathrm{yr}) \simeq 0.3 \mathrm{dex}$. This degeneracy was found in $\mathrm{Pa}-$ per I (Table 3 ) to have the largest correlation value, meaning it is the process most likely to affect isochrone fit studies. The trend is most obvious for $\mathrm{P} 00$, where a large average reddening of $E_{B-V} \simeq 0.14 \mathrm{mag}$ (de Grijs \& Anders 2006) was employed, compared to the mean value found by ASteCA. In Fig. 7 on the right, we see the same plot but generated by subtracting literature from ASteCA age estimations. The aforementioned bias is basically nonexistent here, pointing to a consistent assignation of reddening and ages by the code.

In Appendix $C$ we show CMDs for the 153 cross-matched clusters across these four DBs. Clusters that present the largest age discrepancies between ASteCA and the DBs are those in which the same effect mentioned in Appendix B takes place. A good example of this is SMC-L39, as shown in Figs. C.9 and C.18 for C06 and G10, respectively.

Our age and mass estimates are also compared with three DBs, i.e., H03, R05, and P12, which used integrated photometry (see Table 2). Only H03 and P12 obtained total mass values, and these are analyzed in Sect. 5.2.1.

The H03 authors studied approximately 1000 clusters: 196 and 748 in the S/LMC via $U B V R$ integrated photometry. Ages were assigned based on the Starburst99 model (Leitherer et al. 1999), assuming metallicities, distance moduli, and average $E_{B-V}$ values of $(0.004,0.008),(18.94,18.48) \mathrm{mag}$, and $(0.09$, $0.13) \mathrm{mag}$, for the S/LMC, respectively. Masses were derived from $M_{V}$ absolute magnitude and a mass-luminosity relation. This article represents, as far as we are aware, the largest published database of MCs cluster masses to date.

In R05 the models GALEV (Anders \& Fritze-v. Alvensleben 2003) and Starburst99 were used, combined with three metallicities, i.e., $(0.004,0.008)$ and $(0.001,0.004,0.008)$ in each model respectively, to obtain five age estimates for 195 SMC clusters. Reddening values were assigned using fixed age ranges, following Harris \& Zaritsky (2004). We averaged all reddeningcorrected ages for each matched cluster and assigned an error equal to the midpoint between the lowest and highest error.

The P12 authors used the same data set from $\mathrm{H} 03$ to analyze 920 LMC clusters through their MASSCLEAN colors $_{\text {. }}$

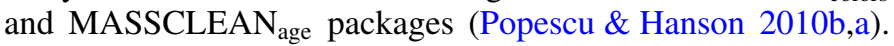
Metallicities were fixed to $z=0.008$, while reddening was taken from G10 when available or fixed to $E_{B-V}=0.13 \mathrm{mag}$, as in H03. Ages and masses from duplicated entries in the P12 sample are averaged in our analysis.

As seen in Fig. 8, H03 underestimates ages for younger clusters. This effect was registered in de Grijs \& Anders (2006, see Fig. 1), which the authors assigned to the photometry conversion carried out in H03. Average log(age/yr) dispersion between $\mathrm{H} 03$ and ASteCA is $0.44 \pm 0.56$ dex. The same happens for P12 ages, albeit with a smaller $\log ($ age/yr) dispersion of $\sim 0.35 \pm 0.44$ dex. The P12 authors compared their own age estimates with $\mathrm{H} 03$ (see P12, Fig. 8). They find a systematic difference with $\mathrm{H} 03$, where MASSCLEAN ages are larger for clusters with $\log ($ age $/ y r)<8$ dex. In our case, most of the clusters cross-matched with P12 are older than 8 dex, with P12 

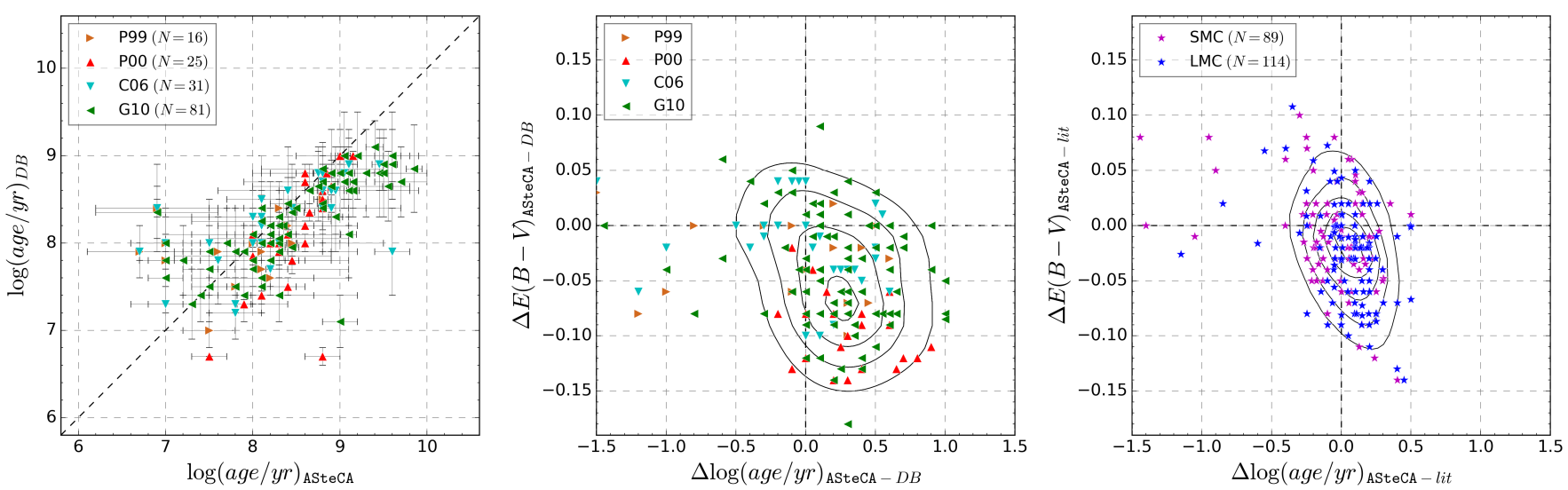

Fig. 7. Left: age comparison for DBs that used the isochrone fit method vs. ASteCA, where $N$ is the number of clusters cross-matched in each DB. Center: Delta plot (ASteCA minus DB) showing differences between reddening and age. Curves represent regions of iso-densities after fitting a two-dimensional Gaussian kernel. Right: same as previous plot, for ASteCA minus literature values for both Clouds.

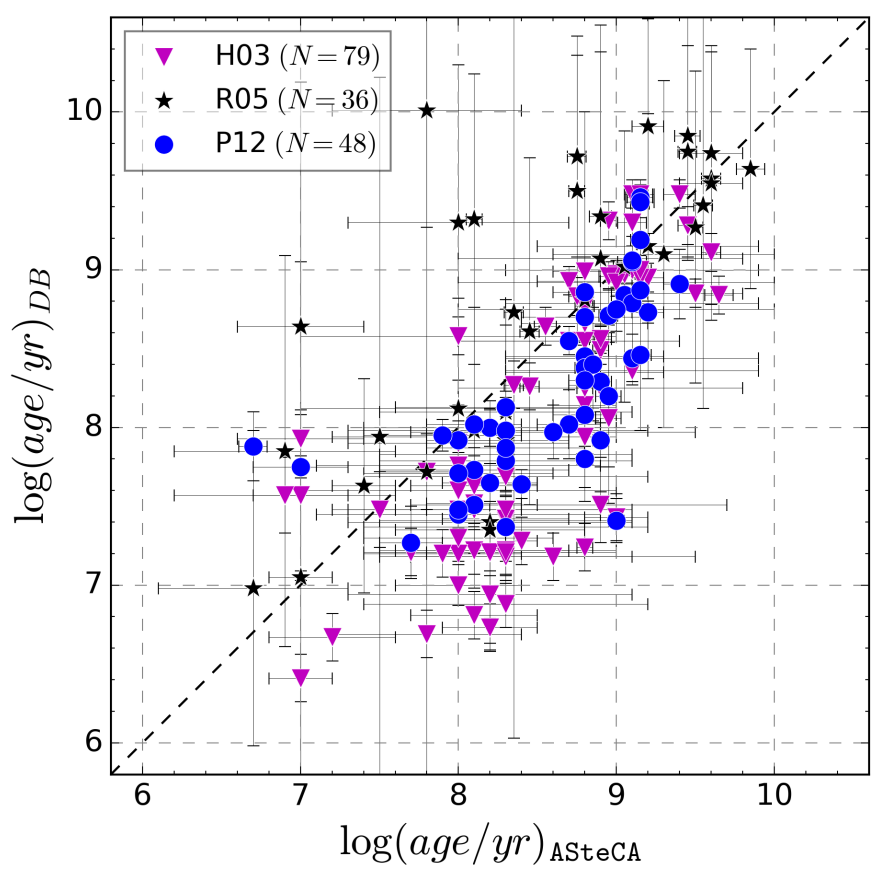

Fig. 8. Age comparison for integrated photometry DBs vs. ASteCA. $N$ is the number of clusters cross-matched in each DB.

ages located mostly below the identity line. This bias toward smaller age estimates by P12 is consistent with what was found in Choudhury et al. (2015). Contrary to the results found for H03 and P12, the R05 study slightly underestimates ages compared to ASteCA with a mean $\log ($ age/yr) dispersion of $-0.25 \pm 0.63$ dex. The standard deviation is the largest for the three integrated photometry DBs. In R05 the authors mention the lack of precision in their age measurements from the use of integrated colors and the lack of constraints for the metallicity.

Expectedly, the four isochrone fit studies analyzed previously show a more balanced distribution of ages around the 1:1 relation, in contrast with the integrated photometry DBs. Ages taken from integrated photometry studies are known to be less accurate and should be taken as a rather coarse approximation to the true values. As shown in P12, integrated colors present large scatters for all age values, leading inevitably to degeneracies in the final solutions. The added noise by contaminating field stars is also a key issue, as this noise is very difficult to remove properly from integrated photometry data. A single overly bright field star can substantially modify the observed luminosity of the cluster, leading to incorrect estimates of its parameters (Baumgardt et al. 2013; Piatti 2014). A detailed analysis of some of the issues encountered by integrated photometry studies and of the accuracy of their results is presented in Anders et al. (2013).

\subsubsection{Integrated photometry masses}

Cross-matching the $\mathrm{H} 03$ and P12 DBs with a maximum search radius of 20 arcsec results in 670 unique LMC clusters. In the top row of Fig. 9 we show the differences in mass and age estimates for both DBs. The leftmost diagram contains all the crossmatched clusters, while the other diagrams are sectioned by mass ranges. The three mass regions show how the age-mass correlation - an older large cluster is incorrectly identified as a much younger and less massive one or vice versa - strongly affects estimates between these DBs. The mean value $\overline{\Delta M_{\text {log }}}$ (logarithmic mass difference) decreases from $0.8 \pm 0.5$, to $0.3 \pm 0.5$, to $-0.4 \pm 1$; in the low, medium, and high-mass regions defined in Fig. 9, respectively. Masses go from being overestimated a factor of $\sim 6$ by P12 in the low-mass region, to being underestimated by a factor of $\sim 2.5$ in the high-mass region. Where P12 estimates higher masses, it also assigns larger ages by more than 1.5 dex. Conversely, in the high average mass region, $\mathrm{P} 12$ ages can reach values up to 3 dex lower than H03. There are five clusters with H03 masses higher than $2.5 \times 10^{5} M_{\odot}$ in this cross-matched sample, all incorrectly assigned a low age and mass by P12. These five LMC globular clusters are NGC 1916, NGC 1835, NGC 1786, NGC 1754, and NGC 1898. The P12 paper assigns masses below $2000 M_{\odot}$ in all cases.

Cross-matching the H03 and P12 DBs with our sample, results in a set of 127 clusters. The age-mass positive correlation that was obvious for $\mathrm{H} 03$ and $\mathrm{P} 12$, is not present when we compare DBs masses and ages estimates with those from ASteCA, as seen in the bottom row of Fig. 9. In Fig. 10 we show masses for the 127 cross-matched clusters in these DBs versus their logarithmic mass differences. The mean standard mass difference $\Delta M\left(=M_{\mathrm{ASteCA}}-M_{\mathrm{DBs}}\right)$ is $\sim 40 \pm 1700 M_{\odot}$ for $M_{\mathrm{DBs}}<5 \times 10^{3} M_{\odot}$, and $\sim-400 \pm 2500 M_{\odot}$ for $M_{\mathrm{DBs}}<10^{4} M_{\odot}$, which points to a very reasonable scatter around the identity 


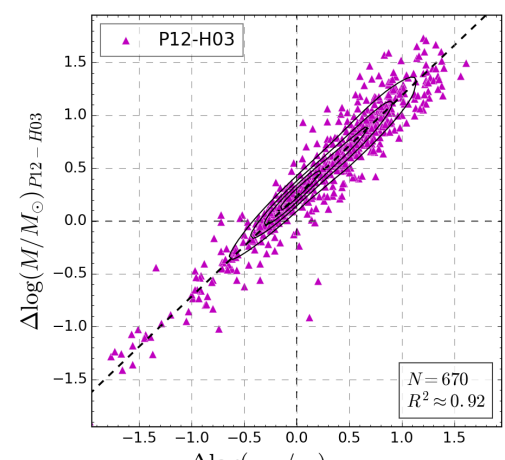

$\Delta \log (\text { age } / y r)_{P 12-H 03}$

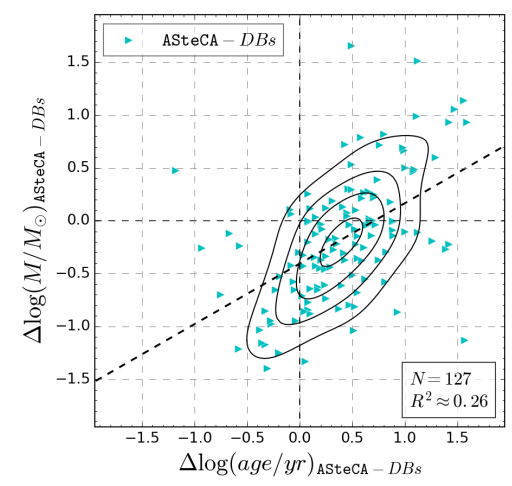

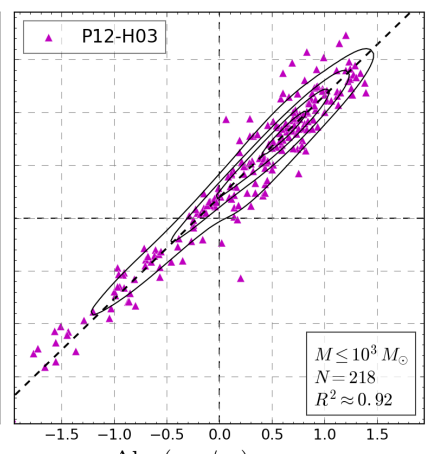

$\Delta \log (\text { age } / y r)_{P 12-H 03}$

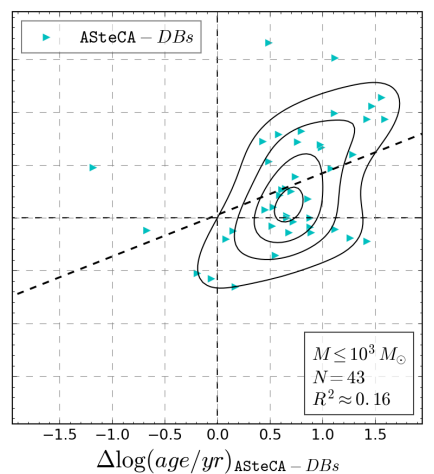

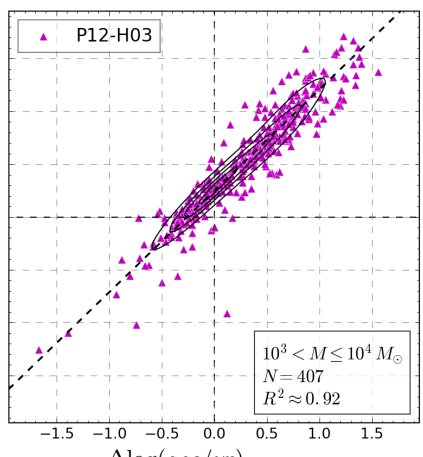

$\Delta \log (\text { age } / y r)_{P 12-H 03}$

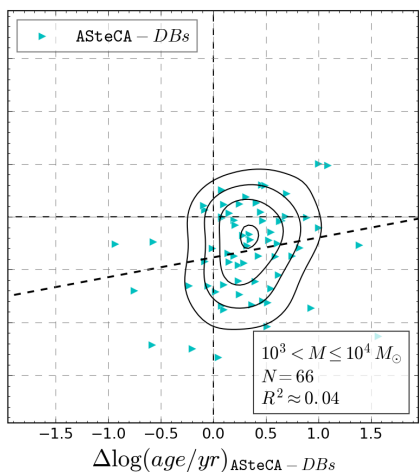

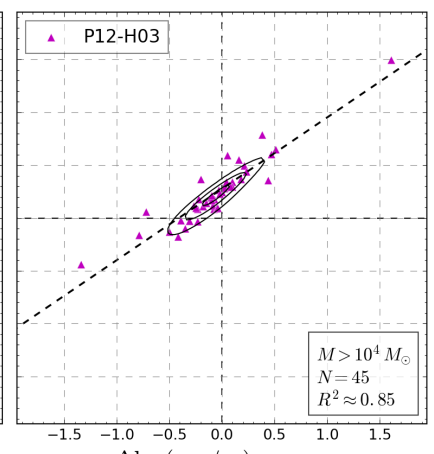

$\Delta \log (\text { age } / y r)_{P 12}-H 03$

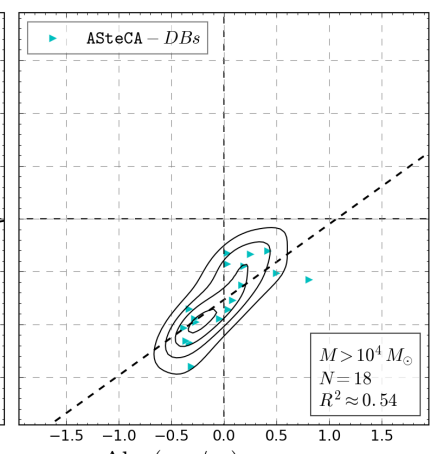

$\Delta \log (\text { age } / y r)_{\mathrm{ASteCA}}-D B s$

Fig. 9. Top row: differences plot $\log (\mathrm{age} / \mathrm{yr})$ vs. $\log \left(M / M_{\odot}\right)$, for $670 \mathrm{P} 12$ and $\mathrm{H} 03$ cross-matched clusters, in the sense P12 minus H03. A twodimensional Gaussian kernel density estimate is shown as iso-density black curves. The dashed line is the result of the best-fit linear regression; the $R^{2}$ coefficient is shown in the bottom left box. The leftmost diagram shows all 670 clusters processed together; the remaining diagrams are divided by mass ranges. Bottom: idem, for ASteCA vs. DBs (where DBs represent the combined H03 and P12 sample) for the 127 cross-matched clusters.

line. Although the standard deviation values are somewhat large, this is expected for a set of clusters in this mass range. As stated in Baumgardt et al. (2013) and P12, clusters with $M \lesssim$ $5 \times 10^{3}-10^{4} M_{\odot}$ tend to have their estimated integrated photometry masses largely dominated by stochastic processes.

We see in Fig. 10 that the larger the DB mass, the larger the logarithmic difference with ASteCA. Values for $\overline{\Delta M_{\log }}$ in the medium and high-mass regions of Fig. 10 mean that DB masses are between two and six times larger than ASteCA masses. The most discrepant case is that of SMC-NGC 419 - left out of the right plot for clarity, which shows a mass of $3.9 \times 10^{5} M_{\odot}$ by $\mathrm{H} 03$, and $2.8 \times 10^{4} M_{\odot}$ by the code.

After exploring several possible processes that could induce this systematic mass difference between our estimates and those from integrated photometry studies, we concluded that the effect of stellar crowding in our Washington photometry is responsible. The likelihood defined in Eq. (1) is a binned statistic. This means that stars not accounted for in the CMD bias the mass estimation, as it depends on the number of observed stars. The larger the number of stars that is lost because of this process, the larger the underestimation of mass for a given observed cluster. To test the above hypothesis, we requested the HST data used in Goudfrooij et al. (2014) to analyze NGC 419. The ACS/WFC instrument - from where this data comes from has a much higher resolution compared to that from our Washington photometry ( $0.05 \mathrm{arcsex} /$ pixel versus $0.274 \mathrm{arcsec} / \mathrm{pixel})$, so we can expect a substantially lower percentage of stars lost. The authors estimate a mass of $\sim 2.4 \times 10^{5} M_{\odot}$ using a Salpeter (1955) IMF, which could be as low as $\sim 1.5 \times 10^{5} M_{\odot}$ if a more recent IMF was used. We analyzed this data set with ASteCA fixing all parameters to the values given in Goudfrooij et al. with the exception of the mass that was left to vary up to $3 \times 10^{5} M_{\odot}$.
A cut on $F 555 W=23$ mag was imposed to minimize the impact of faint stars lost due to crowding. The mass found this way for NGC 419 is $2.5 \times 10^{5} M_{\odot}$, a very similar value to the average of $\mathrm{H} 03$ and Goudfrooij et al. $\left(2.6 \times 10^{5} M_{\odot}\right)$. It is thus clear that stellar crowding in our low resolution photometry is responsible for the systematic difference in estimated masses.

Even though ASteCA corrects synthetic clusters using a completeness function, which is approximated from the LF of the observed cluster, this only affects the faintest synthetic stars. For heavily crowded clusters (especially if they are observed with low resolution) this underestimates the loss of brighter stars, which intensifies as one moves closer to the center of the cluster (Mateo 1988), hence underestimating its mass. Future releases of the code will allow the user to input a manual completeness function, ideally obtained through proper artificial star tests (see, e.g., Aparicio \& Gallart 1995). Mass values obtained in the present study for massive clusters should therefore be considered lower estimates of their true value.

\section{Fundamental parameters in the analyzed database}

We present a summary of the distribution of the five fundamental parameters obtained with ASteCA, for the 239 MCs clusters in our catalog. A method is devised in Sect. 6.1 to allow an unbiased analysis of the estimated values and the results are presented in Sect. 6.2. Reddenings, distances, and total masses do not span sufficiently large ranges to account for the values found in the MCs. Their distribution thus only characterize the state of those clusters in this catalog. On the other hand, metallicities and ages obtained cover a wide range in both galaxies. Their distribution can be regarded as a representative randomized sample 
G. I. Perren et al.: Astrophysical properties of star clusters in the Magellanic Clouds homogeneously estimated by ASteCA
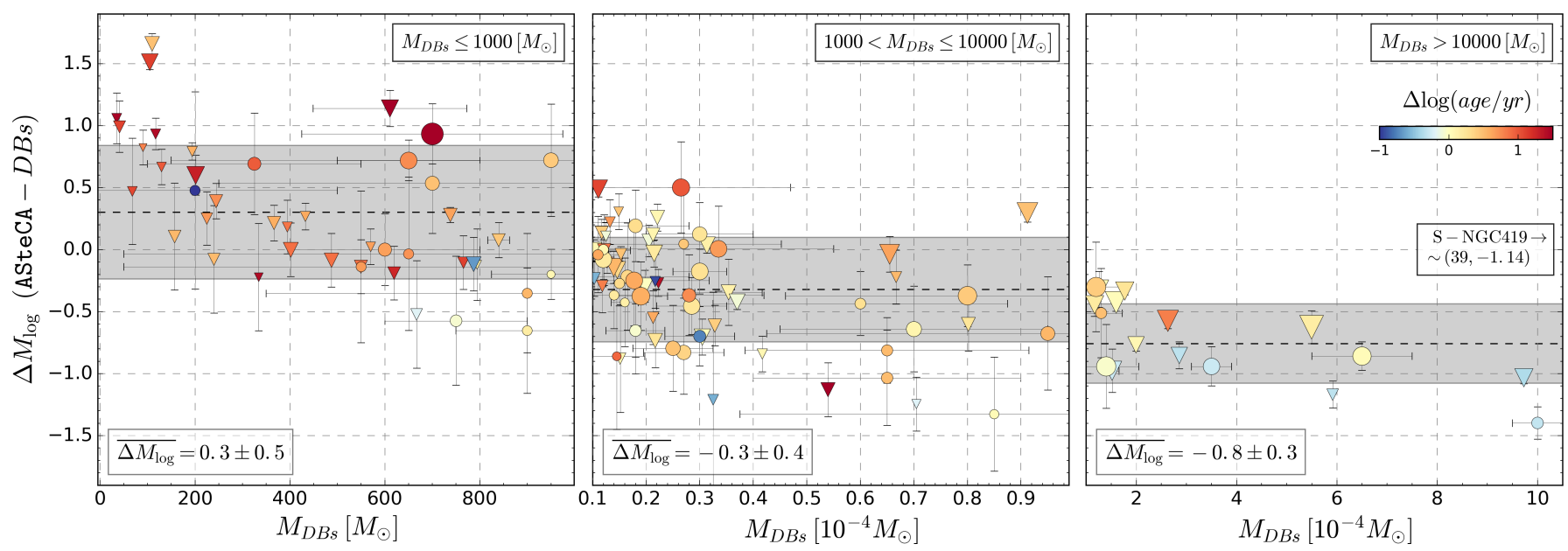

Fig. 10. Left: BA mass plot, showing the differences between estimated masses in the H03 and P12 DBs and the code, in the sense ASteCA minus DB; symbols as in Fig. 8. Only DB masses $\leq 10^{3} M_{\odot}$ are shown here. Colors are assigned according to the difference in log(age/yr) estimation of each cluster (ASteCA minus DB, color bar is shown in the right plot), while sizes are proportional to the actual sizes in parsecs. The horizontal dashed line $\left(\overline{\Delta M_{\log }}\right)$ is the mean of the combined logarithmic differences. The gray band is the $\pm 1 \sigma$ region for this mean. Center: same as previous plot, now showing DB mass values in the range $10^{3}-10^{4} M_{\odot}$. Right: same as previous plot, for DB mass values $>10^{4} M_{\odot}$.

of the cluster system in the MCs. Their relationship is studied in Sect. 6.3.

\subsection{Method}

Histograms are used to derive a large number of properties in astrophysical analysis, for example, the star formation history (SFH) of a galaxy. Their widespread use notwithstanding, the generation of a histogram is affected by well-known issues (see Silverman 1986; Simonoff \& Udina 1997). Different bin widths and anchor positions can make histograms built from the same data look utterly dissimilar. In the worst cases, completely spurious substructures may appear, leading the analysis toward erroneous conclusions. We bypass these issues by constructing an adaptive (variable) Gaussian kernel density estimate (KDE) in one and two dimensions, using the standard deviations of the parameters as bandwidth estimates. The formulas for both KDEs are

$$
\begin{aligned}
& K D E_{1 \mathrm{D}}(x)=\frac{1}{N \sqrt{2 \pi}} \sum_{i=1}^{N} \frac{1}{\sigma_{i}} \mathrm{e}^{-\frac{\left(x-x_{i}\right)^{2}}{2 \sigma_{i}^{2}}}, \\
& K D E_{2 \mathrm{D}}(x, y)=\frac{1}{2 \pi N} \sum_{i=1}^{N} \frac{1}{\sigma_{x i} \sigma_{y i}} \mathrm{e}^{-\frac{1}{2}\left(\frac{\left(x-x_{i}\right)^{2}}{\sigma_{x i}}+\frac{\left(y-y_{i}\right)^{2}}{\sigma_{y i}^{2}}\right)},
\end{aligned}
$$

where $N$ is the number of observed values, $x_{i}$ is the $i$ th observed value of parameter $x$, and $\sigma_{x i}$ its assigned standard deviation (same for $y_{i}$ and $\sigma_{y i}$ ). The 1D version of these KDEs is similar to the "smoothed distribution" used in the Rafelski \& Zaritsky (2005) study of SMC clusters. Using standard deviations as bandwidth estimates means that the contribution to the density map of parameters with large errors is smoothed (spread out) over a large portion of the domain. Precise parameter values, on the other hand, contribute to a much more narrow region.

Replacing one- and two-dimensional histogram analyses with these KDEs has two immediate benefits: first, it frees us from having to select an arbitrary bandwidth value, which is the most important component of a $\mathrm{KDE}$ ); second, it naturally incorporates errors obtained for each parameter into its probability density function.

\subsection{Distribution of parameters within the observed ranges}

Figures 11 and 12 show 1D and 2D density maps constructed via Eqs. (4) and (5). A distinct period of cluster formation is visible in the LMC around $\sim 5 \mathrm{Gyr}$, which culminated $\sim 1.3 \mathrm{Gyr}$ ago. A similar but less pronounced peak is seen for the SMC with a drop in cluster formation around $\sim 2$ Gyr. Height differences between the SMC and LMC KDEs is related to the relative decline in cluster formation. While the LMC sharply drops to almost zero from $\sim 1$ Gyr to present times, the SMC shows a softer descent with smaller peaks around $\sim 250 \mathrm{Myr}$ and 130 Myr. The well-known age gap in the LMC between 3-10 Gyr (Balbinot et al. 2010) is present and is visible as a marked drop in the $K D E_{\log (\text { age } / y r)}$ curve at $\sim 9.5$ dex.

The 2D KDE age-metallicity map shows how spread out these values are for clusters in the SMC, compared to those in the LMC. Although the SMC abundance reaches substantially lower values, the right $1 \mathrm{D} \mathrm{KDE}$ reveals $[\mathrm{Fe} / \mathrm{H}]$ peaks between 0 dex and -0.2 dex for clusters in both Clouds.

The age-mass 2D map shows a clustering around younger ages and smaller masses for the LMC. The SMC cluster seen in the bottom right corner is $\operatorname{HW} 42\left(\alpha=1^{\mathrm{h}} 01^{\mathrm{m}} 08^{\mathrm{s}}, \delta=\right.$ $-74^{\circ} 04^{\prime} 25^{\prime \prime}$ [J2000.0]), a small cluster (radius $<20 \mathrm{pc}$ ) located close the SMC center. Though its position in the map is somewhat anomalous, the $1 \sigma$ error in its age and mass estimates could move it to $\log ($ age $/ \mathrm{yr}) \simeq 9.4$ and $\log \left(M / M_{\odot}\right) \simeq 2.6$. This cluster is classified as a possible emissionless association by Bica \& Schmitt (1995). There is a tendency in both Clouds for the mass and size of the clusters to grow with estimated age, as expected (due to the mass-to-light ratio increase with age; see Popescu et al. 2012, Sect. 4). As seen in Fig. 12 (top), the 1D KDEs of the distance moduli are well behaved and normal in their distribution. A Gaussian fit to these curves results in bestfit values of $18.96 \pm 0.08 \mathrm{mag}$ and $18.49 \pm 0.08 \mathrm{mag}$ for the S/LMC. Literature mean distances are thus properly recovered. Reddening values are much more concentrated in the sample of SMC clusters around $E_{B-V} \approx 0.015$ mag. The LMC reddenings are dispersed below $E_{B-V} \approx 0.1 \mathrm{mag}$ with a shallower peak located at $\sim 0.03$ mag. 

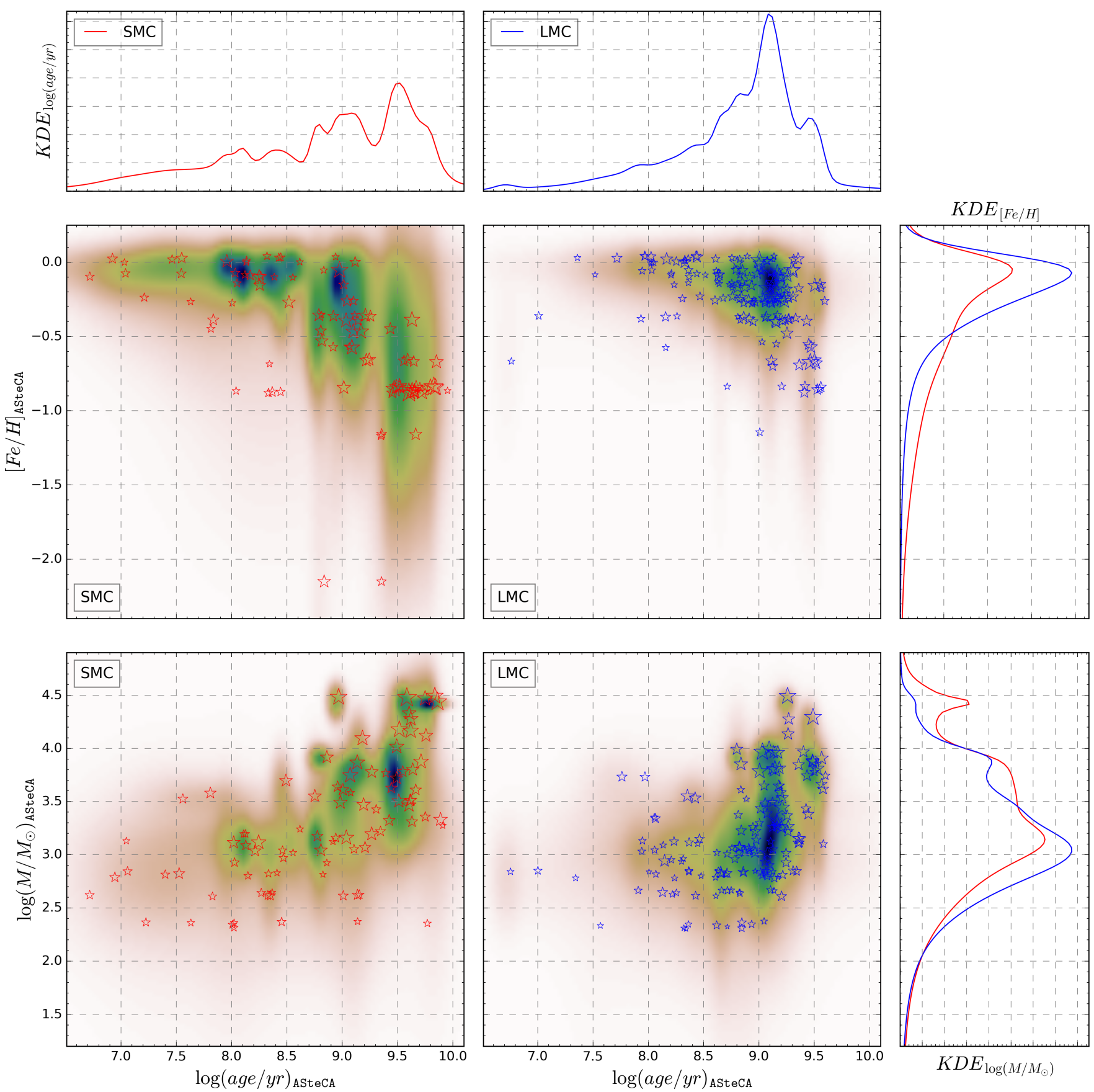

Fig. 11. Adaptive Gaussian KDEs for age, metallicity, and mass. The top and right plots are 1D KDEs; center plots are 2D KDEs. Observed clusters are plotted as red and blue stars for the S/LMC, respectively. Sizes are scaled according to the radius of each cluster. A small scatter is introduced for clarity.

\subsection{Age-metallicity relation}

The age-metallicity relation (AMR) of a stellar system is an essential tool to learn about its chemical enrichment evolution. In Piatti (2010) an AMR method was devised using age bins of different sizes to take age errors into account. It was applied to derive AMRs in Piatti \& Geisler (2013) and adapted to obtain star cluster frequency distributions in Piatti (2013). We propose a new method based on the KDE technique described in Sect. 6 with a number of advantages over previous methods. Mathematical details are given in Appendix D, where the method is applied to generate AMRs using literature age and metallicity values.
The ASteCA AMRs for the S/LMC can be seen in Fig. 13 as red and blue continuous lines, respectively. Stars show the position of all clusters in our sample with sizes scaled according to their radii. Shaded regions represent the $1 \sigma$ standard deviations of the AMRs, spanning a $[\mathrm{Fe} / \mathrm{H}]$ width of $\sim 0.2$ dex for the entire age range, for both Clouds. The blue (top) and red (bottom) vertical segments in the top plot are the bin edges determined for each age interval by Knuth's algorithm. The final AMR functions are mostly unaffected by the chosen binning method. Using Knuth's algorithm results in $\sim 11$ age intervals of widths between 0.35 and $1 \mathrm{Gyr}$, as shown in Fig. 13. If instead we use 100 intervals of 

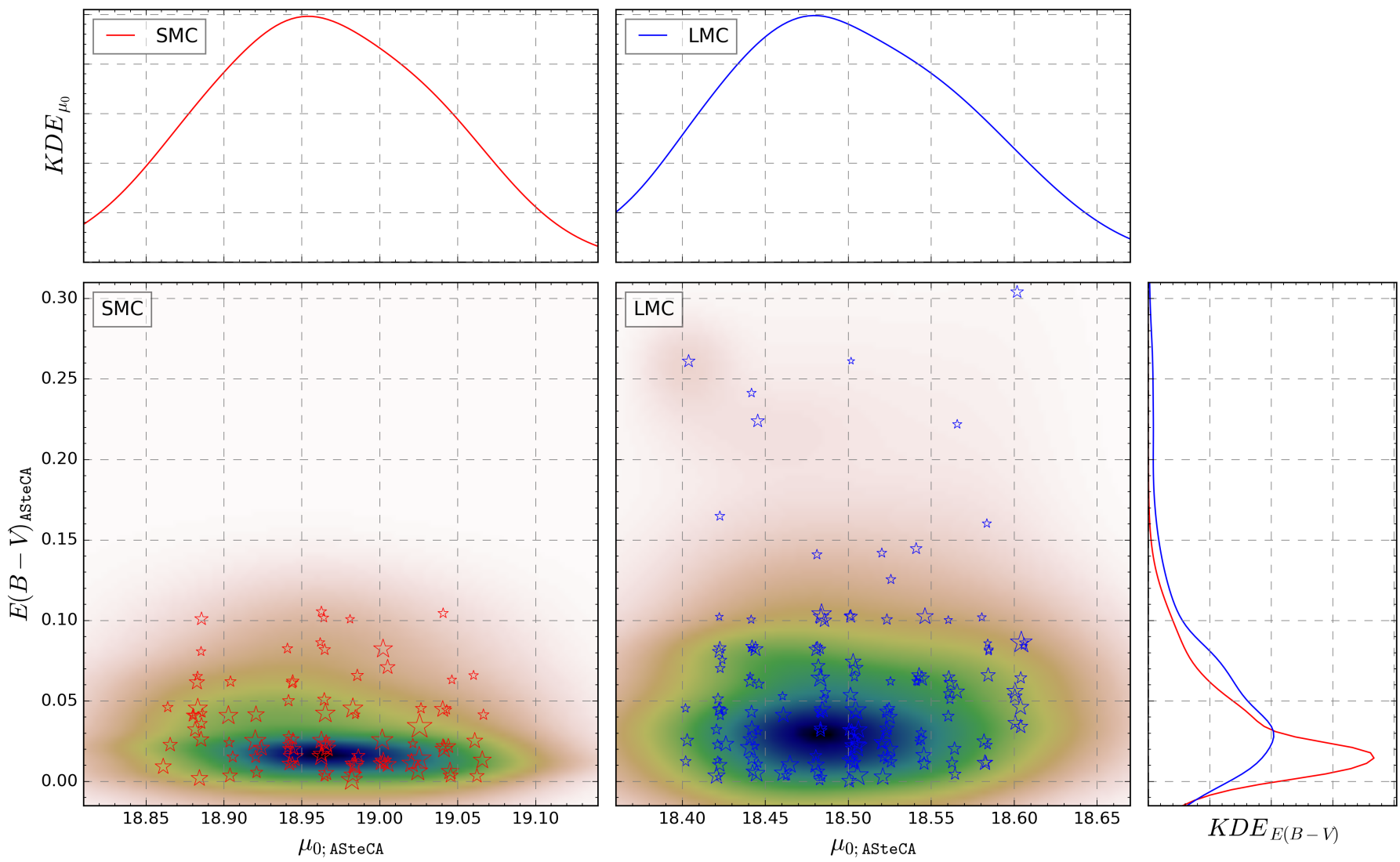

Fig. 12. Same as Fig. 11 for $E_{(B-V)}$ and distance modulus.

$\sim 0.1$ Gyr width, only the SMC curve is perturbed in the region Age $<800 \mathrm{Myr}$ where $[\mathrm{Fe} / \mathrm{H}]$ values are raised by $\sim 0.1 \mathrm{dex}$. If the two SMC clusters with very low metal abundances $([\mathrm{Fe} / \mathrm{H}]<$ -2 dex) are excluded from our data, the AMR moves upward in the $[\mathrm{Fe} / \mathrm{H}]$ axis by less than 0.05 dex, for ages below $500 \mathrm{Myr}$.

Several chemical evolution models and empirical AMRs are found in published articles. We show AMR functions presented in 12 other works in the center and bottom plots of Fig. 13. These external studies constitute a representative sample of the following different methods and data used over the past 20 yr: Pagel \& Tautvaisiene (1998, PT98; bursting models), Geha et al. (1998, G98; closed-box model with Holtzman SFH), Harris \& Zaritsky (2004, HZ04), Carrera et al. (2008b, C08a; average of four disk frames), Carrera et al. (2008a, C08b; average of 13 frames), Harris \& Zaritsky (2009, HZ09), Noël et al. (2009, N09; 5th degree polynomial fit to the AMRs of their three observed regions), Tsujimoto \& Bekki (2009, TB09; 1: no merger model, 2: equal mass merger, 3: one to four merger), Rubele et al. (2012, R12; four tiles average), Cignoni et al. (2013, C13; B: Bologna, C: Cole), Piatti \& Geisler (2013, PG13), and Meschin et al. (2014, M14; 0: field LMC0, 1: field LMC1, 2: field LMC2). Details of how these AMRs were constructed can be consulted in each reference. All of the abovementioned articles used field stars for the obtention of their AMRs. This is, as far as we are aware, the first work in which the AMR function for both galaxies is derived entirely from observed star clusters.

The AMRs' trend coincides with what has already been found, namely that the metallicity increases for younger ages (particularly below 3 Gyr). On average, our AMR estimates are displaced slightly toward more metal rich values. Most $[\mathrm{Fe} / \mathrm{H}]$ values in the external studies are obtained using a solar metallicity of $z_{\odot}=0.019$, while we used the more recent value $z_{\odot}=0.0152$. As was shown in Sect. 5.1, this difference means $[\mathrm{Fe} / \mathrm{H}]$ estimates will be $\sim 0.1$ dex smaller for external studies.

For the LMC galaxy, Fig. 13 center plot, we see a marked drop in metallicity from $\sim-0.45$ dex beginning around $3.8 \mathrm{Gyr}$ and ending 3 Gyr ago at -0.6 dex. The M14-0 curve seems to reproduce this behavior, but shifted $\sim 0.8 \mathrm{Gyr}$ toward younger ages. This high metallicity value of the AMR of the LMC at its old end, is caused by the three clusters with $[\mathrm{Fe} / \mathrm{H}] \approx-0.2$ dex located beyond $\sim 3.5 \mathrm{Gyr}$; the oldest ages estimated by ASteCA. Without any older clusters available in the LMC, it is hard to assess whether this is a statistically significant feature of the AMR. After the drop, there is a steep climb from 3 Gyr to 2 Gyr reaching almost $[\mathrm{Fe} / \mathrm{H}] \sim-0.3 \mathrm{dex}$, and then a sustained shallower increase up to the estimated present day metal content of $\sim-0.15$ dex. Our average metallicity value for present day clusters coincides reasonably well with the PT98 bursting model, which shows nonetheless a very different rate of increase from 2 Gyr to present times. The C08a AMR, while lacking finer details, provides a better match for this age range. The HZ09 and G98 AMRs differ the most not only from the ASteCA AMR, but from the rest of the group.

The SMC ASteCA AMR is shown along with 10 published AMRs in Fig. 13, bottom. The peak around $~ 7.5$ Gyr predicted by TB09 in its two merger models (1:1, and 1:4 merger) is not visible in our AMR. The ASteCA AMR remains largely stable around a value of $[\mathrm{Fe} / \mathrm{H}] \simeq-0.9$ dex until approximately $3 \mathrm{Gyr}$ ago, where the rate of growth increases considerably. From that point up to the present day, the average metallicity for clusters in the SMC grows by $\sim 0.4$ dex. The abundance increase for ages $<3$ Gyr is only reproduced by the PT98 model and the HZ04 function. The PT98 model starts diverging from our AMR 

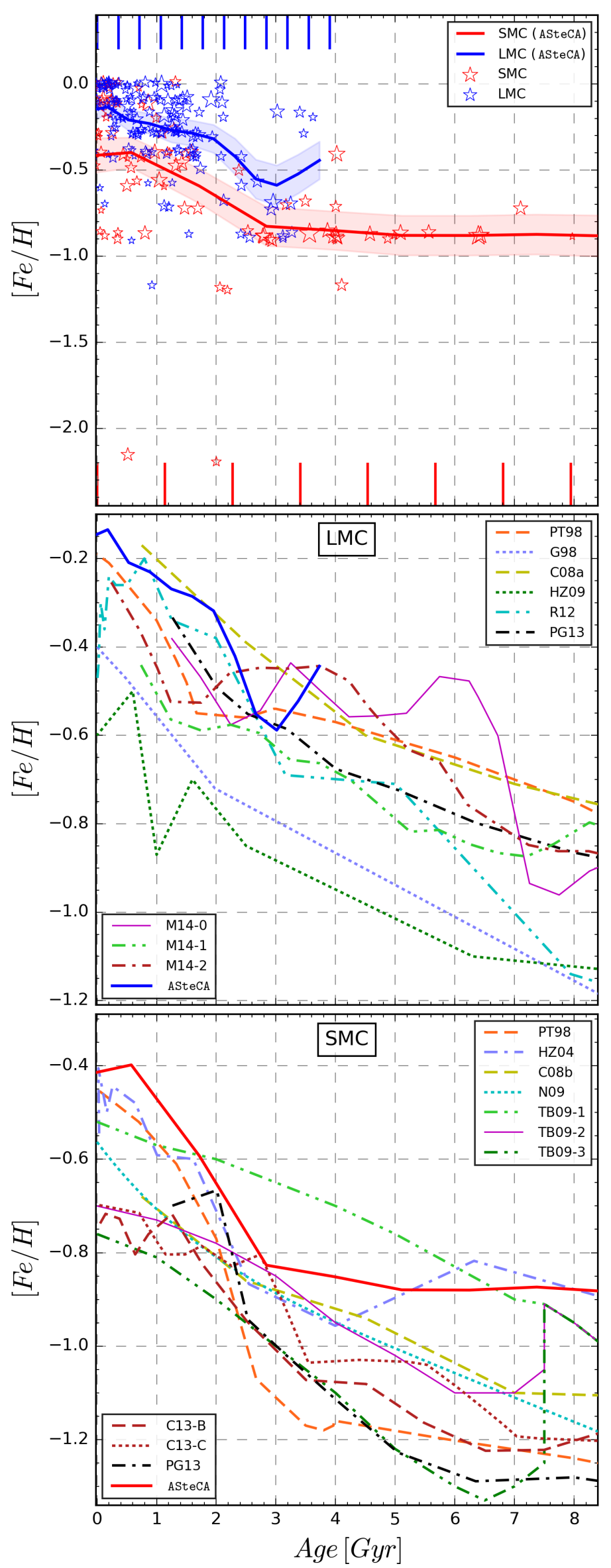

Fig. 13. ASteCA age-metallicity relation for the $\mathrm{S} / \mathrm{LMC}$ (red/blue solid lines). See text in Sect. 6.3 for more details. at $\sim 2$ Gyr until a gap of $\sim 0.4 \mathrm{dex}$ in $[\mathrm{Fe} / \mathrm{H}]$ is generated. In contrast, the HZ04 curve remains much closer to our own throughout the entire age range. These two AMRs estimate a present day metallicity that is very close to the $[\mathrm{Fe} / \mathrm{H}] \approx-0.4$ dex value estimated by ASteCA.

Overall, our AMRs cannot be explained by any single model or empirical AMR function and are best reproduced by a combination of several. A similar result was found in Piatti \& Geisler (2013), although their field stars AMRs are significantly different from ours, mainly for the SMC. It is important to remember that the ASteCA AMRs are averaged over the structure of both Magellanic Clouds. In Fig. 1 we showed that our set of clusters covers a large portion of the surface of these galaxies. If more clusters were available so that the AMRs could be estimated by $\mathrm{S} / \mathrm{LMC}$ sectors, it is possible that different results would arise.

\section{Summary and conclusions}

We presented a homogeneous catalog of 239 star clusters in the Large and Small Magellanic Clouds, observed with the Washington photometric system. The clusters span a wide range in metallicity and age and are spatially distributed throughout both galaxies. The fundamental parameters metallicity, age, reddening, distance modulus, and total mass were determined using the ASteCA package. This tool allows the automated processing of the positional and photometric data of a cluster, resulting in estimates of both its structural and intrinsic/extrinsic properties. As shown in Paper I, the advantages of using ASteCA include reproducible and objective results, along with a proper handling of the uncertainties involved in the synthetic cluster matching process. This permits the generation of a truly homogeneous catalog of observed clusters with their parameters fully recovered. Our resulting catalog is complete for all the analyzed parameters, including metallicity and mass, which are two properties often assumed or not obtained at all.

Internal errors show no biases present in our determination of fundamental parameters, as shown in Sect. 4. The analysis of our results in Sect. 5.1 demonstrate that the assigned values for the clusters are in good agreement with published literature, which used the same Washington photometry. Metallicity was the most discrepant parameter with the ASteCA $[\mathrm{Fe} / \mathrm{H}]$ values on average $\sim 0.22$ dex larger than those present in the literature. Half of this difference is due to the solar abundance used in this work. The remaining $\sim 0.1$ dex is explained by the confirmation bias effect in most cluster studies, which assume canonical $[\mathrm{Fe} / \mathrm{H}]$ values rather than derive them through statistically valid means. We also compared our results with articles that used different photometric systems in Sect. 5.2. While the age differences in this case are somewhat larger, they can be mostly explained by effects outside the code.

We performed, in Sect. 5.2.1, a detailed comparative study of masses obtained through integrated photometry studies with our own estimates from CMD analysis. Although the estimation is reasonable for smaller clusters, mass values are systematically underestimated for larger clusters because of the effect of stellar crowding in our own photometry.

A method for deriving the distribution of any fundamental parameter - or a combination of two of them - is presented in Sect. 6.1. This method takes into account the information contained by the uncertainties, which is often excluded from the analysis. By relying on Gaussian kernels, the method is robust and independent of ad hoc binning choices. An agemetallicity relation is derived for cluster systems in both galaxies 
in Sect. 6.3. The AMRs generated cannot be fully matched by any model or empirical determination found in recent literature.

We demonstrated that the ASteCA package is able to produce proper estimations for the fundamental parameters of observed star clusters within the limitations imposed by the photometric data. A necessary statistically valid error analysis can be performed, thanks to its built-in bootstrap error assignment method. The tool is proven capable of operating almost entirely unassisted on large databases of clusters. This is an increasingly essential feature of any astrophysical analysis tool, given the growing importance of big data and the necessity to conduct research on large astronomical data sets.

Acknowledgements. The authors are very much indebted with the anonymous referee for the helpful comments and suggestions that contributed to greatly improving the manuscript. G.I.P. would like to thank the help and assistance provided throughout the redaction of several portions of this work by D. Hunter, A. E. Dolphin, M. Rafeslski, D. Zaritsky, T. Palma, F. F. S. Maia, B. Popescu, H. Baumgardt, J. C. Forte, and P. Goudfrooij. This research has made use of the VizieR catalogue access tool, operated at CDS, Strasbourg, France (Ochsenbein et al. 2000). This research has made use of "Aladin sky atlas" developed at CDS, Strasbourg Observatory, France (Bonnarel et al. 2000; Boch \& Fernique 2014). This research has made use of NASA's Astrophysics Data System. This research made use of the Python language v2.7 (van Rossum 1995) and the following packages: NumPy ${ }^{13}$ (Van Der Walt et al. 2011); SciPy $^{14}$ (Jones et al 2001); Astropy ${ }^{15}$, a community-developed core Python package for Astronomy (Astropy Collaboration et al. 2013); scikit-learn ${ }^{16}$ (Pedregosa et al. 2011); matplotlib $^{17}$ (Hunter 2007). This research made use of the Tool for OPerations on Catalogues And Tables (TOPCAT, Taylor 2005) ${ }^{18}$.

\section{References}

Anders, P., \& Fritze-v. Alvensleben, U. 2003, A\&A, 401, 1063

Anders, P., Kotulla, R., De Grijs, R., \& Wicker, J. 2013, ApJ, 778, 138

Andrae, R. 2010, ArXiv e-prints [arXiv: 1009.2755]

Aparicio, A., \& Gallart, C. 1995, AJ, 110, 2105

Astropy Collaboration, Robitaille, T. P., Tollerud, E. J., et al. 2013, A\&A, 558, A33

Balbinot, E., Santiago, B. X., Kerber, L. O., Barbuy, B., \& Dias, B. M. S. 2010, MNRAS, 404, 1625

Baumgardt, H., Parmentier, G., Anders, P., \& Grebel, E. K. 2013, MNRAS, 430, 676

Bertelli, G., Bressan, A., Chiosi, C., Fagotto, F., \& Nasi, E. 1994, A\&AS, 106, 275

Bevington, P. R., \& Robinson, D. K. 2003, in Data reduction and error analysis for the physical sciences, 3rd edn. (Boston, MA: McGraw-Hill)

Bica, E. L. D., \& Schmitt, H. R. 1995, ApJS, 101, 41

Bica, E., Bonatto, C., Dutra, C. M., \& Santos, J. F. C. 2008, MNRAS, 389, 678

Bland, J. M., \& Altman, D. G. 1986, Lancet, 1, 307

Boch, T., \& Fernique, P. 2014, in Astronomical Data Analysis Software and Systems XXIII, eds. N. Manset, \& P. Forshay, ASP Conf. Ser., 485, 277

Bonatto, C., \& Bica, E. 2007, MNRAS, 377, 1301

Bonnarel, F., Fernique, P., Bienaymé, O., et al. 2000, A\&AS, 143, 33

Bressan, A., Marigo, P., Girardi, L., et al. 2012, MNRAS, 427, 127

Burstein, D., \& Heiles, C. 1982, AJ, 87, 1165

Canterna, R. 1976, AJ, 81, 228

Carrera, R., Gallart, C., Aparicio, A., et al. 2008a, AJ, 136, 1039

Carrera, R., Gallart, C., Hardy, E., Aparicio, A., \& Zinn, R. 2008b, AJ, 135, 836

Chabrier, G. 2001, ApJ, 554, 1274

Chiosi, E., Vallenari, A., Held, E. V., Rizzi, L., \& Moretti, A. 2006, A\&A, 452, 179

Choudhury, S., Subramaniam, A., \& Piatti, A. E. 2015, AJ, 149, 52

Cignoni, M., Cole, A. A., Tosi, M., et al. 2013, ApJ, 775, 83

de Grijs, R., \& Anders, P. 2006, MNRAS, 366, 295

\footnotetext{
13 http: //WwW . numpy.org/

14 http://www. scipy.org/

15 http://www . astropy.org/

16 http://scikit-learn.org/

17 http://matplotlib.org/

18 http://wwW.starlink.ac.uk/topcat/
}

de Grijs, R., \& Bono, G. 2015, AJ, 149, 179

de Grijs, R., Wicker, J. E., \& Bono, G. 2014, AJ, 147, 122

Dias, W. S., Alessi, B. S., Moitinho, A., \& Lépine, J. R. D. 2002, A\&A, 389, 871 Dolphin, A. E. 2002, MNRAS, 332, 91

Elson, R. A. W., Sigurdsson, S., Davies, M., Hurley, J., \& Gilmore, G. 1998, MNRAS, 300, 857

Geha, M. C., Holtzman, J. A., Mould, J. R., et al. 1998, AJ, 115, 1045

Geisler, D. 1996, AJ, 111, 480

Geisler, D., \& Sarajedini, A. 1999, AJ, 117, 308

Geisler, D., Bica, E., Dottori, H., et al. 1997, AJ, 114, 1920

Geisler, D., Piatti, A. E., Bica, E., \& Claria, J. J. 2003, MNRAS, 341, 771

Glatt, K., Grebel, E. K., \& Koch, A. 2010, A\&A, 517, A50

Goudfrooij, P., Girardi, L., Kozhurina-Platais, V., et al. 2014, ApJ, 797, 35

Grocholski, A. J., \& Sarajedini, A. 2003, MNRAS, 345, 1015

Harris, J., \& Zaritsky, D. 2004, AJ, 127, 1531

Harris, J., \& Zaritsky, D. 2009, AJ, 138, 1243

Haschke, R., Grebel, E. K., \& Duffau, S. 2011, AJ, 141, 158

Hills, S., von Hippel, T., Courteau, S., \& Geller, A. M. 2015, AJ, 149, 94

Hunter, D. A., Elmegreen, B. G., Dupuy, T. J., \& Mortonson, M. 2003, AJ, 126, 1836

Hunter, J. D. 2007, Comp. Sci. Eng., 9, 90

Jones, E., Oliphant, T., Peterson, P., et al. 2001, SciPy: Open source scientific tools for Python [Online; accessed 2016-06-21]

Kharchenko, N. V., Piskunov, A. E., Röser, S., Schilbach, E., \& Scholz, R.-D. 2005, A\&A, 440, 403

King, I. 1962, AJ, 67, 471

Knuth, K. H. 2006, ArXiv Physics e-prints [arXiv:physics/0605197]

Krouwer, J. S. 2008, Stat. Med., 27, 778

Leitherer, C., Schaerer, D., Goldader, J. D., et al. 1999, ApJS, 123, 3

Maia, F. F. S., Corradi, W. J. B., \& Santos, Jr., J. F. C. 2010, MNRAS, 407, 1875

Maia, F. F. S., Piatti, A. E., \& Santos, J. F. C. 2013, MNRAS, 437, 2005

Marigo, P., Girardi, L., Bressan, A., et al. 2008, A\&A, 482, 883

Mateo, M. 1988, ApJ, 331, 261

Mateo, M., \& Hodge, P. 1986, ApJS, 60, 893

Meschin, I., Gallart, C., Aparicio, A., et al. 2014, MNRAS, 438, 1067

Naylor, T., \& Jeffries, R. D. 2006, MNRAS, 373, 1251

Netopil, M., Paunzen, E., \& Carraro, G. 2015, A\&A, 582, A19

Nidever, D. L., Monachesi, A., Bell, E. F., et al. 2013, ApJ, 779, 145

Noël, N. E. D., Aparicio, A., Gallart, C., et al. 2009, ApJ, 705, 1260

Ochsenbein, F., Bauer, P., \& Marcout, J. 2000, A\&AS, 143, 23

Pagel, B. E. J., \& Tautvaisiene, G. 1998, MNRAS, 299, 535

Palma, T., Clariá, J. J., Geisler, D., Piatti, A. E., \& Ahumada, A. V. 2013, A\&A, 555, A131

Paunzen, E., \& Netopil, M. 2006, MNRAS, 371, 1641

Pedregosa, F., Varoquaux, G., Gramfort, A., et al. 2011, J. Machine Learning Res., 12, 2825

Perren, G. I., Vázquez, R. A., \& Piatti, A. E. 2015, A\&A, 576, A6

Phelps, R. L., Janes, K. A., \& Montgomery, K. A. 1994, AJ, 107, 1079

Piatti, A. E. 2010, A\&A, 513, L13

Piatti, A. E. 2011a, MNRAS, 416, L89

Piatti, A. E. 2011b, MNRAS, 418, L40

Piatti, A. E. 2011c, MNRAS, 418, L69

Piatti, A. E. 2012, A\&A, 540, A58

Piatti, A. E. 2013, MNRAS, 437, 1646

Piatti, A. E. 2014, MNRAS, 445, 2302

Piatti, A. E., \& Bica, E. 2012, MNRAS, 425, 3085

Piatti, A. E., \& Geisler, D. 2013, AJ, 145, 17

Piatti, A. E., Bica, E., Geisler, D., \& Claria, J. J. 2003a, MNRAS, 344, 965

Piatti, A. E., Geisler, D., Bica, E., \& Claria, J. J. 2003b, MNRAS, 343, 851

Piatti, A. E., Sarajedini, A., Geisler, D., Seguel, J., \& Clark, D. 2005, MNRAS, 358, 1215

Piatti, A. E., Sarajedini, A., Geisler, D., Clark, D., \& Seguel, J. 2007a, MNRAS, 377,300

Piatti, A. E., Sarajedini, A., Geisler, D., Gallart, C., \& Wischnjewsky, M. 2007b, MNRAS, 382, 1203

Piatti, A. E., Sarajedini, A., Geisler, D., Gallart, C., \& Wischnjewsky, M. 2007c, MNRAS, 381, L84

Piatti, A. E., Geisler, D., Sarajedini, A., Gallart, C., \& Wischnjewsky, M. 2008, MNRAS, 389, 429

Piatti, A. E., Geisler, D., Sarajedini, A., \& Gallart, C. 2009, A\&A, 501, 585

Piatti, A. E., Clariá, J. J., Bica, E., et al. 2011a, MNRAS, 417, 1559

Piatti, A. E., Clariá, J. J., Parisi, M. C., \& Ahumada, A. V. 2011b, Publ. Astron. Soc. Pac., 123, 519

Piatti, A. E., de Grijs, R., Ripepi, V., et al. 2015a, MNRAS, 454, 839

Piatti, A. E., de Grijs, R., Rubele, S., et al. 2015b, MNRAS, 450, 552

Pietrzynski, G., \& Udalski, A. 1999, Acta Astron., 49, 157

Pietrzynski, G., \& Udalski, A. 2000, Acta Astron., 50, 337

Popescu, B., \& Hanson, M. M. 2009, AJ, 138, 1724 
A\&A 602, A89 (2017)

Popescu, B., \& Hanson, M. M. 2010a, ApJ, 724, 296

Popescu, B., \& Hanson, M. M. 2010b, ApJ, 713, L2

Popescu, B., Hanson, M. M., \& Elmegreen, B. G. 2012, ApJ, 751, 122

Rafelski, M., \& Zaritsky, D. 2005, AJ, 129, 2701

Rubele, S., Girardi, L., Kozhurina-Platais, V., Goudfrooij, P., \& Kerber, L. 2011, MNRAS, 414, 2204

Rubele, S., Kerber, L., Girardi, L., et al. 2012, A\&A, 537, A106

Salpeter, E. E. 1955, ApJ, 121, 161

Scargle, J. D., Norris, J. P., Jackson, B., \& Chiang, J. 2013, ApJ, 764, 167

Schlafly, E. F., \& Finkbeiner, D. P. 2011, ApJ, 737, 103

Schlegel, D. J., Finkbeiner, D. P., \& Davis, M. 1998, ApJ, 500, 525

Schmeja, S., Kharchenko, N. V., Piskunov, A. E., et al. 2014, A\&A, 568, A51

Scott, D. W. 1979, Biometrika, 66, 605

Scowcroft, V., Freedman, W. L., Madore, B. F., et al. 2016, ApJ, 816, 49

Silverman, B. 1986, Density Estimation for Statistics and Data Analysis, Chapman \& Hall/CRC Monographs on Statistics \& Applied Probability (Taylor \& Francis)
Simonoff, J. S., \& Udina, F. 1997, Computat. Statist. Data Anal., 23, 335 Small, E. E., Bersier, D., \& Salaris, M. 2013, MNRAS, 428, 763

Sollima, A., Carballo-Bello, J. A., Beccari, G., et al. 2010, MNRAS, 401, 577 Subramanian, S., \& Subramaniam, A. 2009, A\&A, 496, 399

Tammann, G. A., Sandage, A., \& Reindl, B. 2003, A\&A, 404, 423

Taylor, M. B. 2005, in Astronomical Data Analysis Software and Systems XIV, eds. P. Shopbell, M. Britton, \& R. Ebert, ASP Conf. Ser., 347, 29

Tsujimoto, T., \& Bekki, K. 2009, ApJ, 700, L69

Udalski, A., Soszynski, I., Szymanski, M., et al. 1999, Acta Astron., 49, 223

Van Der Walt, S., Colbert, S. C., \& Varoquaux, G. 2011, Comp. Sci. Eng., 13, 22

van Rossum, G. 1995, Python tutorial, Report CS-R9526, pub-CWI:adr

Vanderplas, J., Connolly, A., Ivezić, Ž., \& Gray, A. 2012, in Conference on Intelligent Data Understanding (CIDU), 47

von Hippel, T. 2005, ApJ, 622, 565

von Hippel, T., van Dyk, D. A., Stenning, D. C., et al. 2014, in EAS Pub. Ser., 65,267 


\section{Appendix A: Total cluster mass validation}

The likelihood used in this work (Eq. (1)) allows us to set the total cluster mass as a free parameter to be optimized. To validate ASteCA's mass recovery we processed 768 synthetic clusters generated with the MASSCLEAN tool, 384 for each Magellanic Cloud. These clusters imitate the metallicity and age range for clusters in both Clouds with a large maximum mass. The distance and reddening parameters were fixed; see Table A.1. The process of generating a MASSCLEAN cluster was described in Paper I, Sect. 3. These clusters are only affected by stellar crowding in their faintest magnitudes (using a theoretical completeness function similar to that presented in Small et al. 2013). As such, their mass estimations do not suffer from the systematic underestimation seen in Sect. 5.2.1. Each MASSCLEAN cluster had its $V$ versus $(B-V)$ CMD (in the $U B R I J H K$ photometric system) analyzed by ASteCA.

In Fig. A.1 we show the masses recovered for the 768 MASSCLEAN clusters. The $x$-axis displays the true MASSCLEAN mass values. The y-axis shows the logarithmic mass differences, in the sense ASteCA minus MASSCLEAN. Colors follow the differences in $\log ($ age/yr) (ASteCA minus MASSCLEAN), shown in the right plot color bar. Average age differences for each mass region are $-0.3 \pm 0.6 \operatorname{dex}\left(\bar{M} \leq 1000\left[M_{\odot}\right]\right)$, $-0.05 \pm 0.19 \operatorname{dex}\left(1000<\bar{M} \leq 10000\left[M_{\odot}\right]\right)$, and $-0.01 \pm$ $0.13 \operatorname{dex}\left(\bar{M}>10000\left[M_{\odot}\right]\right)$. As expected, clusters with higher masses have their ages more accurately recovered. On average, the difference between ASteCA (estimated) minus MASSCLEAN (true) logarithmic ages in the full mass range is $\sim-0.1 \pm$ 0.4 dex. Gray bands represent the mean and standard deviation for the logarithmic mass differences, $\overline{\Delta M_{\log }}$. For low-mass clusters $-500 M_{\odot}$ or $1000 M_{\odot}$ - the code assigns masses in a range between $\sim 200-3000 M_{\odot}$. In this region ASteCA underestimates the masses of clusters by $\sim 200 M_{\odot}$. This effect is tied to an improper age estimation, where ASteCA incorrectly assigns younger ages to scarcely populated clusters and compensates for the low number of stars by decreasing the total mass. Such an issue is not unexpected for very low-mass clusters.

Table A.2 shows the correlation matrix between the five cluster parameters. We see the usual correlations appear (agemetallicity, metallicity-distance, age-reddening, etc.), as found in Paper I (Table 3). Total mass shows a small positive correlation with the distance modulus. When distance is overestimated, the matched synthetic CMD contains fewer low-mass stars due to the magnitude limit. The likelihood compensates for this loss by increasing its mass.

\section{A.1. Metallicity estimation for different mass values}

The metallicity $(z)$ estimated for the MASSCLEAN set is shown in Fig. A.2, where tendencies are visible. First, as the mass of the cluster grows so does the accuracy of the metallicity estimates. The average difference between true and estimated values remains close to $|\overline{\Delta z}| \approx 0.001$ for the entire mass range, which is expected as $z=0.001$ is the step used by ASteCA. However its standard deviation drops from $\sim 0.01$ to 0.004 for the more massive clusters. Most of the poorest solutions obtained by ASteCA - those with $|\Delta z|>0.01 \mathrm{dex}$ - are associated with low-mass scarcely populated clusters with $\sim 40$ true member stars on average (from two up to a hundred) in their analyzed CMDs. This poor solutions set is composed of 91 clusters $\sim 12 \%$ of the sample -58 of which have $M \leq 1000 M_{\odot}$. Of these 58 low-mass clusters, 38 are assigned younger ages by the code due to an improper field star decontamination process (an expected issue when the number of true members are very low). Of the 82 clusters with the worst age estimates by ASteCA $\mid \Delta \log ($ age $/ \mathrm{yr}) \mid \geq 0.5 \mathrm{dex}, \sim 11 \%$ of the MASSCLEAN sample $\sim 90 \%$ (73) are clusters with $M \leq 1000 M_{\odot}$. Leaving out these 82 clusters, the average difference in $z$ for the entire mass range is $\sim 0.0008 \pm 0.006$ dex, which is a rather small difference with reasonable dispersion.

The second tendency is the overestimation of $z$ for the lowest metallicities and its underestimation for the largest metallicities. A balanced distribution around $\Delta z=0$ line is mostly seen for abundances in the middle portion of the analyzed range. This trend is more noticeable for lower masses, but can be found for all mass values. This is a statistical artifact that arises due to the necessarily limited metallicity range analyzed. For clusters with the lowest metal contents $(z=0.001)$, ASteCA can only assign equal or larger metallicities since negative $z$ values are not possible. Equivalently, for clusters with the largest abundances $(z=0.03)$ the code can only associate equal or lower metallicities because of the upper $z$ limit used by ASteCA, which is precisely $z=0.03$. This bias could be avoided for large metallicity clusters by increasing the $z$ range upper limit. It cannot be avoided for the lowest metal abundances.

An external source of errors also needs to be taken into account when analyzing ASteCA's metallicity (and age) estimates, for MASSCLEAN clusters. This is the intrinsic difference between Marigo et al. (2008) isochrones, which are used to generate MASSCLEAN clusters, and PARSEC (Bressan et al. 2012) isochrones, which are used by ASteCA to find the optimal fundamental parameters. These differences are a source of error in the matching process that is not straightforward to quantify. The two sets of tracks have non-negligible dissimilarities beyond the turn-off points for most of the age range where they can be produced. This can be seen in Fig. A.3, where isochrones from both sets are compared for five different $\log ($ age/yr) values from 7.5 to 9.5 dex. For ages up to $8 \mathrm{dex}$, PARSEC isochrones present a turn-off point located at lower $\log \left(L / L_{\odot}\right)$ values, particularly for lower metallicities. This causes a shift in the more evolved portions of the isochrone, displacing the Marigo isochrones toward larger $\log \left(L / L_{\odot}\right)$ values. Beyond that age this effects reverses, and PARSEC isochrones are now lifted above the Marigo tracks. Given the many known correlations between fundamental parameters, it is not easy to predict how the matching algorithm will resolve such instances. 
A\&A 602, A89 (2017)

Table A.1. Parameter values used to generate the set of 768 MASSCLEAN clusters.

\begin{tabular}{lcc}
\hline \hline Parameter & Values & $\mathrm{N}$ \\
\hline$z$ & $0.001,0.004,0.015,0.03$ & 4 \\
$\log (\mathrm{age} / \mathrm{yr})$ & $18.9(\mathrm{SMC}), 18.5(\mathrm{LMC})$ & 12 \\
$\mu_{0}$ & 0.1 & 2 \\
$E_{B-V}$ & $5,7.2,7.5,7.7,8,8.2,8.5,8,9,9.2,9.5,9.7$ & 1 \\
Mass $\left(M_{\odot}\right)$ & $500,1000,5000,10000,25000,50000,100000,250000$ & 8 \\
\hline
\end{tabular}

Table A.2. Correlation matrix for parameter deltas, defined for each cluster in the sense ASteCA minus MASSCLEAN.

\begin{tabular}{lccccc}
\hline \hline$\Delta$ param & $\Delta z$ & $\Delta \log (\mathrm{age} / \mathrm{yr})$ & $\Delta \mu$ & $\Delta E_{B-V}$ & $\Delta M$ \\
\hline$\Delta z$ & 1. & -0.36 & 0.24 & -0.15 & 0.03 \\
$\Delta \log (\mathrm{age} / \mathrm{yr})$ & - & 1. & -0.15 & -0.28 & 0.01 \\
$\Delta \mu$ & - & - & 1. & 0.05 & 0.13 \\
$\Delta E_{B-V}$ & - & - & - & 1. & 0.0 \\
$\Delta M$ & - & - & - & - & 1. \\
\hline
\end{tabular}
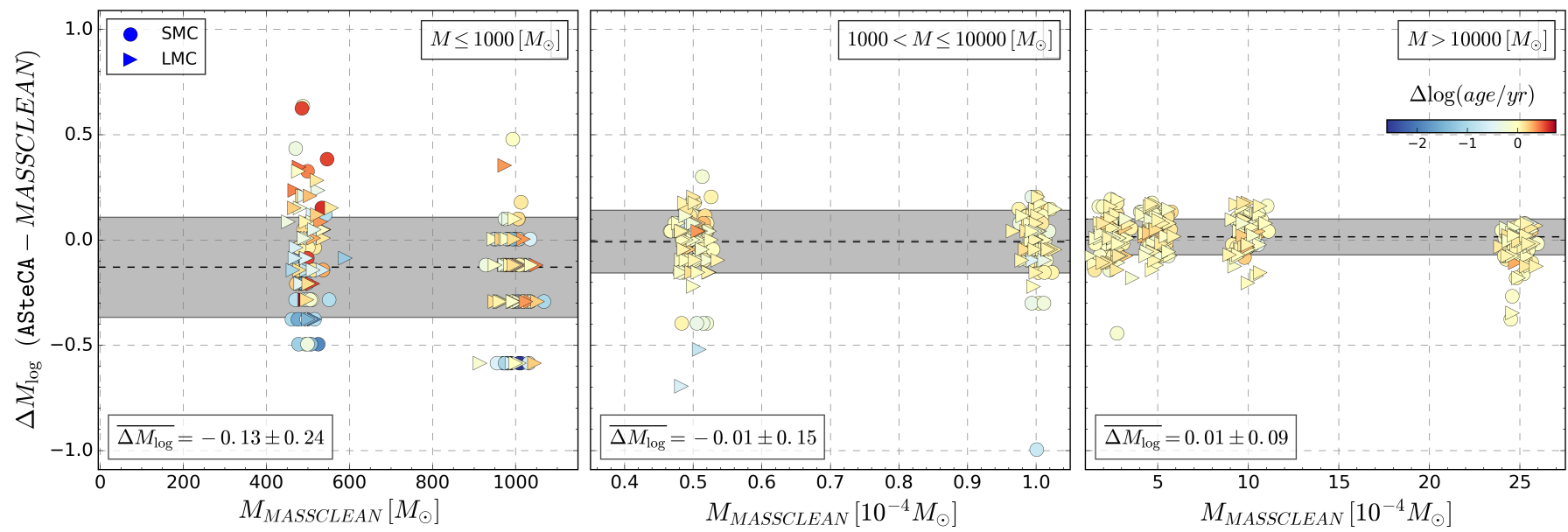

Fig. A.1. Recovered masses by ASteCA for the 768 MASSCLEAN clusters. Logarithmic mass differences $\Delta M_{\text {log }}$ are obtained in the sense ASteCA minus MASSCLEAN and shown in the $y$-axis. MASSCLEAN masses in the $x$-axis are perturbed with a small random scatter.
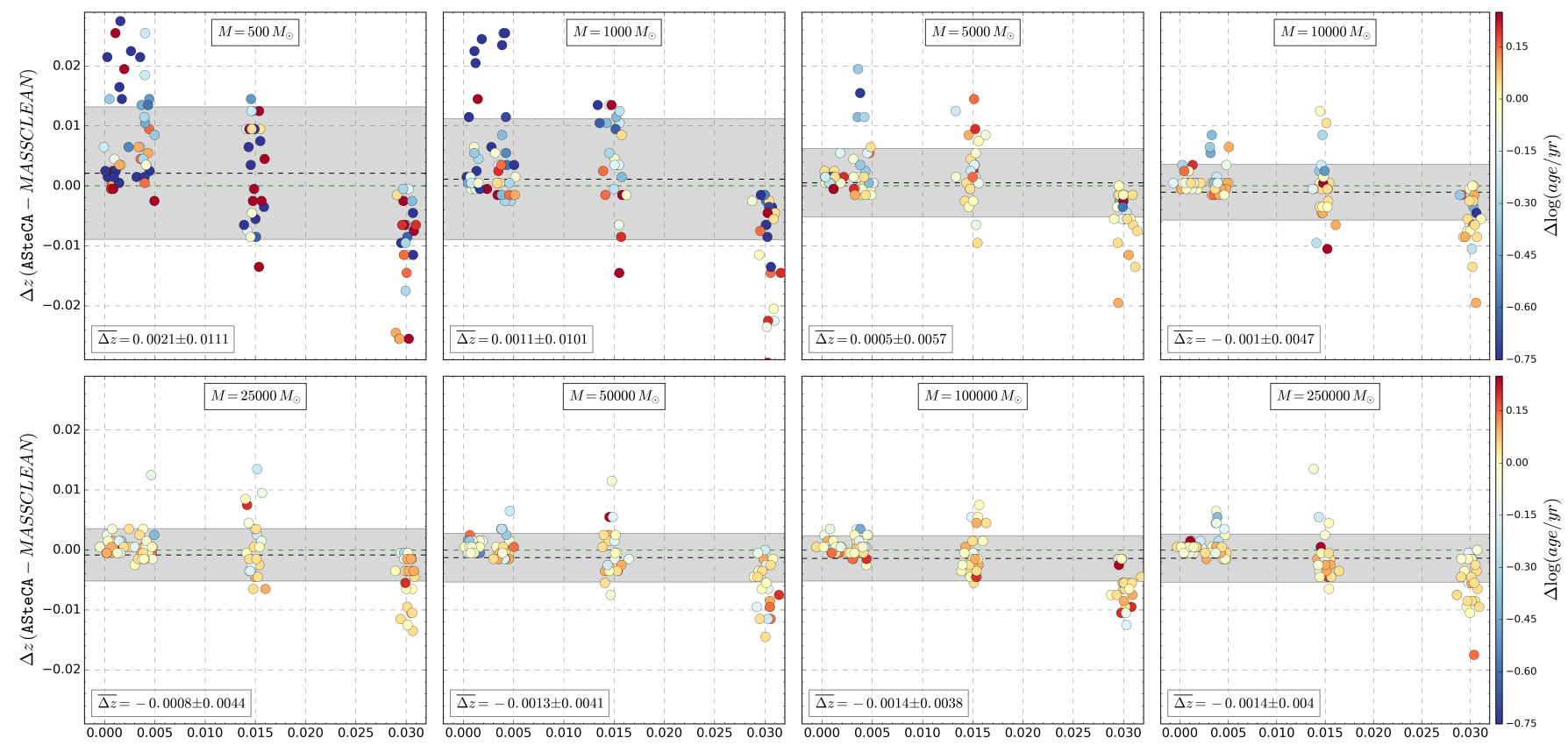

$z_{\text {MASSCLEAN }}$

$z_{\text {MASSCLEAN }}$

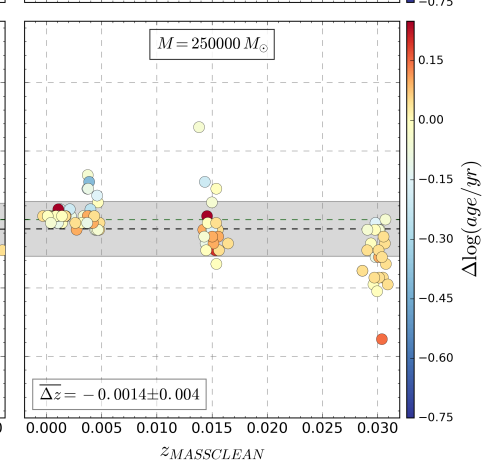

Fig. A.2. ASteCA metallicity estimates for each mass used to generate MASSCLEAN clusters. Colors are associated with log(age/yr) differences, shown in the color bars to the right. The green dashed horizontal line is the $\Delta[\mathrm{Fe} / \mathrm{H}]=0$ line, shown as reference. 
G. I. Perren et al.: Astrophysical properties of star clusters in the Magellanic Clouds homogeneously estimated by ASteCA
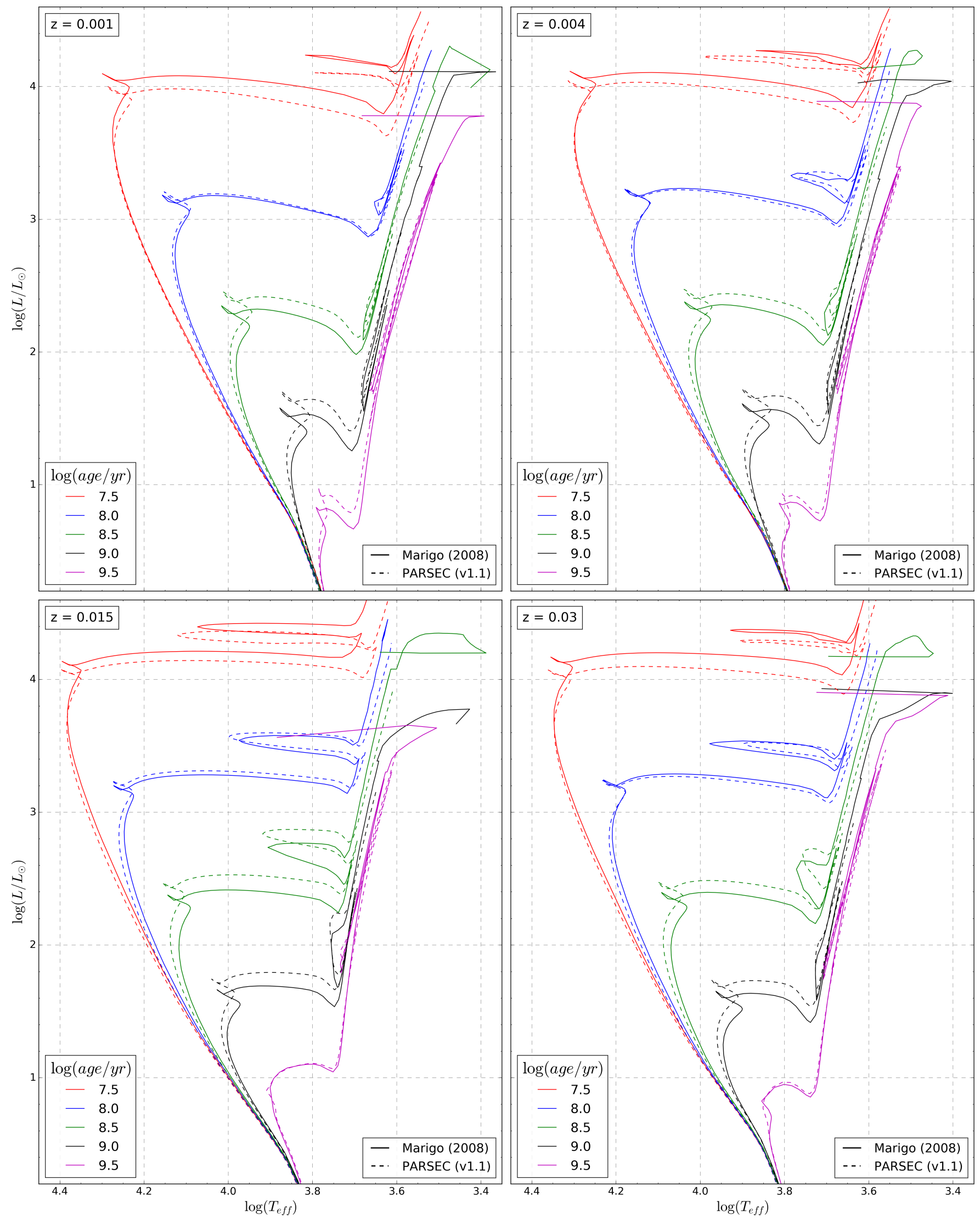

Fig. A.3. Marigo et al. (2008) vs. PARSEC (Bressan et al. 2012) isochrones for different metallicities and ages. 


\section{Appendix B: Outliers}

Ten of the analyzed clusters in this work - 4\% of the set show age differences with the literature $\Delta \log ($ age $/ y r)>0.5$. Such a large age difference translates into two very dissimilar isochrones fitted to the same coeval star sequence, which makes this subsample of clusters stand out. For these outliers no configuration of the DA plus the employed binning methods could be found, which resulted in synthetic CMD matches with age values close to those found in the literature. All clusters in the outliers sample had smaller ages assigned by the code, compared to the literature; see Table B.1. These differences go from 0.55 dex up to $1.6 \mathrm{dex}$ in the most extreme case of LMC-KMHK975.

In Fig. B.1 CMDs for these clusters are plotted, two per row. Each CMD pair shows the cluster region with the literature isochrone fit (left) and the best-match isochrone found by ASteCA (right). Colors in the right CMD correspond to the MPs assigned by the DA, while semi-transparent stars are those removed by the cell-by-cell density-based cleaning algorithm (see Sect. 3.3).

For most of the outliers, the same process is identified as the main cause responsible for the observed age differences. While the literature by-eye isochrone fit aligns the brighter part of the sequence of the cluster with the turn-off point of an older isochrone, ASteCA decides instead that this is the top portion of a much younger isochrone with no discernible turn off. The statistical mismatch due to the removal of low-mass stars by the DA, which is discussed in Sect. 5.1, can also be seen to affect some of the fits. In particular, SMC clusters SL579 and H86-188 show signs of this effect in the best synthetic CMD match selected by ASteCA (see Fig. B.1, CMDs $b$ and $h$ ).
Table B.1. Clusters with large age differences between the literature and values found by the code (outliers).

\begin{tabular}{lccccc}
\hline \hline Cluster & $\alpha\left(^{\circ}\right)$ & $\delta\left(^{\circ}\right)$ & $L$ & $A$ & $\Delta$ \\
\hline L-KMHK975 & 82.49583 & -67.87889 & 8.30 & 6.70 & 1.60 \\
L-SL579 & 83.55417 & -67.85639 & 8.15 & 7.00 & 1.15 \\
L-BSDL631 & 76.64167 & -68.42722 & 8.35 & 7.50 & 0.85 \\
L-KMHK979 & 82.41250 & -70.98389 & 7.90 & 7.30 & 0.60 \\
L-H88-316 & 85.41250 & -69.22944 & 8.25 & 7.70 & 0.55 \\
S-L35 & 12.00417 & -73.48611 & 8.34 & 6.90 & 1.44 \\
S-H86-188 & 15.05833 & -72.45833 & 8.10 & 6.70 & 1.40 \\
S-L39 & 12.32500 & -73.37167 & 8.05 & 7.00 & 1.05 \\
S-B134 & 17.25417 & -73.20667 & 8.15 & 7.20 & 0.95 \\
S-K47 & 15.79583 & -72.27361 & 7.90 & 7.00 & 0.90 \\
\hline
\end{tabular}

Notes. Equatorial coordinates are expressed in degrees for the J2000.0 epoch. Ages are given as $\log ($ age/yr) for literature $(L)$ and ASteCA $(A)$. The difference between both estimates $(L-A)$ is given in the last column as $\Delta$.

These age estimates could be brought closer to literature values, if a more restrictive age range was used (e.g., a minimum value of $\log ($ age $/ y r)=7.5$ dex instead of 6 dex as used in this work). Lacking external evidence to substantiate this a priori restriction, we choose to keep the values obtained by ASteCA, with this section acting as a cautionary note. 
G. I. Perren et al.: Astrophysical properties of star clusters in the Magellanic Clouds homogeneously estimated by ASteCA
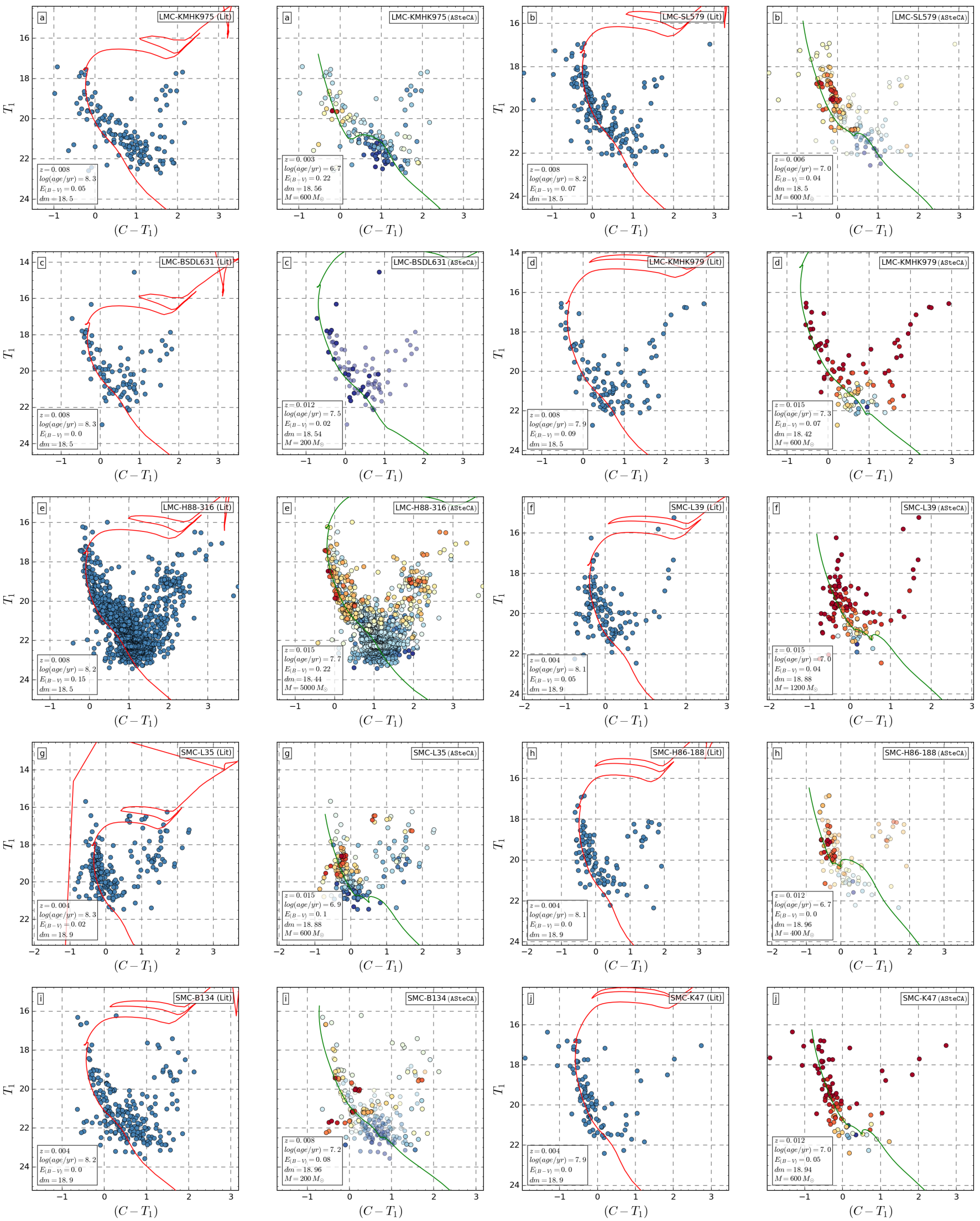

Fig. B.1. CMDs for the outliers set. Description of the plots in the main text of the section. 


\section{Appendix C: Color-magnitude diagrams} for the P99, P00, C06, and G10 databases

Figures C.1 to C.18 present the CMDs of clusters cross-matched with our own sample in the databases P99, P00, C06, and G10, i.e., those that used the isochrone fit method in their analysis. Same data distribution in the plotted CMDs as that described for Fig. B.1.
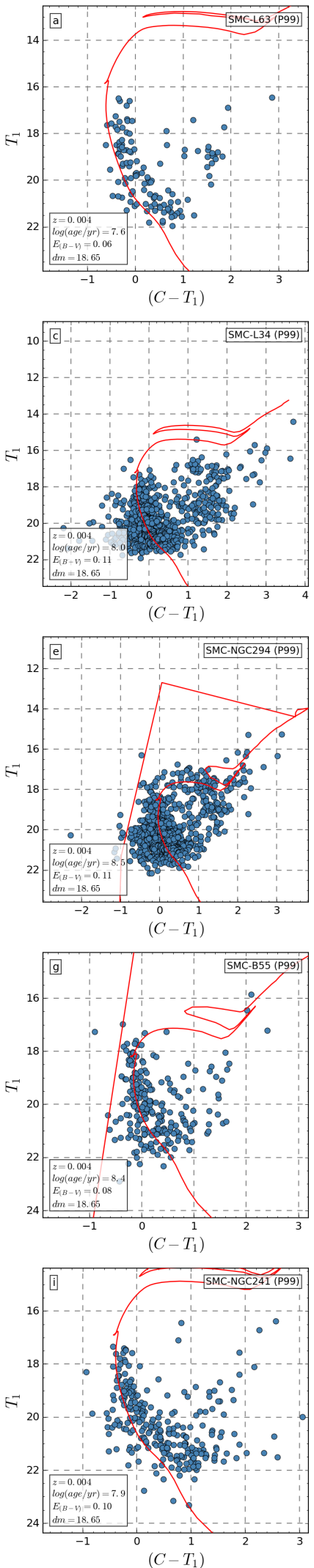

Fig. C.1. CMDs for the P99 database.
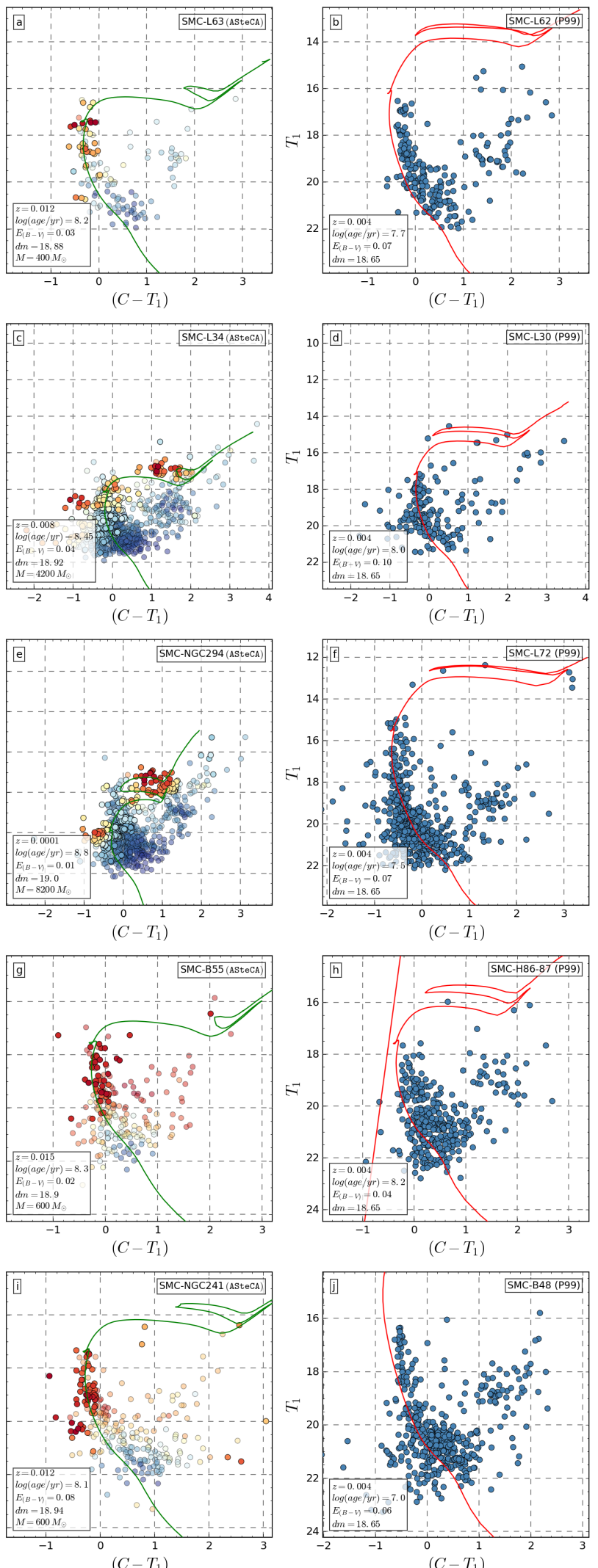
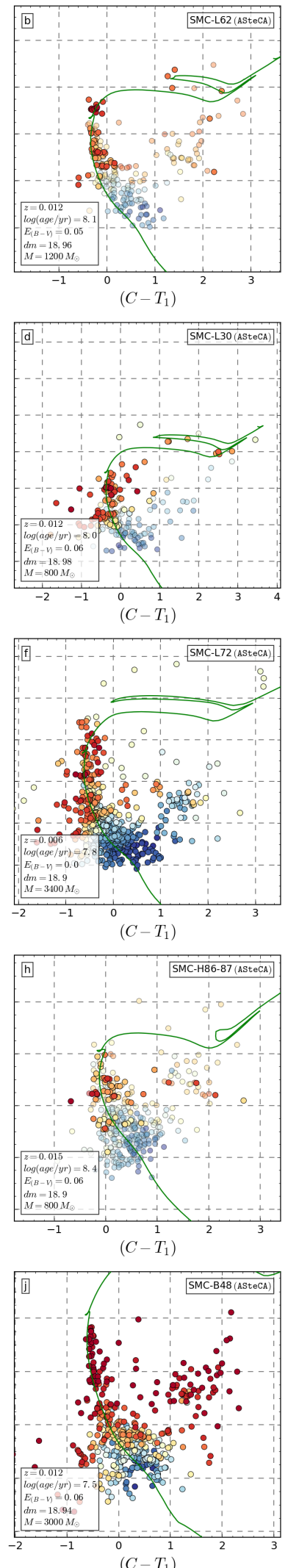
G. I. Perren et al.: Astrophysical properties of star clusters in the Magellanic Clouds homogeneously estimated by ASteCA
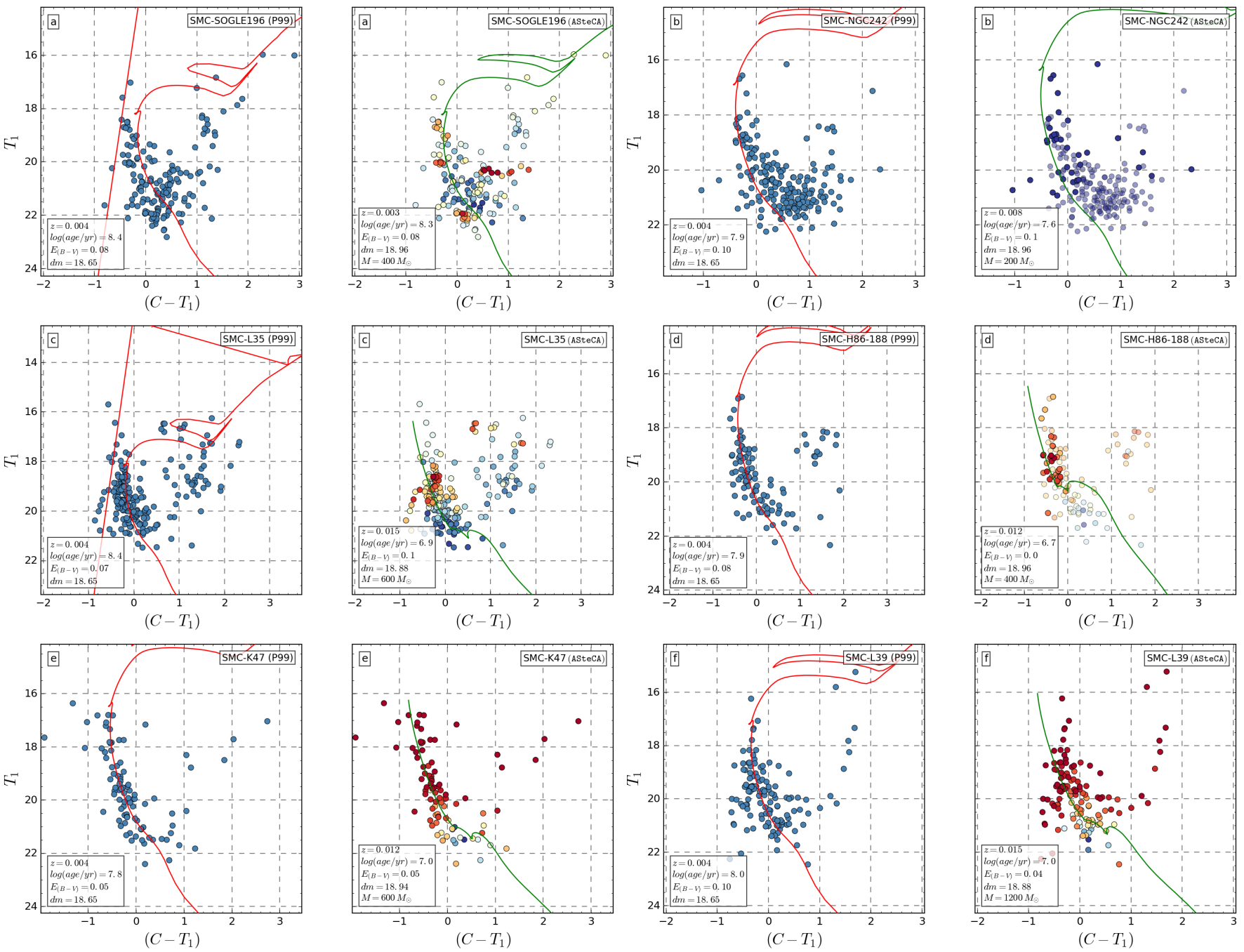

Fig. C.2. CMDs for the P99 database. 
A\&A 602, A89 (2017)
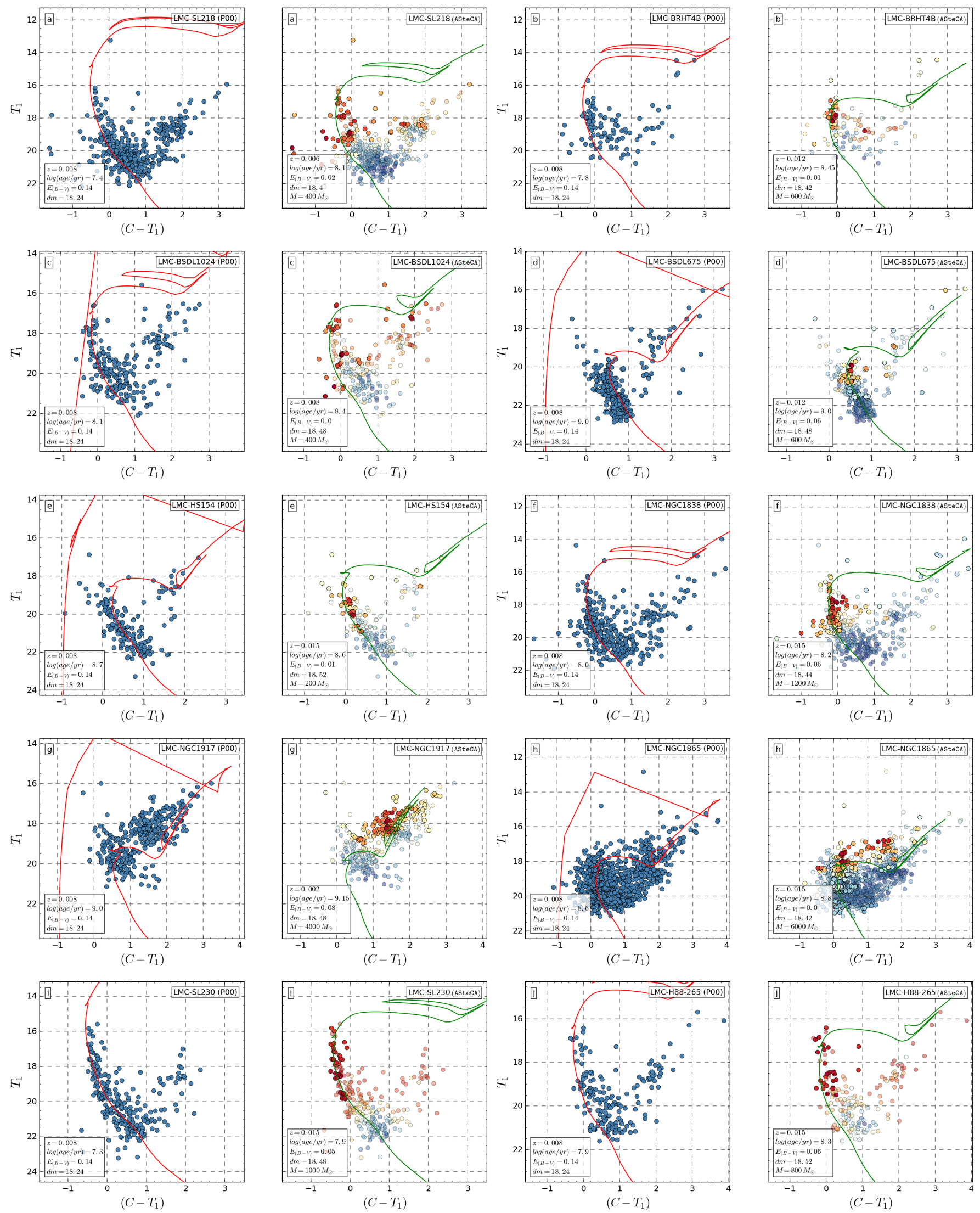

Fig. C.3. CMDs for the P00 database. 
G. I. Perren et al.: Astrophysical properties of star clusters in the Magellanic Clouds homogeneously estimated by ASteCA
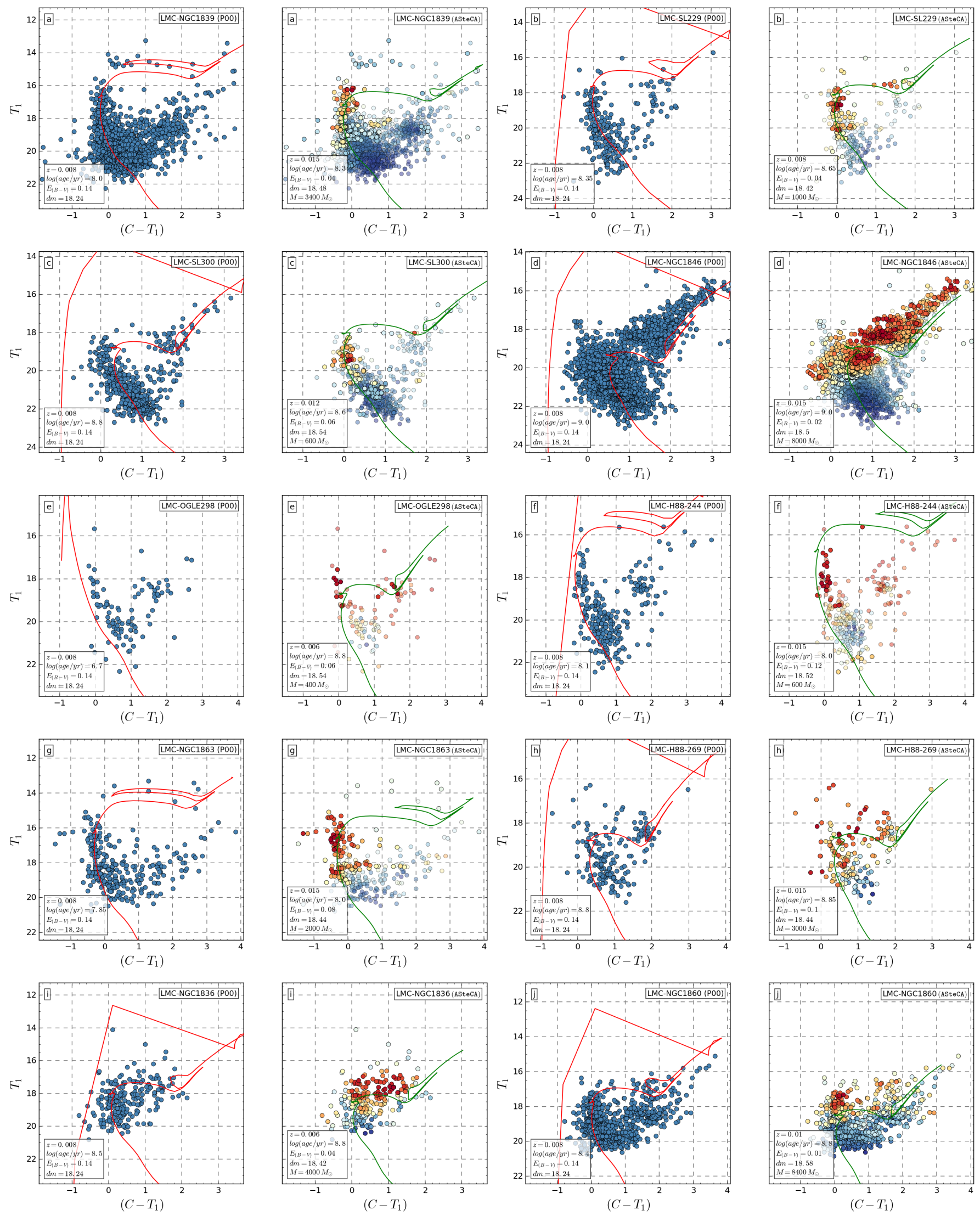

Fig. C.4. CMDs for the P00 database. 
A\&A 602, A89 (2017)
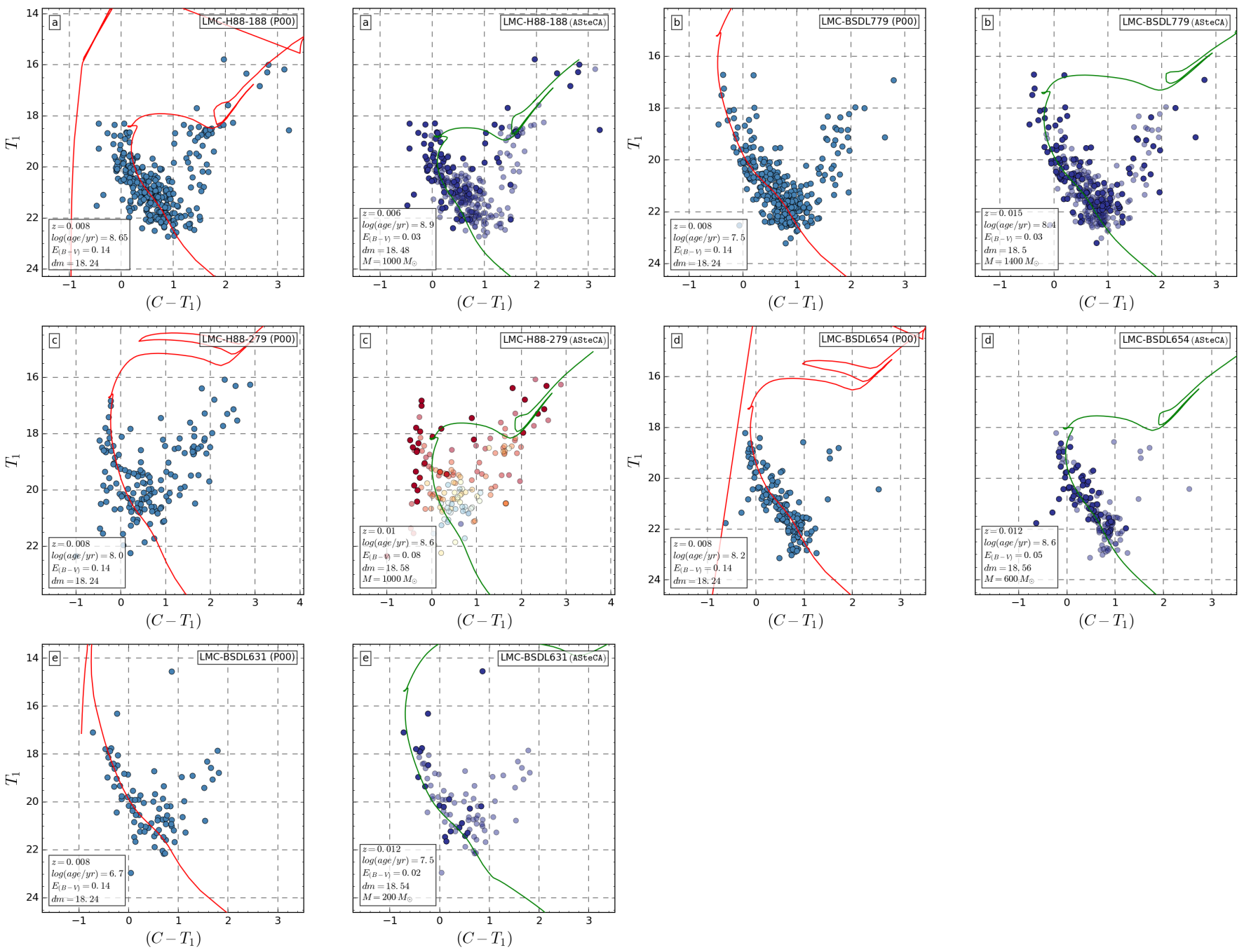

Fig. C.5. CMDs for the P00 database. 
G. I. Perren et al.: Astrophysical properties of star clusters in the Magellanic Clouds homogeneously estimated by ASteCA
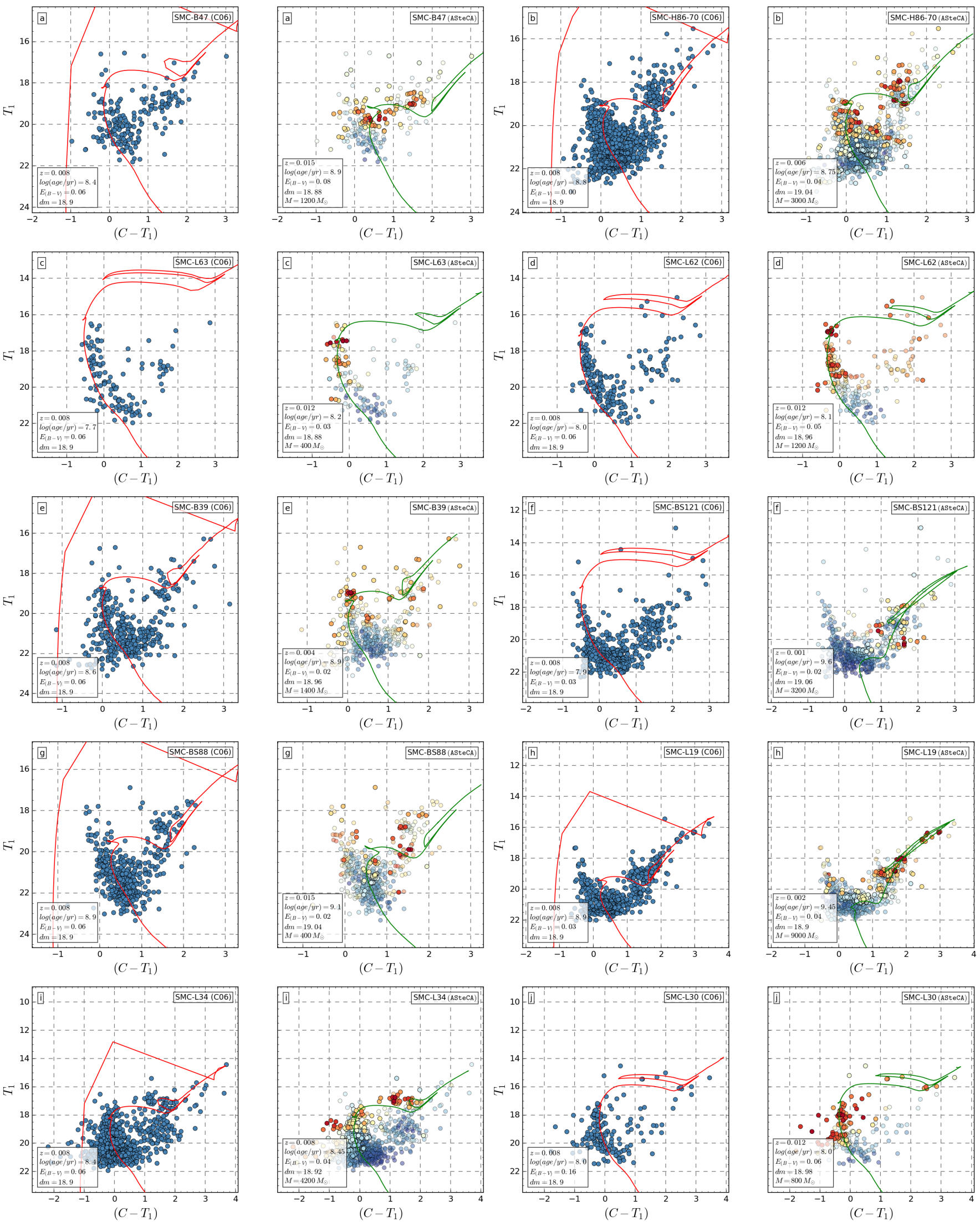

Fig. C.6. CMDs for the C06 database. 
A\&A 602, A89 (2017)
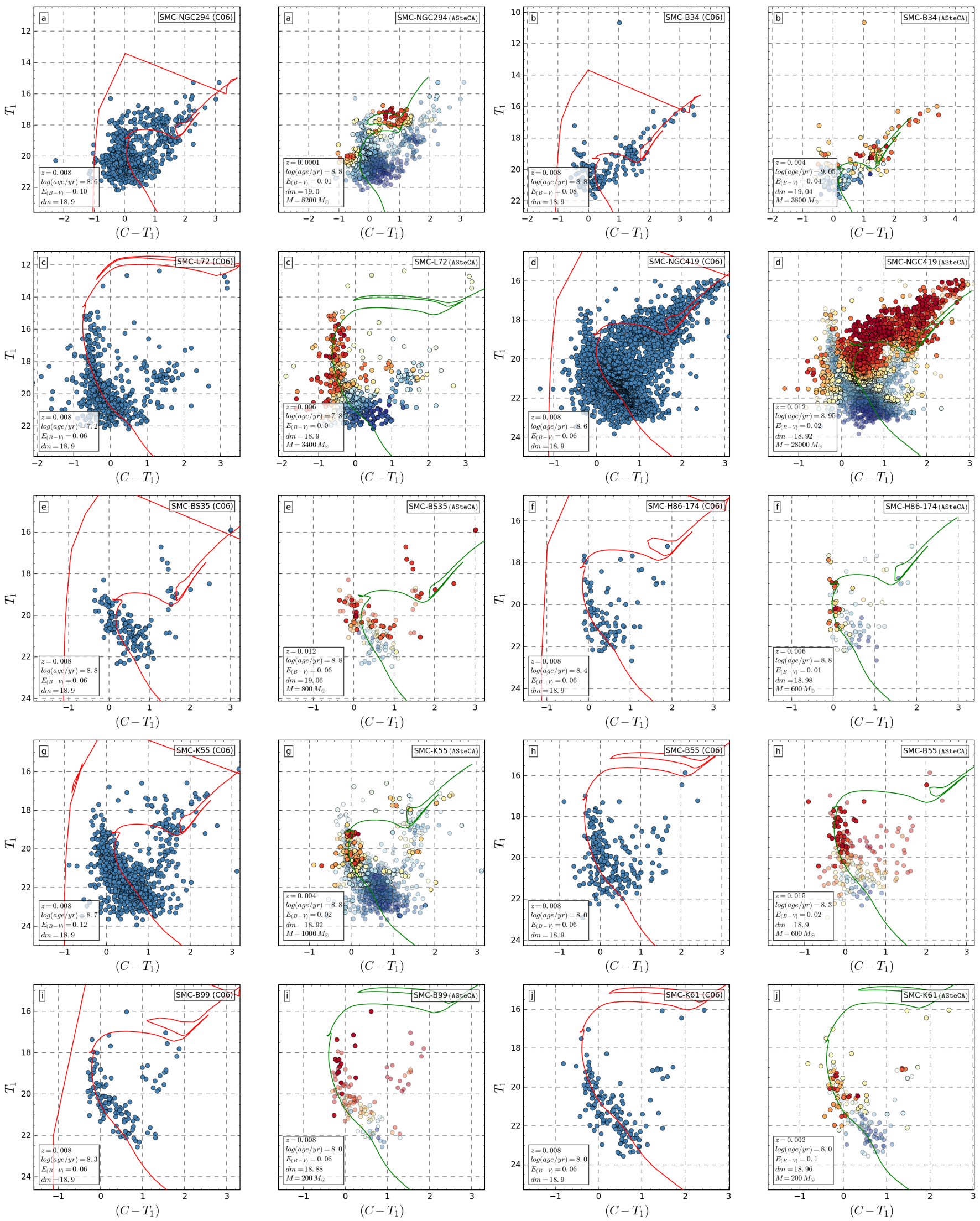

Fig. C.7. CMDs for the C06 database. 
G. I. Perren et al.: Astrophysical properties of star clusters in the Magellanic Clouds homogeneously estimated by ASteCA
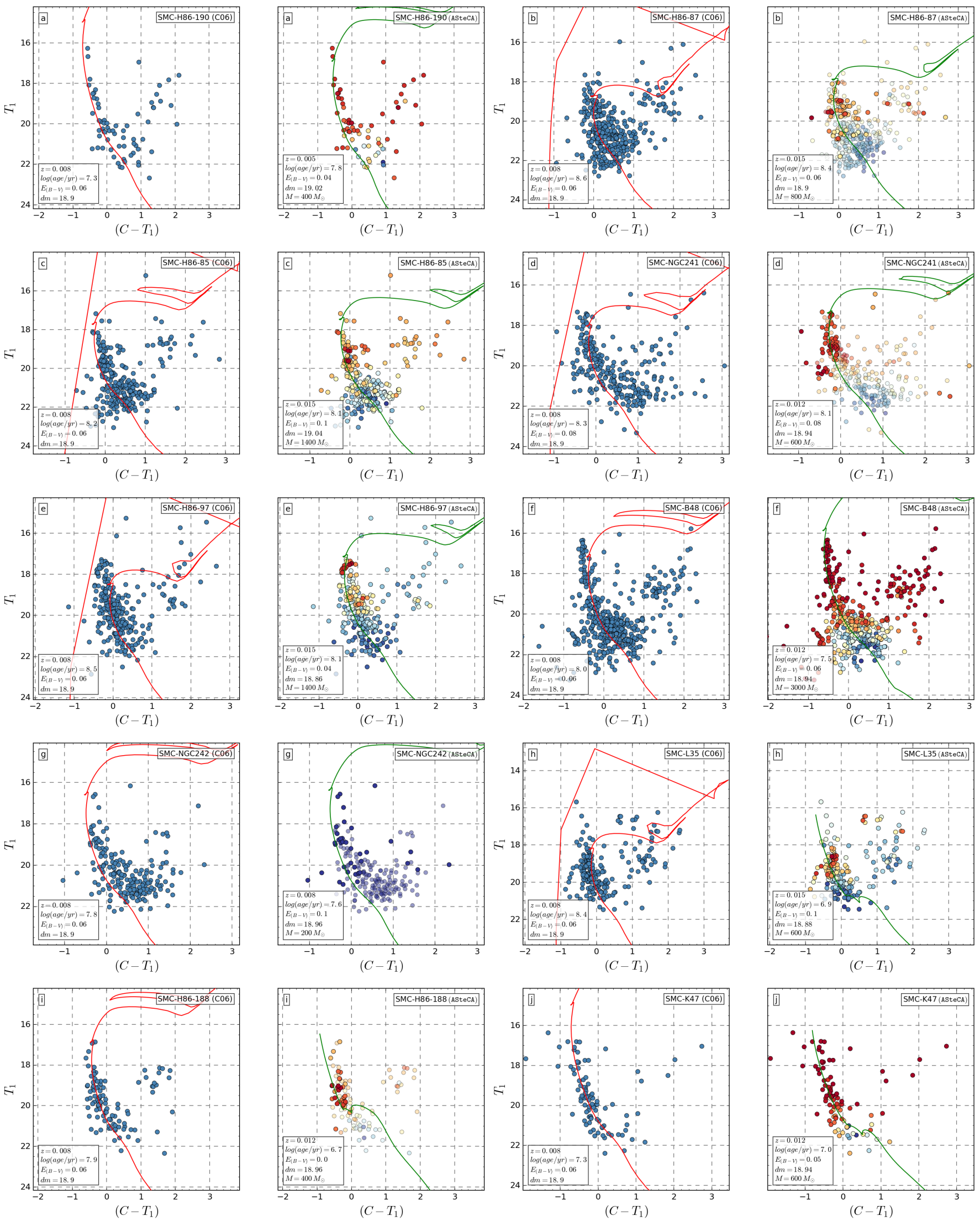

Fig. C.8. CMDs for the C06 database. 
A\&A 602, A89 (2017)
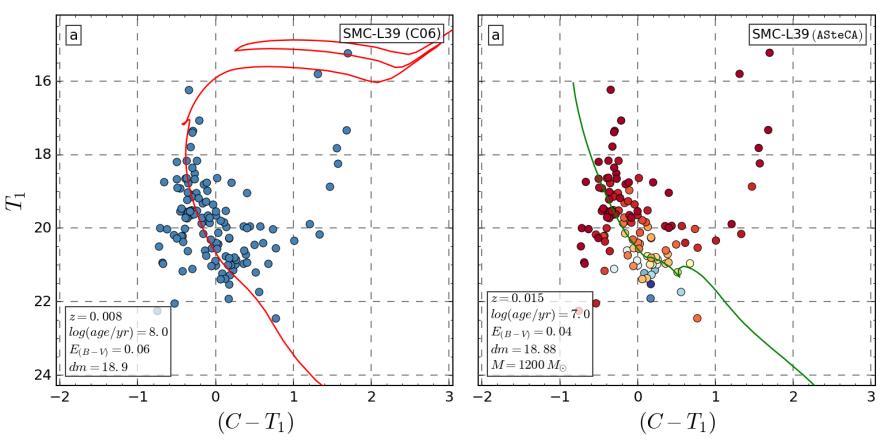

Fig. C.9. CMDs for the C06 database. 
G. I. Perren et al.: Astrophysical properties of star clusters in the Magellanic Clouds homogeneously estimated by ASteCA
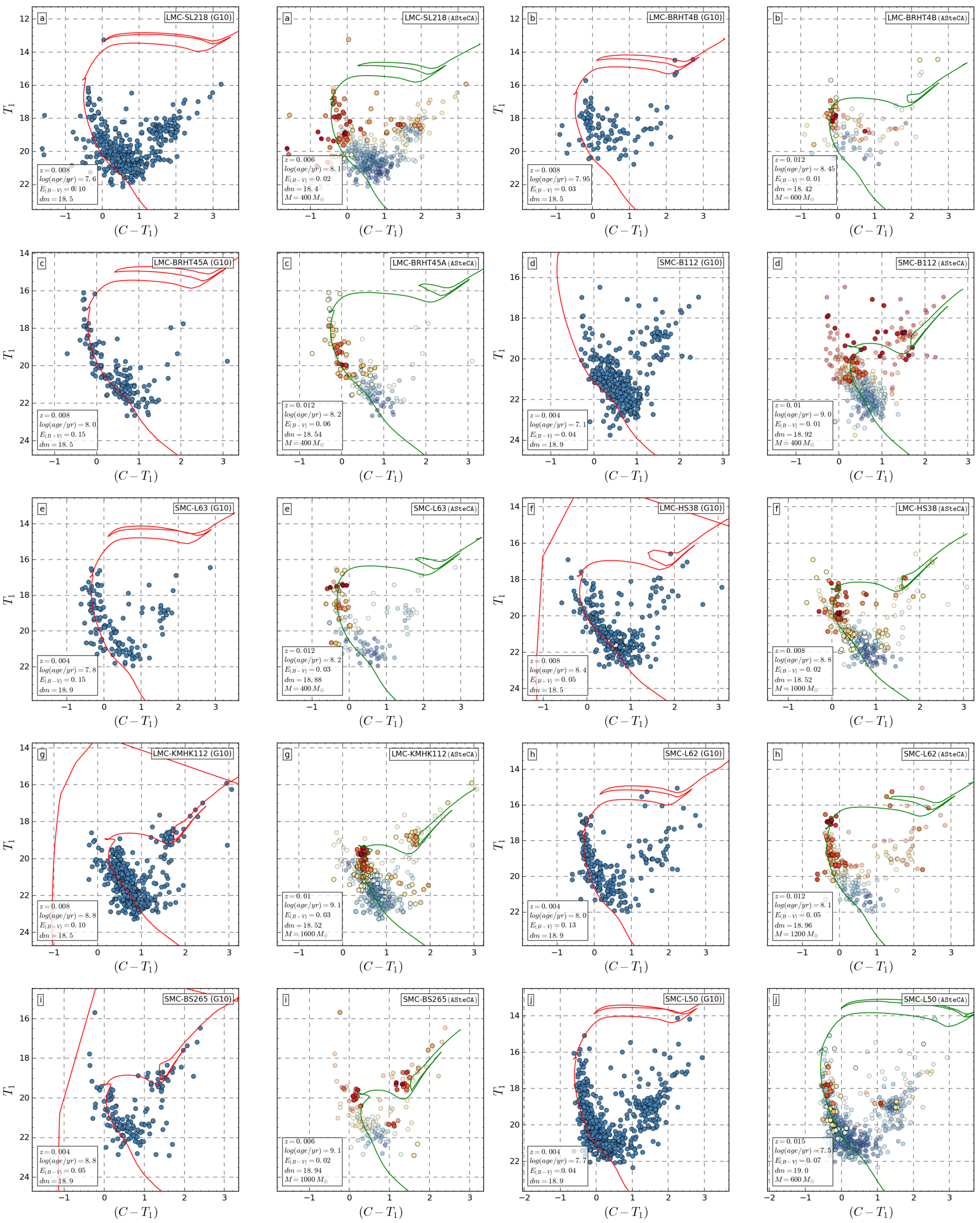

Fig. C.10. CMDs for the G10 database. 
A\&A 602, A89 (2017)
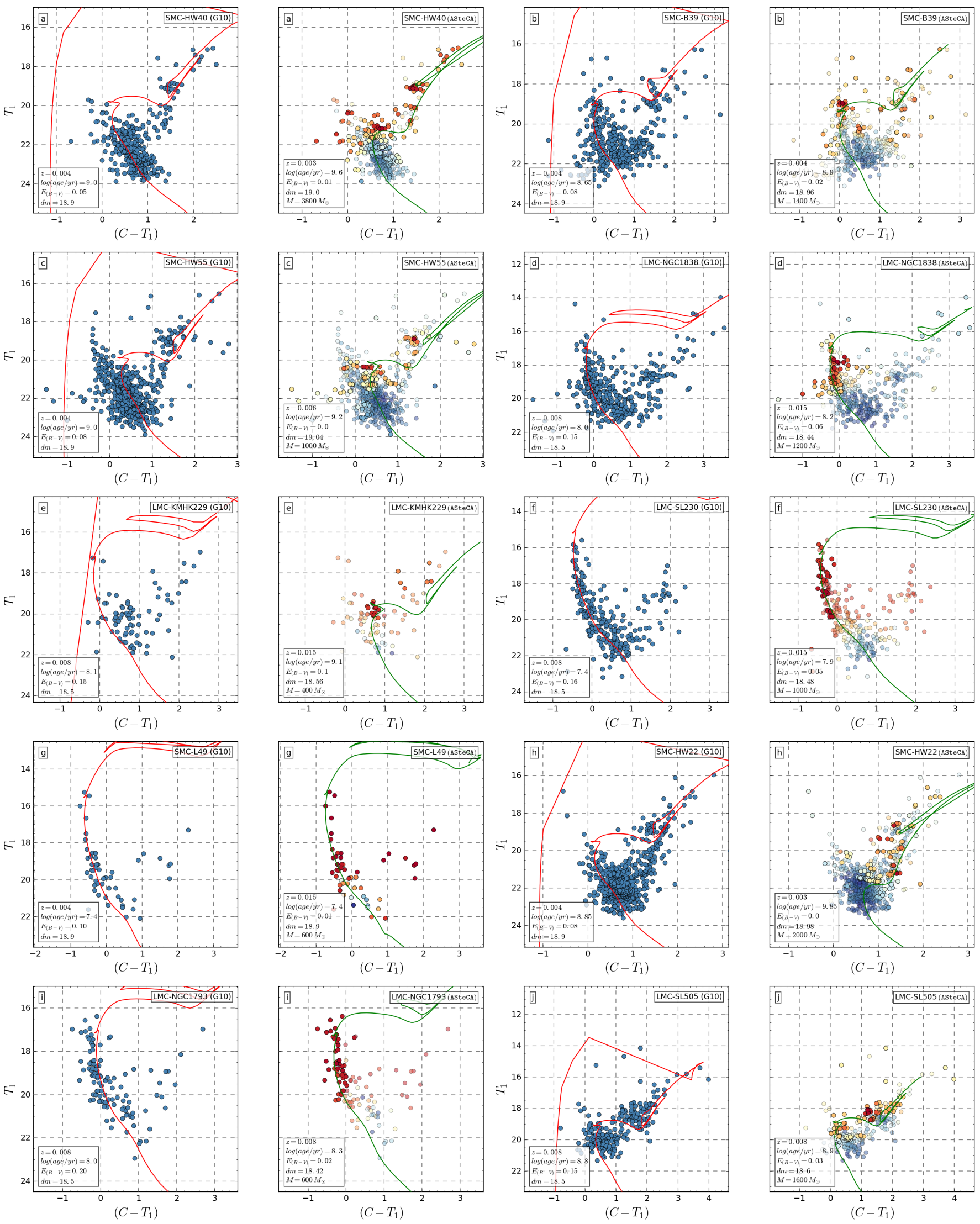

Fig. C.11. CMDs for the G10 database. 
G. I. Perren et al.: Astrophysical properties of star clusters in the Magellanic Clouds homogeneously estimated by ASteCA
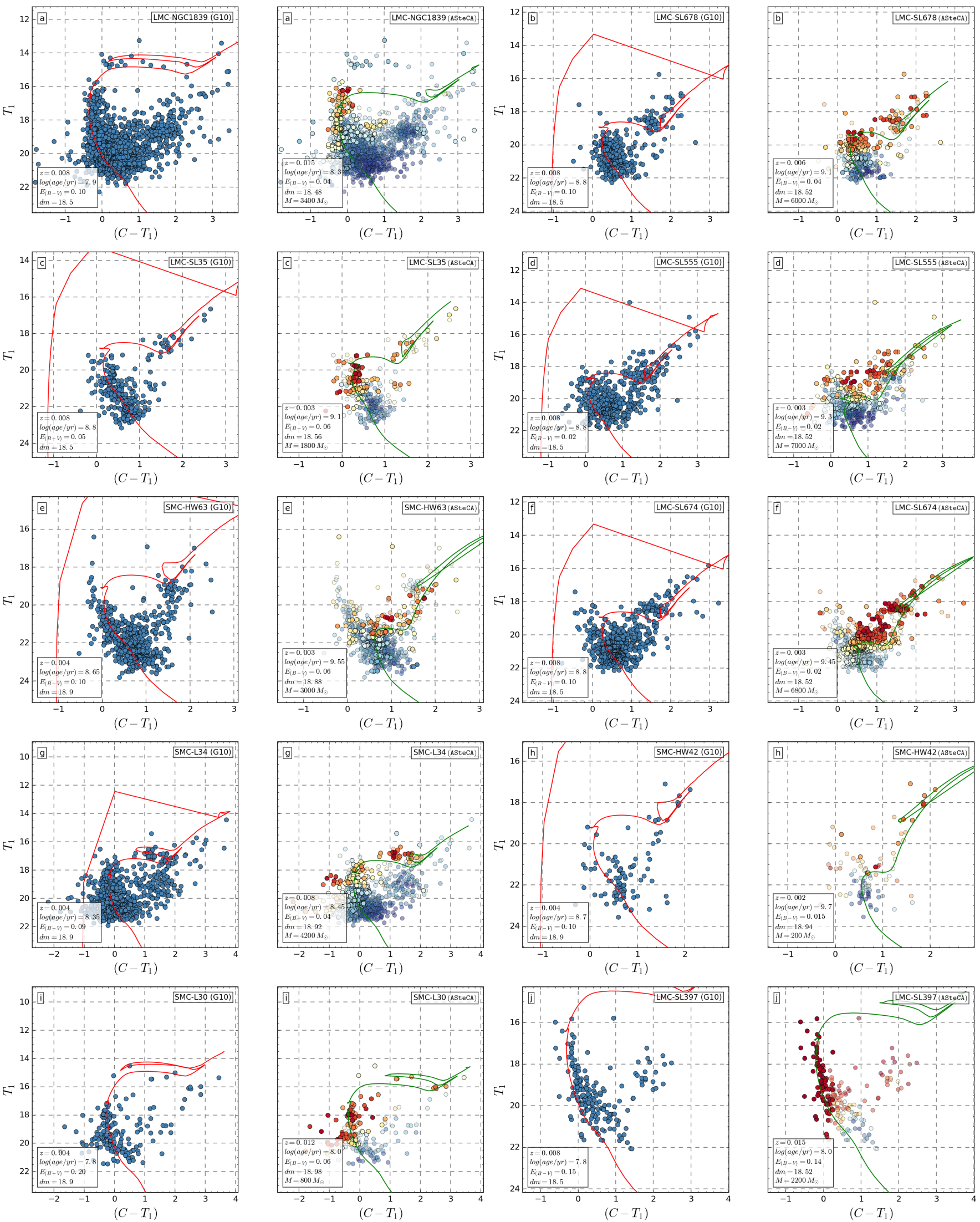

Fig. C.12. CMDs for the G10 database. 
A\&A 602, A89 (2017)
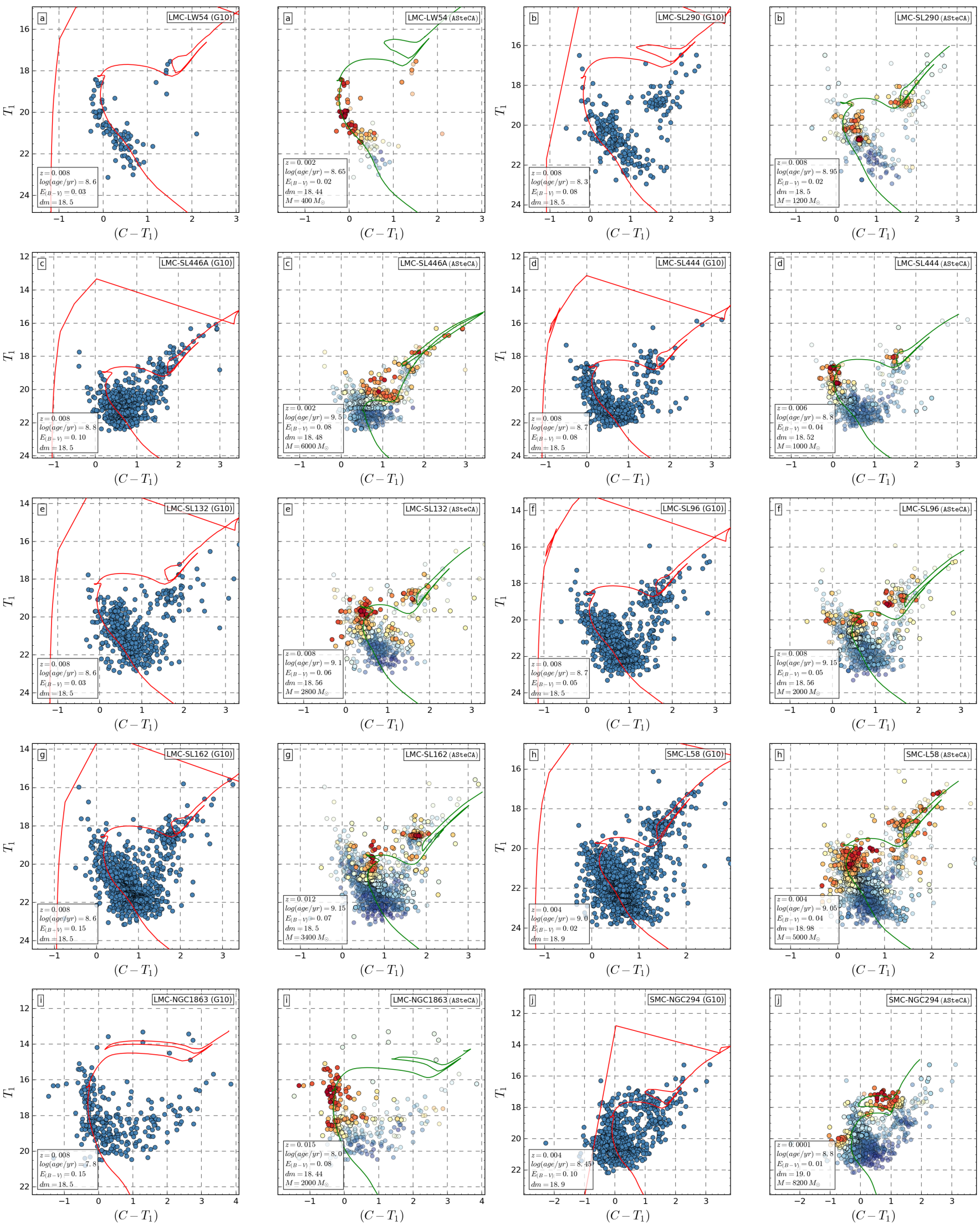

Fig. C.13. CMDs for the G10 database. 
G. I. Perren et al.: Astrophysical properties of star clusters in the Magellanic Clouds homogeneously estimated by ASteCA
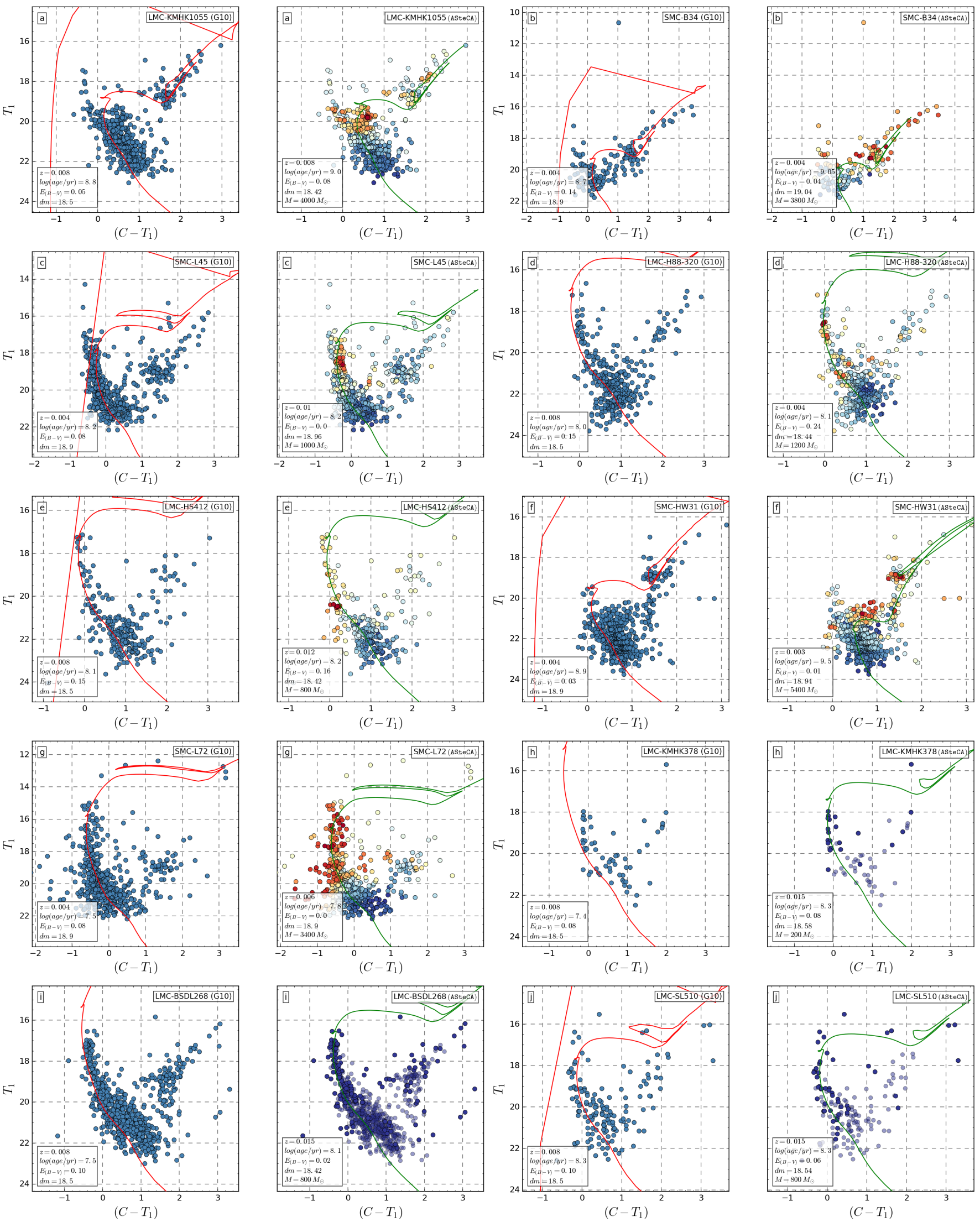

Fig. C.14. CMDs for the G10 database. 

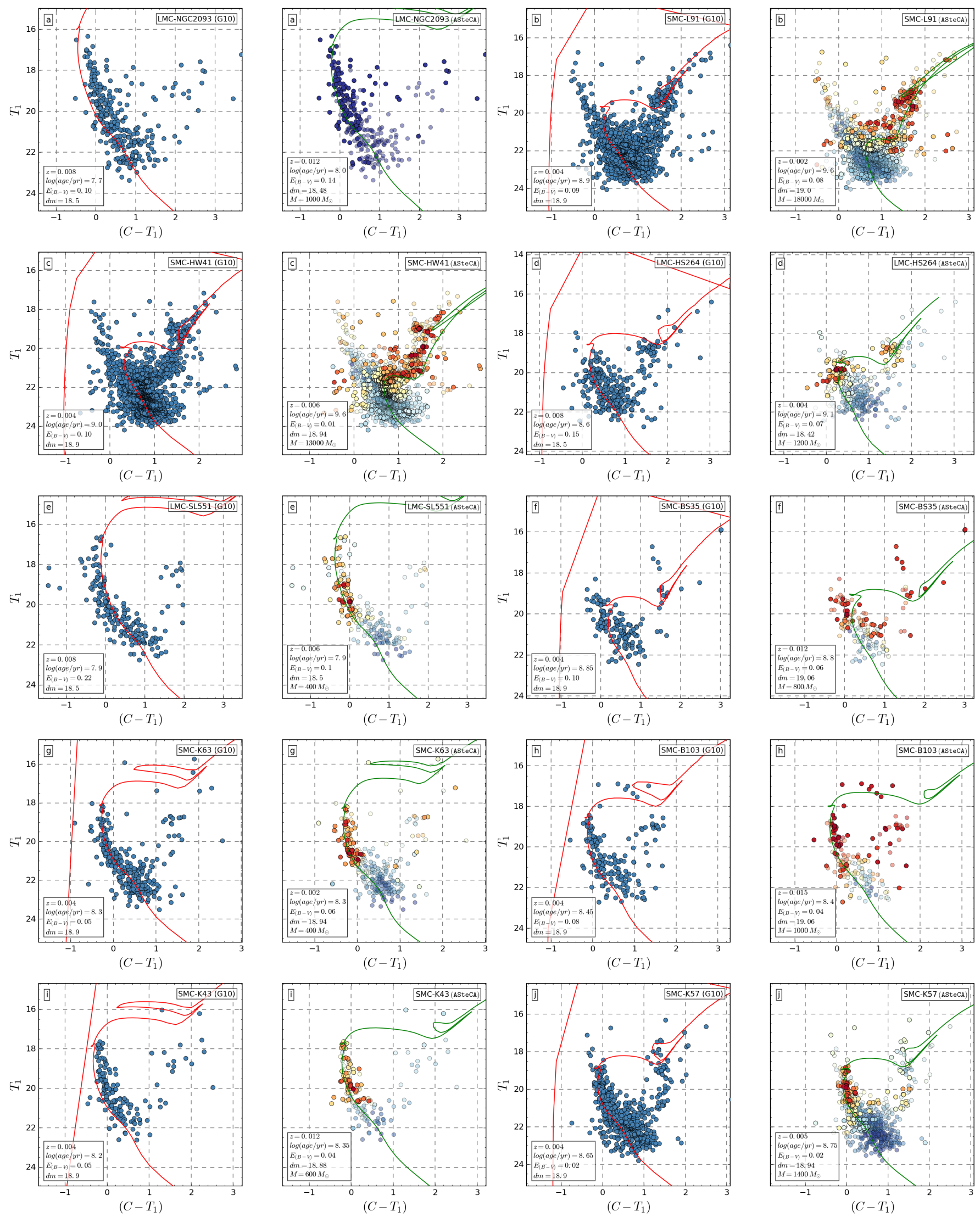

Fig. C.15. CMDs for the G10 database. 
G. I. Perren et al.: Astrophysical properties of star clusters in the Magellanic Clouds homogeneously estimated by ASteCA
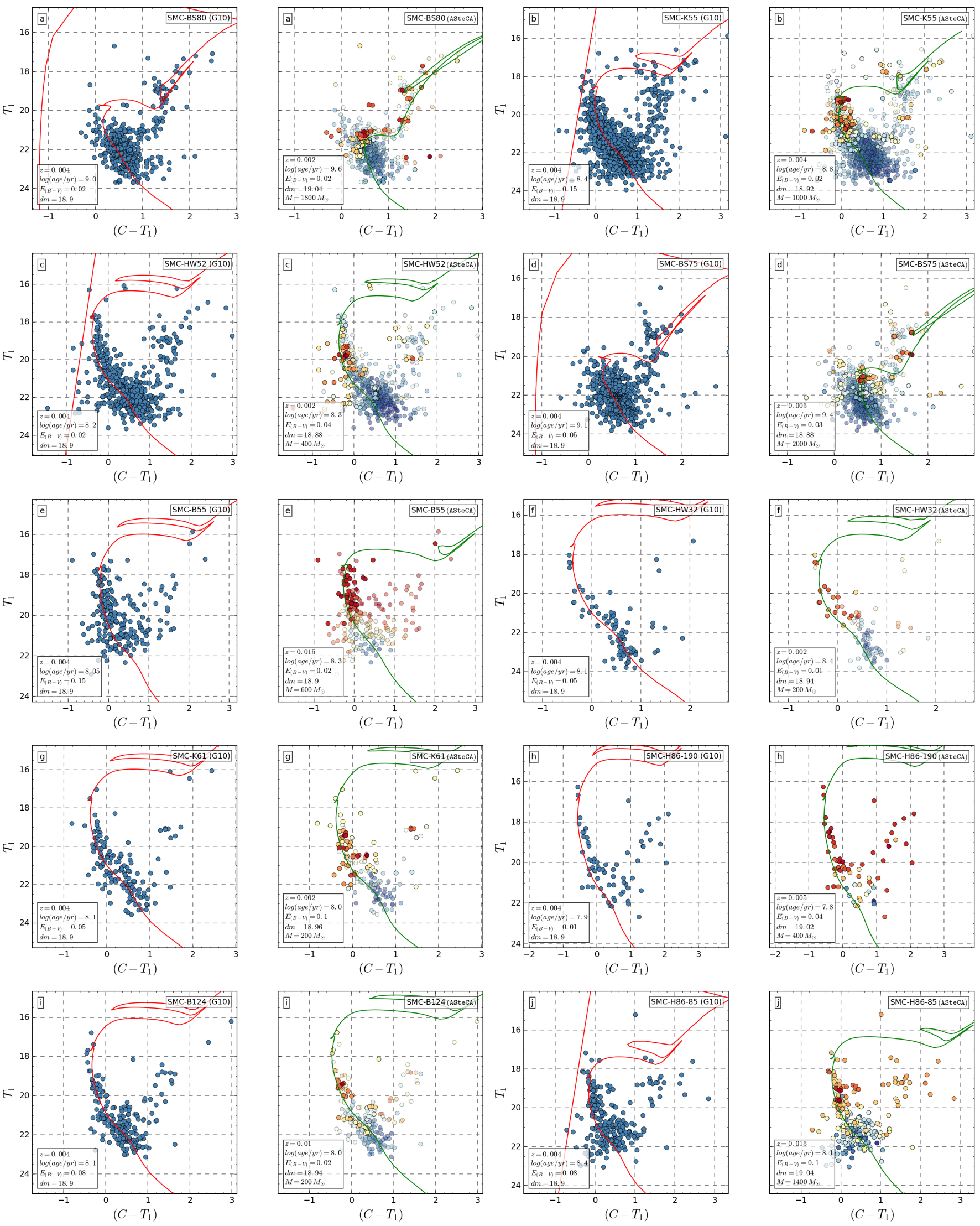

Fig. C.16. CMDs for the G10 database. 
A\&A 602, A89 (2017)
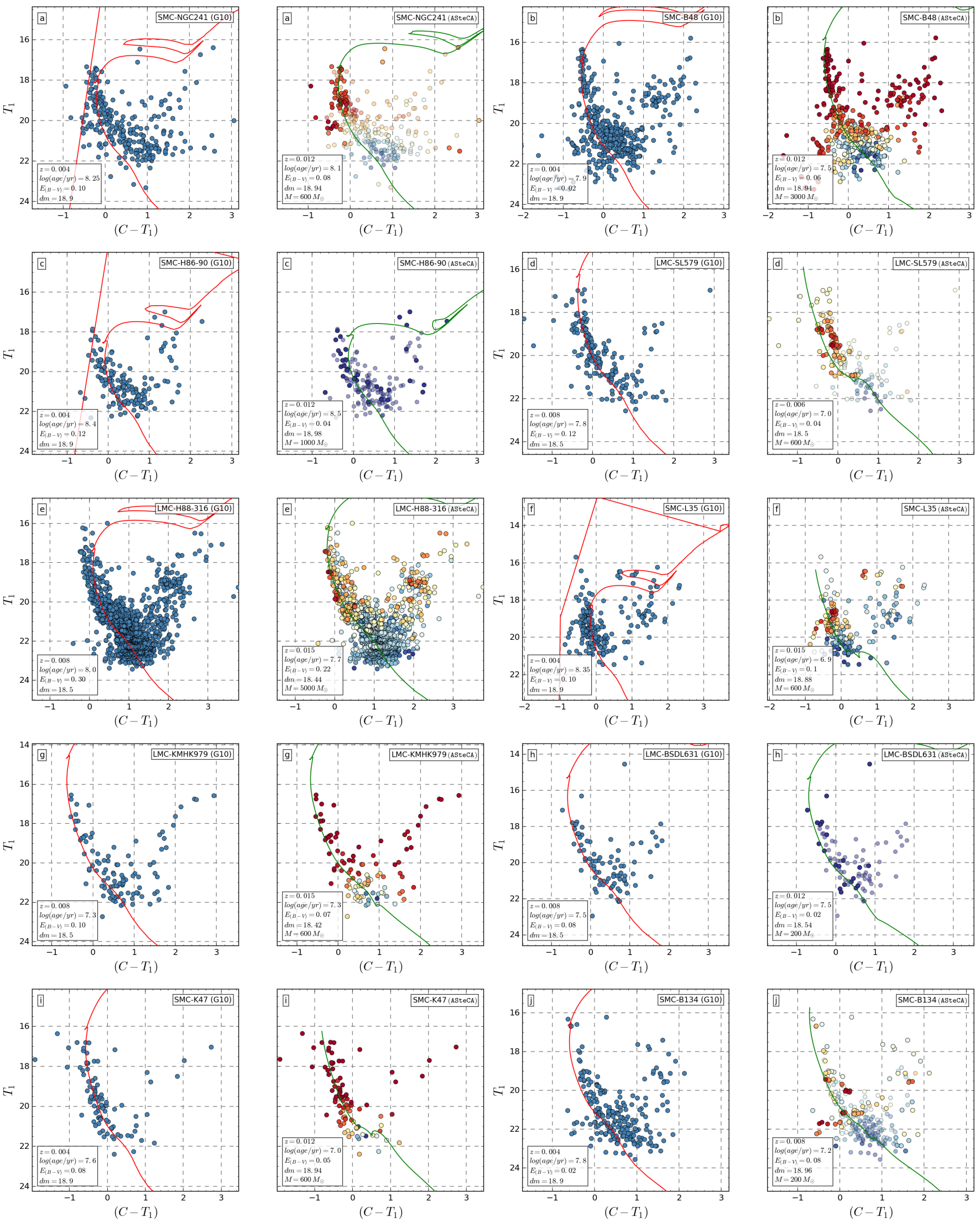

Fig. C.17. CMDs for the G10 database. 

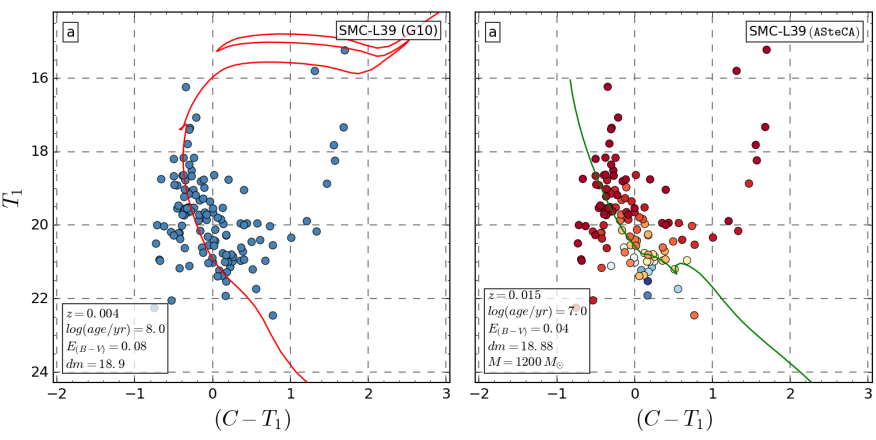

Fig. C.18. CMDs for the G10 database.

\section{Appendix D: Mathematical description of the age-metallicity relationship}

We use the KDE technique described in Sect. 6 to generate an AMR representative of the observed data with some important improvements over previous methods. First, unlike a regular histogram, a Gaussian density map has no dependence on the number, size (fixed or variable), or location of bins. Second, the errors in the two parameters used to obtain the density map (age and metallicity) are organically included in the function that generates it (as explained in Sect. 6). No ad hoc procedure is needed to incorporate the information carried by these values into the final AMR.

The process of creating an AMR function requires assigning a unique $[\mathrm{Fe} / \mathrm{H}]$ to a single age value for the available age range. A dense grid is created to divide the age-metallicity 2D density map into $\mathrm{N}$ steps of 0.01 dex width, covering the ranges of both parameters. Every point in this grid is evaluated in the KDE map and its value $\left(w_{i}\right)$ is stored along with its age-metallicity coordinates $\left(\mathrm{age}_{i},[\mathrm{Fe} / \mathrm{H}]_{i}\right)$. The $N$ ages in the grid are then associated with $\mathrm{N}$ single representative $[\mathrm{Fe} / \mathrm{H}]$ values, obtained as the mean metallicity value weighted by the KDE function at that particular age. The formal equation can be written as

$\overline{[\mathrm{Fe} / \mathrm{H}}_{\mathrm{age}_{i}}=\frac{\sum w_{i}[\mathrm{Fe} / \mathrm{H}]_{i}}{\sum w_{i}}$,

where the summations are performed over the $\mathrm{N}$ steps in the metallicity range, $[\mathrm{Fe} / \mathrm{H}]_{i}$ is the metallicity value at step $i$, and $w_{i}$ is the value of the $2 \mathrm{D} \mathrm{KDE}$ map for that fixed age and metallicity coordinates. The age ${ }_{i}$ subindex in Eq. (D.1) indicates that this mean metallicity was calculated for a fixed age value and, thus, represents a unique point in the AMR. A similar version of this method was employed in Noël et al. (2009, see Eq. (3)) to derive AMR estimates for three observed fields. We apply the above formula to all ages in the grid defined at the beginning of the process. The standard deviation for each $\overline{[\mathrm{Fe} / \mathrm{H}]}_{\mathrm{age}_{i}}$ value is calculated as

$\sigma_{\mathrm{age}_{i}}^{2}=\frac{\sum w_{i} \sum\left[w_{i}\left([\mathrm{Fe} / \mathrm{H}]_{i}-\overline{[\mathrm{Fe} / \mathrm{H}}_{\mathrm{age}_{i}}\right)^{2}\right]}{\left(\sum w_{i}\right)^{2}-\sum w_{i}^{2}}$,

where again all summations are applied over N, and the descriptions given for the parameters in Eq. (D.1) apply. At this point, this method already gives us an AMR function estimate, since every age step is mapped to a unique metallicity. The downsides are that the AMR is noisy owing to the very small step of 0.01 dex used and the associated errors are quite large. This latter effect arises because the weighted standard deviation, Eq. (D.2), is affected not only by errors in both measured parameters but also by the intrinsic dispersion in the metallicity values found for any given age. We therefore calculate the average $[\mathrm{Fe} / \mathrm{H}]$ for an age interval, rather than assigning a metallicity value to each age step in the grid. Dividing the age range into intervals requires a decision about the step width, much like when constructing a histogram, thereby bringing back the issue of binning. We have two advantages here: first, we use Knuth's algorithm (see Sect. 3.4) to obtain the optimal binning for our data; second, the final AMR function is very robust to changes in the binning method selected, so even the previous choice is not crucial in determining the shape of our AMR. Finally, the $\overline{[\mathrm{Fe} / \mathrm{H}}_{\mathrm{age}_{i}}$ values obtained for every age ${ }_{i}$ within a defined age interval are averaged. Errors are propagated through the standard formula disregarding covariant terms (Bevington \& Robinson 2003, Eq. (3.14)).

In Fig. D.1 we show the AMRs for both Clouds, which are generated using metallicity and age values taken from the literature articles, see Table 1 . Most clusters in these works are assigned fixed metallicities of $-0.7 \mathrm{dex}$ (SMC) and $-0.4 \mathrm{dex}$ (LMC), particularly for estimated ages below 1 Gyr. This explains the average difference of $\sim 0.2$ dex that can be appreciated, when compared to the ASteCA AMRs (Fig. 13). 

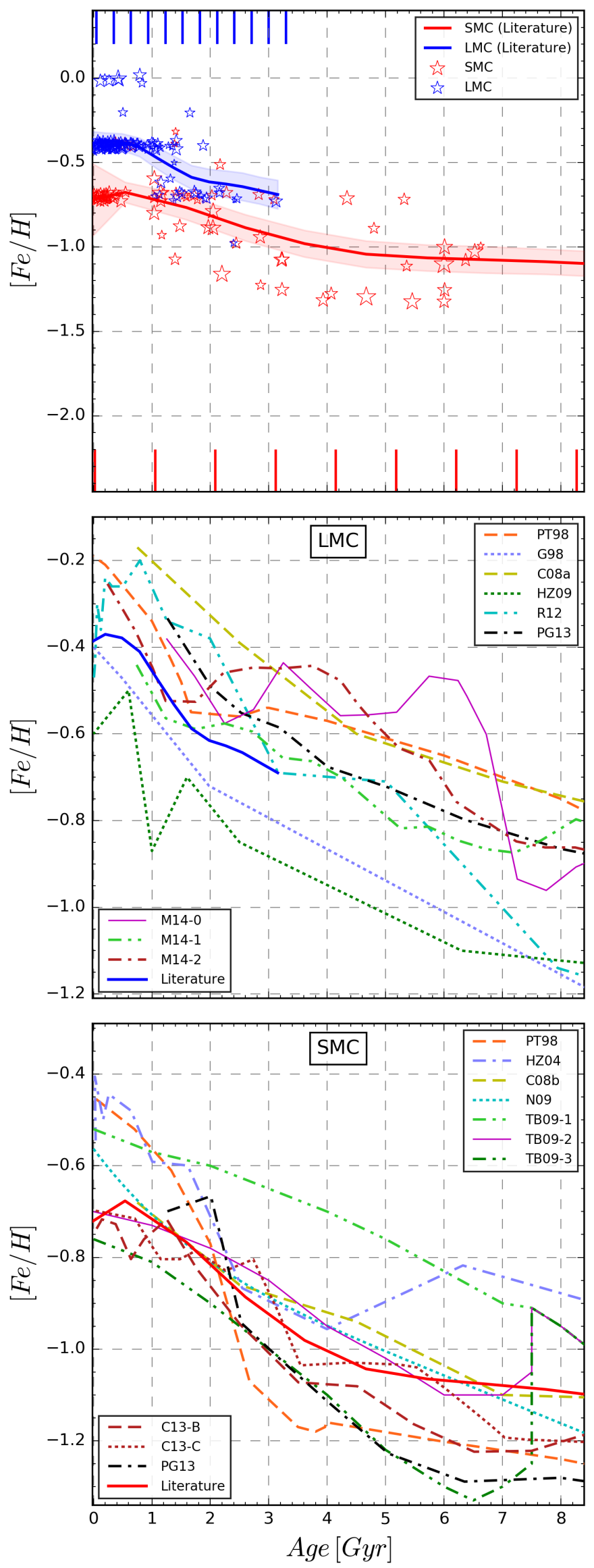

Fig. D.1. Age-metallicity relationships for our set of 239 clusters, using $\log ($ age $/ \mathrm{yr})$ and $[\mathrm{Fe} / \mathrm{H}]$ values taken from the literature. 